NBSIR 73-314

\title{
Study of Measurements of Antenna-Radiometer Parameters
}

Final Report on Phase I: Evaluation of Antenna Loss and Beam Efficiency Measurements and Radiometer Calibrations for the S.193 and S.194 Skylab Systems
A.C. Newell
P.F. Wacker
D.F. Wait
W.C. Daywitt
R.C. Baird

Electromagnetics Division

Institute for Basic Standards

National Bureau of Standards

Boulder, Colorado 80302

May 1973

Prepared for

NASA - Manned Spacecraft Center

Experiment Systems Division

Houston, Texas 



\section{Study of Measurements of Antenna-Radiometer Parameters}

Final Report on Phase 1: Evaluation of Antenna Loss and Beam Efficiency Measurements and Radiometer Calibrations for the S-193 and S-194 Skylab Systems

The following changes should be made in this report.

Page 6. The error estimates for $\mathrm{T}_{\mathrm{B}}$ given in the last three columns of Table 1.2.3.5 should be changed to:

$\begin{array}{llll}\mathrm{T}_{\mathrm{B}}=175 \mathrm{~K} & \pm 12.2 \mathrm{~K} & 11.9 \mathrm{~K} & 3.5 \mathrm{~K} \\ \mathrm{~T}_{\mathrm{B}}=350 \mathrm{~K} & \pm 37.2 \mathrm{~K} & 18.1 \mathrm{~K} & 6.6 \mathrm{~K}\end{array}$

Page 12. In equation (2-7), the second factor in the numerator should be $\left(1-S_{22} \Gamma_{\ell}\right)$ instead of $\left(1-S_{11} \Gamma_{\ell}\right)$.

Page 46. In the fourth line of Section 3.4, change \pm 14 to $\pm 42 \mathrm{~K}$ to read. \pm 12 to $\pm 49 \mathrm{~K}$.

Page 52. In equation (3-48), replace $T_{0}$ with $T_{0}^{\prime} \cdot$

The 5 th line from the bottom should read $81 \mathrm{~K}$ instead of $80 \mathrm{~K}$.

Page 53. The last line in the second paragraph should give 0.96 for the value of $M_{A}$ instead of 0.81 .

Page 54. The 9th line should read 372 Kelvins instead of 272 .

The value of $\mathrm{T}_{\mathrm{AH}}$ in the last line should be $372 \pm 2 \mathrm{~K}$ instead of $372.2 \pm 2 \mathrm{~K}$.

Page 57. Insert equation number $(3-56)$ to the right of the values given in lines 5 through 10 .

In the last paragraph,

$$
\begin{aligned}
& \left|\Gamma_{S W}\right|=0.025 \text { is printed twice, } \\
& \left|\Gamma_{F}\right|=0.17 \text { instead of } 0.017 \\
& R=1.03 \pm 0.026 \text { instead of } 1.03 \pm 0.027
\end{aligned}
$$



Page 58. Equation (3-58) should read

$$
\left|\mathrm{T}_{\text {AMP }}-\mathrm{T}^{\prime}{ }_{\mathrm{AMP}}\right| \cong 1.8 \mathrm{~K} \text {. }
$$

In the second line of the second paragraph, delete the $\%$ sign so that $a=0.86 \pm 0.16$.

Change the last line of the second paragraph to read "... about $0.16\left(\mathrm{~T}_{\mathrm{A}}-\mathrm{t}_{\mathrm{L}}\right) /(\alpha \mathrm{MAIN})^{2}$ and $0.009 \mathrm{~T}_{\mathrm{B}}$, respectively (refer to Table 3.4, page 46)".

In the third paragraph, replace $(3-56)$ with $(3-47)$.

Also, in the second line of the third paragraph, delete the phrase "listed in order of their importance".

Page 59. Replace the old page 59 with the new page 59 (attached).

Page 60. Replace the old Table 3.6 with the new Table 3.6 (attached). 
Table 3.5 Errors in Antenna Brightness Temperature For the Typical Conditions ${ }_{\mathrm{D}}=297 \mathrm{~K}, \mathrm{t}_{\mathrm{L}}=190 \mathrm{~K}, \alpha{ }^{\mathrm{MATN}}=0.84, \mathrm{~T}_{\mathrm{ANT}}=34 \mathrm{~K}, \alpha_{\mathrm{FA}} \simeq 0.8$.

\begin{tabular}{|c|c|c|c|}
\hline $\begin{array}{c}\text { Parameter } \\
\text { Causing Error }\end{array}$ & $\begin{array}{c}\mathrm{T}_{\mathrm{B}}=0 \quad \mathrm{~K} \\
\left(\mathrm{~T}_{\mathrm{A}}=41.7 \mathrm{~K}\right)\end{array}$ & $\begin{array}{c}\mathrm{T}_{\mathrm{B}}=175 \mathrm{~K} \\
\left(\mathrm{~T}_{\mathrm{A}}=184.4 \mathrm{~K}\right)\end{array}$ & $\begin{array}{c}T_{B}=350 \mathrm{~K} \\
\left(T_{A}=327.1 K\right)\end{array}$ \\
\hline Calibration & $\pm 12.3 \mathrm{~K}$ & $\pm 6.1 \mathrm{~K}$ & $\pm \quad 3.7 \mathrm{~K}$ \\
\hline$\alpha$ & 33.6 & 1.3 & 31.1 \\
\hline Beam Efficiency & 0.0 & 1.6 & 3.1 \\
\hline $\mathrm{R}$ & 6.6 & 2.9 & 0.8 \\
\hline $\mathrm{T}_{\mathrm{AMP}}$ & 2.2 & 2.2 & 2.2 \\
\hline Resolution & 1.3 & 1.3 & 1.3 \\
\hline Update & 0.7 & 0.7 & 0.7 \\
\hline Linearity & 0.4 & 0.4 & 0.4 \\
\hline Linear Sum & \pm 57.1 & \pm 16.5 & \pm 43.3 \\
\hline Quadratic Sum & \pm 36.5 & \pm 7.6 & \pm 31.6 \\
\hline $\begin{array}{l}\text { Quad. except } \\
\text { Cal. and TAMP }\end{array}$ & \pm 48.8 & \pm 12.2 & \pm 37.2 \\
\hline
\end{tabular}

$\pm 6.1 \mathrm{~K}$ in the process of transferring the external calibration to the internal reference generators, $\pm 2.9 \mathrm{~K}$ to account for the errors due to the mismatch between the antenna and the radiometer versus the standard and the radiometer, and $\pm 2.2 \mathrm{~K}$ because the effective input noise temperature of the amplifier changes when the antenna is replaced by the standard. Thus a $\pm 11.2 \mathrm{~K}$ error component in the $\pm 16.5 \mathrm{~K}$ resultant error is due to using a standard with an impedance different than the antenna's.

If the loss measurement errors were $\pm 0.02 \mathrm{~dB}$ (as claimed), then these values could be used to calculate the reference temperature $\mathrm{T}_{\mathrm{AH}}^{*}$ and $\mathrm{T}_{\mathrm{AC}}^{*}$ to within $2 \mathrm{~K}$ instead of \pm 3 and \pm 4.2 as given in (3-54) and (3-55). Further, (3-50) could be used instead of (3-49), to eliminate errors due to $R$ and $T_{A M P}$. Under these conditions, the errors shown in Table 3.6 would hold and the quadrature sum should be valid. In case the old loss measurements are not trusted, a variable impedance standard 

Table 3.6 Probable Attainable Errors in Antenna Brightness Temperature

\begin{tabular}{|c|c|c|c|c|c|c|}
\hline $\begin{array}{c}\text { Parameter } \\
\text { Causing Error }\end{array}$ & $\begin{array}{r}\mathrm{T}_{\mathrm{H}} \\
\mathrm{C} \\
\mathrm{A}\end{array}$ & $\begin{array}{l}=0 \\
41.7 \mathrm{~K}) \\
\end{array}$ & $\left(\mathrm{T}_{\mathrm{A}}^{\mathrm{T}}=\right.$ & $\begin{array}{l}=175 \mathrm{~K} \\
84.4 \mathrm{~K})\end{array}$ & $\begin{array}{r}\mathrm{T}_{\mathrm{H}} \\
\left(\mathrm{T}_{\mathrm{A}}=\mathrm{=}\right.\end{array}$ & $\begin{array}{l}=350 \mathrm{~K} \\
327.1 \mathrm{~K})\end{array}$ \\
\hline$\alpha$ & \pm & $6.3 \mathrm{~K}$ & \pm & $0.2 \mathrm{~K}$ & \pm & $5.8 \mathrm{~K}$ \\
\hline Beam Efficiency & & 0.0 & & 0.7 & & 1.4 \\
\hline Calibration & & 7.3 & & 3.1 & & 2.5 \\
\hline Resolution & & 1.3 & & 1.3 & & 1.3 \\
\hline Update & & 0.7 & & 0.7 & & 0.7 \\
\hline Linearity & & 0.4 & & 0.4 & & 0.4 \\
\hline Quadratic Sum & \pm & $9.8 \mathrm{~K}$ & \pm & $3.5 \mathrm{~K}$ & \pm & $6.6 \mathrm{~K}$ \\
\hline
\end{tabular}




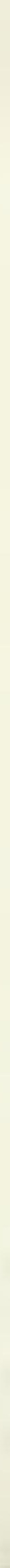


STUDY OF MEASUREMENTS OF ANTENNA-RADIOMETER PARAMETERS

Final Report on Phase I: Evaluation of Antenna Loss and

Beam Efficiency Measurements and Radiometeter Calibrations for the S-193 and S-194 Skylab Systems
A.C. Newell
P.F. Wacker
D.F. Wait
W.C. Daywitt
R.C. Baird

Electromagnetics Division

Institute for Basic Standards

National Bureau of Standards

Boulder, Colorado 80302

May 1973

Prepared for

NASA - Manned Spacecraft Center

Experiment Systems Division

Houston, Texas

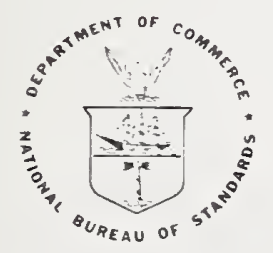

U.S. DEPARTMENT OF COMMERCE, Frederick B. Dent, Secretary National Bureau of Standards, Richard W. Roberts, Director 

$\underline{\text { Page }}$

ABSTRACT - - - - - -

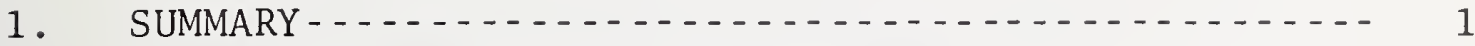

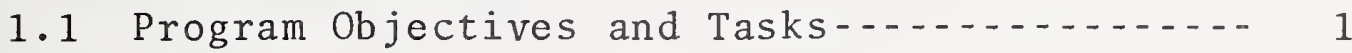

1.2 Summary of Phase I Results-............ 2

1.2.1 Description of Phase I Effort-.....-2 2

1.2.2 Summary of Section 2 - - Basic Concepts in Loss Measurements-........... 2

1.2.3 Summary of Section 3 -- Measurements on the S-194 System-............... 3

1.2.4 Summary of Section 4 -- Measurements on the S-193 System-............. 5

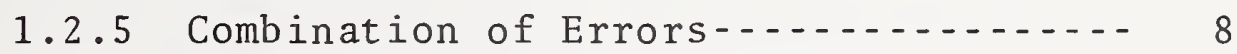

2. FUNDAMENTALS OF ANTENNA LOSS MEASUREMENTS-...-.-. 10

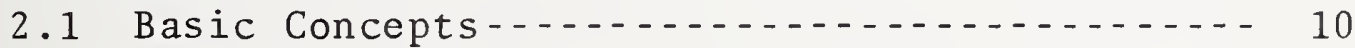

2.2 Measurement Methods -...................... 14

3. MEASUREMENTS ON THE S-194 SYSTEM-.................. 16

3.1 Loss Measurements on the S-194 Antenna-...- 16

3.1.1 Transmission Line Efficiency (Loss)

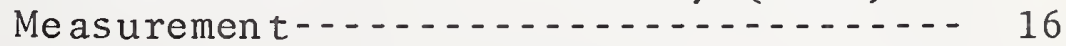

3.2 Gain Measurements on the S-194 Antenna--.-. 27

3.3 Antenna Pattern, Beam Efficiency, and

Directivity on the S-194 Antenna--....-. 33

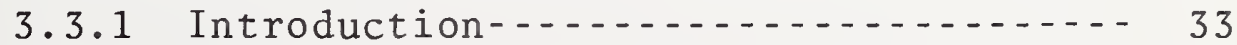

3.3.2 Properties of the Mode1 Mount-.....- 36

3.3.3 Symmetry, Polarization, and Scattering 41

3.3.4 Error Estimates................ 45

3.4 Calibration of the S-194 Radiometer-...... 46

3.4.1 Description of the S-194 Radiometer--- 47

3.4.2 The Accuracy of the S-194 Radiometer-- 52

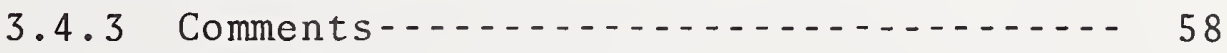

4. MEASUREMENTS ON THE S-193 SYSTEM-.............. 61

4.1 Loss Measurements on the S-193 Antenna---.-- 61

4.2 Gain Measurements on the S-193 Antenna---.-- 67 

$\underline{\text { Page }}$

4.3 Pattern Measurement, Beam Efficiency, and

Directivity of the S-193 Antenna-....... 72

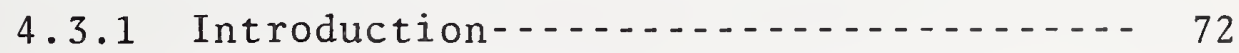

4.3.2 General Measurement Approach-.-..-. 74

4.3.3 Measurement Procedure used by the

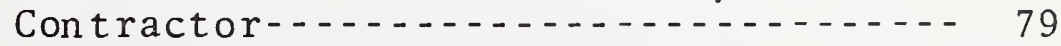

4.3.4 Beam Efficiency Calculations--..-.-. 93

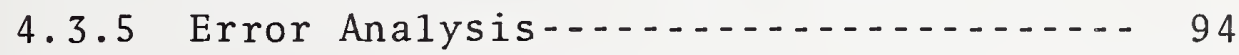

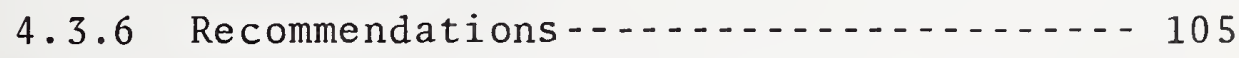

4.3.7 Comparison of Gain, Efficiency and

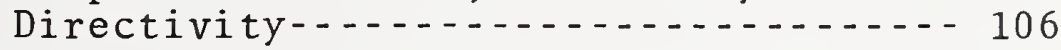

4.4 S-193 Radiometer Receiver Error Analysis---- 107

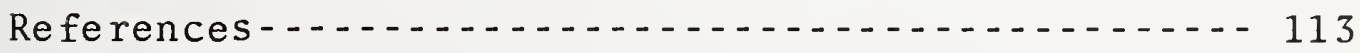


Final Report on Phase I: Evaluation of Antenna Loss and Beam Efficiency Measurements and Radiometer Calibrations for the S-193 and S-194 Skylab Systems

A.C. Newe11, P.F. Wacker, D.F. Wait, W.C. Daywitt and R.C. Baird

\section{Abstract}

The program represents an effort to determine the most appropriate techniques for measuring the critical parameters of the radiometer-antenna systems used in the NASA Earth Resources and Skylab programs. For this first phase, the contractors' measurements on the S-193 and S-194 Skylab systems were studied in detail. The measured quantities included antenna loss, antenna power gain, antenna patterns (which yield directivity and beam efficiency), and radiometric noise temperatures.

From the analysis of the contractors' data and auxiliary information, best values and their estimated errors were determined for each of the parameters. In addition, specific recommendations were made for easy upgrading of individual measurements, and the errors thus reduced were estimated. For some of the parameters, errors attainable with the current state-of-the-art were estimated.

In the measurements of antenna "loss," both contractors determined insertion loss rather than the ratio of output available power to input available power, required for radiometric determinations. For systems with significant reflections, these two quantities may be rather different, and the available power ratio should always be specified.

Antenna measurements made in an anechoic chamber showed surprisingly large wall reflections, suggesting that such chambers should be used for cross polarization measurements only with great caution.

Key Words: Antenna; antenna loss; beam efficiency; calibration and measurement; radiometer. 
Final Report on Phase I: Evaluation of Antenna Loss and

Beam Efficiency Measurements and Radiometer Calibrations for the S-193 and S-194 Skylab Systems

A.C. Newe11, P.F. Wacker, D.F. Wait, W.C. Daywitt and R.C. Baird

\section{SUMMARY}

\subsection{Program Objectives and Tasks}

The objectives of this program are to determine the most appropriate techniques for measuring the critical parameters of the radiometer antenna systems used in the NASA Earth Resources and Skylab programs. These parameters include antenna and radome losses, and antenna gain, pattern, and beam efficiency.

The program was divided into the following two phases. Phase I: A detailed study of measurements performed by contractors on the S-193 and S-194 Skylab systems. The purpose of this study was to determine the best values and associated uncertainties of the parameters measured by the contractors, and to indicate how these results could be readily upgraded. This report contains the results of this study.

Phase II: Determination of the best techniques for evaluating the aforementioned parameters. Significant improvement in the accuracies of some quantities can be achieved through relatively small efforts, as indicated in this report. Other quantities (particularly those associated with loss) will require additional study and research to determine the best approaches. These efforts will be discussed in future reports. 


\subsection{Summary of Phase I Results}

1.2.1 Description of Phase I Effort

For each system (S-193 and S-194), the contractors' determinations of the following quantities were analyzed:

- Antenna Loss

- Antenna power gain

- Antenna patterns (which yield directivity and beam efficiency)

- $\quad$ Radiometer noise temperature.

The input data we re obtained primarily from the contractors' test reports and from private conversations with the engineers involved. Insofar as possible, measurement procedures, methods of analysis, measured quantities, final calculated parameters, and estimates of errors were all evaluated. In cases where insufficient information concerning error sources was available from the contractor, errors were assigned on the basis of past experience by NBS staff members. An effort was made to obtain all pertinent data available so that the conclusions would accurately represent the facts.

The detailed findings of this study are contained in sections 2, 3, and 4, but the main results and recommendations of each section are summarized here as an aid in the use of the report.

1.2.2 Summary of Section 2 - Basic Concepts in Loss Measurements

It became apparent early in the study that the term "loss" as applied to antennas and transmission line components was being used rather loosely, and, as a result, the correct quantity was not being measured. The correct loss parameter for evaluating radiometer system performance is given in 2 . The errors resulting from use of the wrong loss parameter are most significant when the test and/or measurement systems have significant impedance mismatches. This was particularly evident in the S-194 analysis as is indicated in the next section. 
The definition of the correct loss parameter (eqs. 2.2 and 2.3) should be included in future specifications of radiometer systems. This would help ensure measurement of the correct quantity and use of the best measurement techniques by all contractors.

The advantages and disadvantages of the two methods of determining antenna loss considered by the contractors are presented. One technique requires gain and complete pattern measurements, from which the loss is computed as the ratio of gain to directivity. The second method involves measuring the loss in all readily accessible components of the feed system and calculating or estimating the losses in those parts of the antenna system that are not amenable to measurement.

\subsubsection{Summary of Section 3-- Measurements on the S-194 System}

\subsubsection{Antenna Loss Measurements}

The contractor measured the insertion loss of the main power divider and assumed the 8 secondary dividers to be identical to the main divider. He neglected the losses in the radiating elements and the radome and reported the antenna loss to be $0.284 \mathrm{~dB}$. Due to significant mismatch problems and use of the wrong loss parameter, there is a large error in this reported value. Even assuming the dividers are identical and the radiating elements are lossless, the correct loss is estimated to be $0.68 \mathrm{~dB}+0.68 \mathrm{~dB}$.

It would be difficult, if not wholly impractical, to obtain sufficient information on this system to completely resolve the mismatch problem. The losses in the radiating elements and radome are also difficult to determine. It is therefore recommended that either measurements of gain and directivity or radiometric measurements be used to obtain a 
more accurate antenna loss value. Using the gain and directivity values computed by the NBS (see Section 1.2.3.5) from the contractor's data, a value of $0.37 \mathrm{~dB}+0.5 \mathrm{~dB}$ was obtained for the antenna loss.

\subsubsection{Antenna Gain Measurements}

The major sources of error in the gain determination were an uncalibrated gain horn, multipath signals within the anechoic chamber, and impedance mismatches. These factors result in an estimated error of $\pm 0.41 \mathrm{~dB}$ in the gain of the S-194 antenna. This error could be reduced to about $\pm 0.20 \mathrm{~dB}$ by calibrating the gain horn together with further analysis of mismatch and multipath interference. New techniques in use at NBS could be used to calibrate the S-194 antenna directly within about $\pm 0.10 \mathrm{~dB}$.

\subsubsection{Antenna Pattern, Beam Efficiency, and Directivity}

Scattering within the chamber where the pattern measurements were performed was a problem. This scattered energy caused significant errors in the principal polarization patterns, but the effect was partially removed by a renormalization of the patterns. The measured cross polarization patterns of the S-194 antenna are so variable (due to the scattered energy) that they are of little, if any, value. However, even if the recorded patterns did accurately represent the situation, the total cross-polarized power is negligible with respect to the power in the principal polarization and, therefore, has negligible effect on the beam efficiency and directivity. The total estimated errors due to all sources are $\pm 0.14 \mathrm{~dB}$ in directivity and $0.9 \%$ in beam efficiency.

The fact that the received power varied by $2 \mathrm{~dB}$ as the antenna pair rotated with respect to the chamber indicates 
that more care should be exercised in the future in using an anechoic chamber for complete pattern measurements of this type.

\subsubsection{Radiometer System Evaluation}

The contractor estimates that the S-194 radiometer can measure brightness temperature at $175 \mathrm{~K}$ and $350 \mathrm{~K}$ with probable errors of $\pm 2.1 \mathrm{~K}$ to $\pm 4.2 \mathrm{~K}$ respectively. Our analysis indicates that the errors are in the range $\pm 14 \mathrm{~K}$ to $\pm 22 \mathrm{~K}$ for the same temperatures. The two largest sources of error are the uncertainties in the antenna loss and in the noise standards used to calibrate the radiometer. (See table 3.5 page 59 for a complete listing of the sources of error in determining brightness temperature.) There is a significant mismatch problem because the impedances of the antenna and standards are not the same. Alternative approaches are recommended which could be used for this and other systems to reduce the calibration error. If the improved calibration is used, and the uncertainty in antenna loss is lowered to about $0.13 \mathrm{~dB}$ by reducing the error in antenna gain as discussed in 1.2 .3 .2 , the above errors would be reduced to $\pm 3.1 \mathrm{~K}$ and $\pm 4.7 \mathrm{~K}$.

1.2.4 Summary of Section 4-- Measurements on the S-193 System

\subsubsection{Antenna Loss Measurements}

The contractor determined the loss of the S-193 antenna by a combination of measurements and calculations. The losses of the rectangular waveguide section and the mode transducer in the feed were measured directly. As with the S-194, an incorrect definition of loss was used but, since mismatch errors were small, the resulting error was on the order of a few hundredths of $a d B$. The loss in the remainder of the feed (circular waveguide, tapered dielectric guide, reflectors, etc.) 


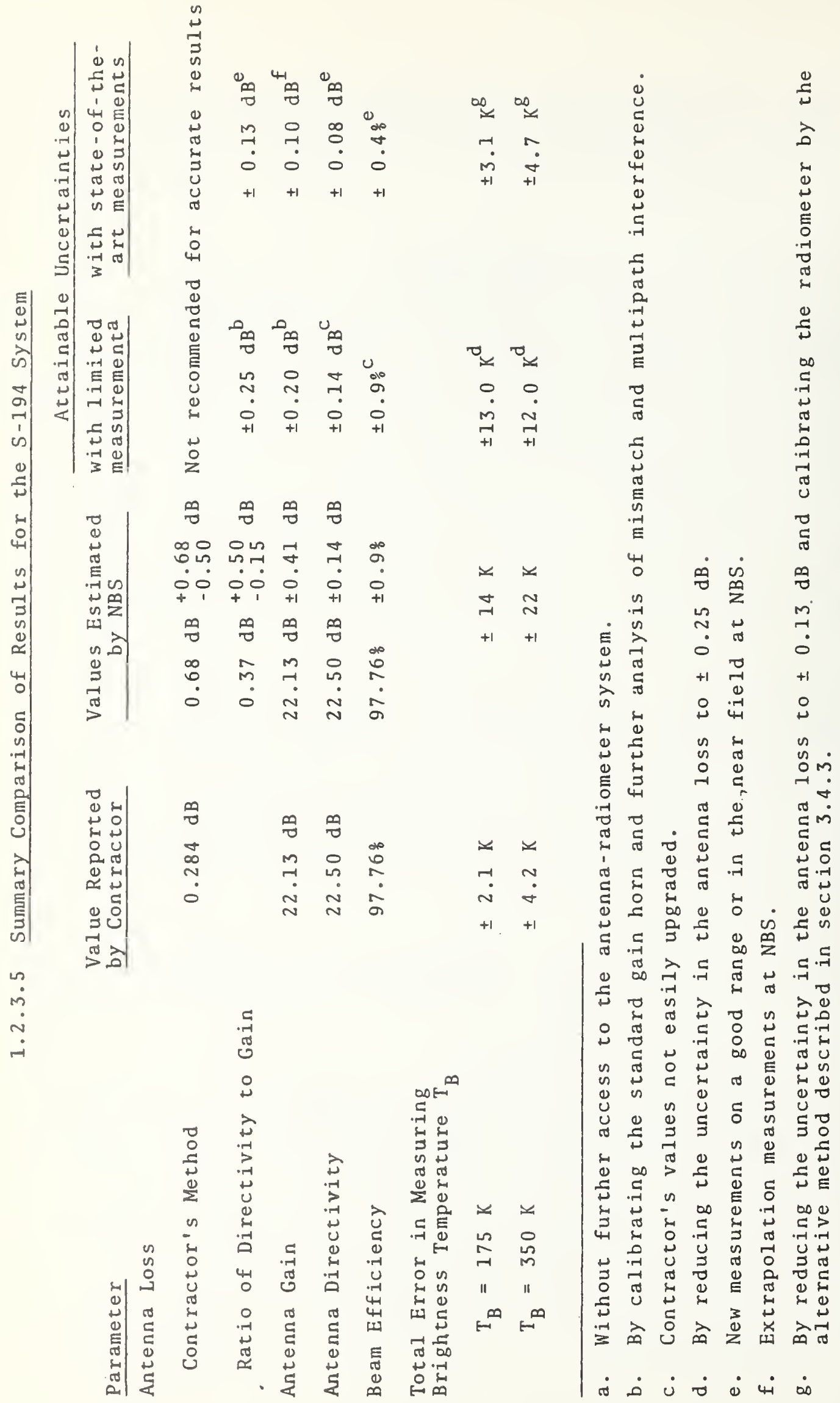


was determined from theoretical calculations. While this approach is probably valid for the fairly simple geometry involved, it does pose some problems. There could be flaws in the construction of the dielectric feed which would cause higher loss and, if no actual loss measurements are performed, these flaws could go undetected. Also, the approximations and assumed parameters used in the theoretical calculations may not be completely valid. It would be desirable to actually measure the loss of the total assembly and avoid these questions.

Gain and directivity computed from pattern measurements could be used to obtain the antenna loss, but the existing pattern measurements for this system are not sufficiently accurate.

\subsubsection{Antenna Gain Measurements}

The major errors in this measurement were attributed to an uncalibrated gain standard and the use of an uncalibrated receiver for measuring the received power ratios. The resultant error of $\pm 0.37 \mathrm{~dB}$ could be reduced to about $\pm 0.15 \mathrm{~dB}$ by calibration of the gain standard and receiver, or the S-193 antenna could be calibrated directly to within about $0.1 \mathrm{~dB}$ at the National Bureau of Standards.

\subsubsection{Antenna Pattern, Beam Efficiency, and Directivity}

Complete pattern measurements were not performed on the S-193 antenna. Patterns were measured in two of four "quadrants" which comprise the total sphere, and symmetry was assumed to obtain the patterns in the other two "quadrants." Analog patterns were recorded for part of the remaining region, but these data were not used in the pattern integration. The assumed symmetry is clearly not justified, as partial patterns for the other two quadrants illustrate. Therefore, the lack of complete data has caused a signficant error in the calculated beam efficiency and directivity. 
An additional error of about $6 \%$ in the beam efficiency has been introduced through use of an incorrect numerical integration procedure. This error could be eliminated if the integration was redone correctly. At present there is an estimated error of about $+3 \pm 2.8 \%$ in the beam efficiency, which could be reduced to about $\pm 2.0 \%$ by correcting the integration and making complete pattern measurements. The remaining error is due to a combination of receiver response error, range multipath effects, and uncertainties in the main beam boundary.

\subsubsection{Radiometer Receiver Evaluation}

Although certain simplifying assumptions were made by the contractor in the radiometer receiver calibration, these caused little error. The total probable error in the measured brightness temperature due to the receiver is estimated to be $\pm 1.5 \mathrm{~K}$ for a $50 \mathrm{~K}$ incident temperature and $\pm 0.3 \mathrm{~K}$ for a $350 \mathrm{~K}$ incident temperature.

The errors due to uncertainty in antenna loss and beam efficiency must be added to these numbers to obtain the total errors which are $\pm 9.5 \mathrm{~K}$ for a $50 \mathrm{~K}$ incident temperature and $\pm 12.3 \mathrm{~K}$ for a $350 \mathrm{~K}$ incident temperature.

\subsubsection{Combination of Errors}

For systematic errors, worst case estimates were generally used, and for random error, $2 \sigma$ values. In cases where it was apparent that the individual sources of error were independent with roughly normal distributions, the errors were combined by a quadrature summation. This was true in most cases, and the resulting combination is equivalent to a $97 \%$ confidence level. Where the errors did not satisfy the above conditions, a direct sum was used. 


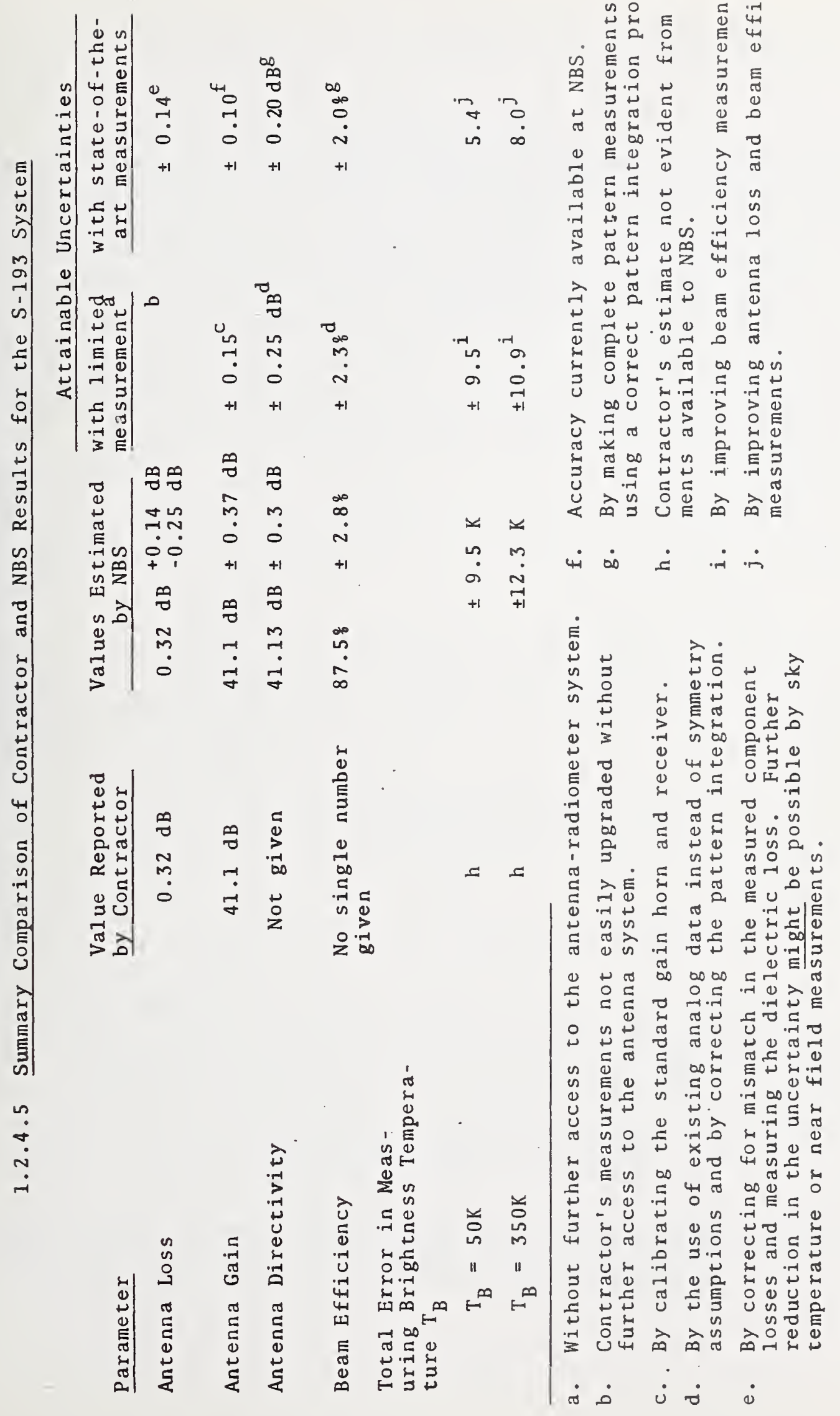


2. FUNDAMENTALS OF ANTENNA LOSS MEASUREMENTS

\subsection{Basic Concepts}

Since a major concern of this report is to evaluate loss measurements on a noise measuring system, it seems appropriate to define precisely which "loss" should actually be measured, how it is defined and influenced by other system parameters, and how it may be measured or calculated. We will consider first a passive, linear two-port at a temperature $\mathrm{T}$ with a noise generator at temperature $\mathrm{T}_{\mathrm{g}}$ connected to one port and a load connected to the other port (see Fig. 2-1).

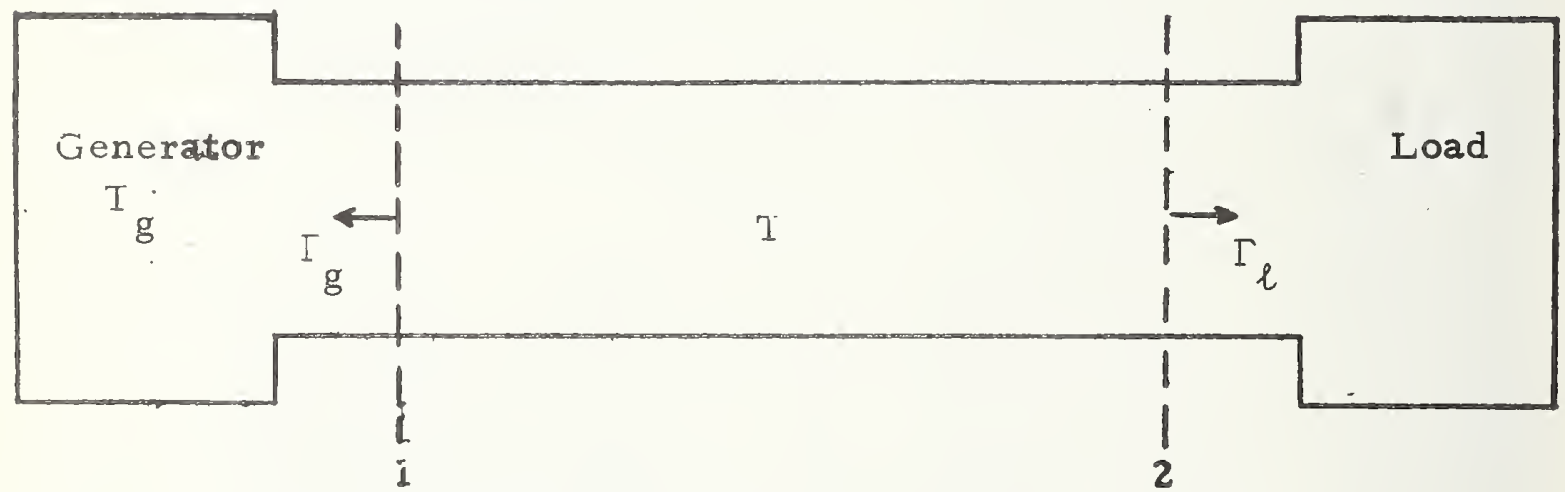

Figure 2-1. Two-port with generator and load.

The power per unit bandwidth available to the load is given by [1],

$$
P_{a l}=k\left[\alpha_{21} T_{g}+\left(1-\alpha_{21}\right) \mathrm{T}\right]
$$

(In the remaining equations in this section, the power referred to will be understood to mean power per unit bandwidth.) The constant $\alpha_{21}$ is defined as the ratio of available powers,

$$
\alpha_{21}=\frac{\text { available power at port } 2}{\text { available power at port } 1} \text {, }
$$

and is usually referred to as the available power ratio. This quantity, $\alpha_{21}$, determines the reduction in available power due to 
internal dissipation and, as shown in (2-1), determines the amount of internal noise generated. Therefore $10 \mathrm{log} 1 / \alpha_{21}$ is the "loss" parameter which is required.

In terms of scattering parameters of the two port and the termination reflection coefficient, $\alpha_{21}$ is given by [2]

$$
\alpha_{21}=\frac{\left|S_{21}\right|^{2}\left(1-\left|\Gamma_{g}\right|^{2}\right)}{\left|1-S_{11} \Gamma_{g}\right|^{2}-\left|\left(S_{12} S_{21}-S_{11} S_{22}\right) \Gamma_{g}+S_{22}\right|^{2}} \text {. }
$$

From (2-3) it is apparent that $\alpha_{21}$ is a function of both the $S$ parameters of the two port and the source reflection coefficient, including mismatch effects as well as $\mathrm{S}_{21}$. This means that $\alpha_{21}$ must be measured with the proper generator impedance in place, or corrections made for the difference between generator impedances of the measuring and actual system.

If the two port is reciprocal, then it can be shown [2] that $\alpha_{21}$ is equal to $n_{12}$, where $n_{12}$ is the two port efficiency for power flow from 2 to 1 , i.e., in the reverse direction. The efficiency is defined as

$$
n_{12}=\frac{\text { Net delivered power at port } 1}{\text { Net delivered power at port } 2} \text {. }
$$

Therefore, for a reciprocal device, $\alpha_{21}$ may be measured directly, calculated from the scattering parameters, or determined by measuring $n_{12}$.

Since there are various other "loss" parameters of a two port, we will give below the expressions for these in terms of the scattering parameters and reflection coefficients of generator and load. A more complete discussion of these may be found elsewhere [3].

$$
\text { Insertion loss }=10 \log \frac{\left|\left(1-\mathrm{S}_{11} \Gamma_{g}\right)\left(1-\mathrm{S}_{22} \Gamma_{\ell}\right)-\mathrm{S}_{12} \mathrm{~S}_{21} \Gamma_{\mathrm{g}} \Gamma_{\ell}\right|^{2}}{\left|\mathrm{~S}_{21}\right|^{2}\left|1-\Gamma_{g} \Gamma_{\ell}\right|^{2}}
$$




$$
\text { Attenuation }=10 \log \frac{1}{\left|\mathrm{~S}_{12}\right|^{2}}
$$

Transducer $10 \mathrm{ss}=10 \log \frac{\left|\left(1-\mathrm{S}_{11} \Gamma_{\mathrm{g}}\right)\left(1-\mathrm{S}_{11} \Gamma_{\ell}\right)-\mathrm{S}_{12} \mathrm{~S}_{21} \Gamma_{\mathrm{g}} \Gamma_{\ell}\right|^{2}}{\left|\mathrm{~S}_{21}\right|^{2}\left(1-\left|\Gamma_{\mathrm{g}}\right|^{2}\right)\left(1-\left|\Gamma_{\ell}\right|^{2}\right)}$

If one of the above quantities is measured rather than $\alpha$ or $n$ and used in equation (2-1) to compute noise power characteristics, a significant error is likely to occur. For instance, take the case where a tuned reflectionless measurement system is used to measure the insertion loss of a two port section of transmission line. Since the measurement system is tuned, $\Gamma_{g}$ and $\Gamma_{\ell}$ are zero, and it is actually the attenuation which is being measured. If the transmission 1 ine is now placed in the circuit where it is actually going to be used, $\Gamma_{g}$ and $\Gamma_{\ell}$ will probably not be zero, and the efficiency and insertion loss of the transmission line will not be equal to the attenuation. The range of values that $n$ can have for various input, output, and generator reflection coefficients is shown in the table below.

Table 2.1 Possible Combinations of Attenuation and Efficiency

\begin{tabular}{|c|c|c|c|c|c|}
\hline$S_{11} \mid$ & $\left|S_{22}\right|$ & $\left|\Gamma_{\mathrm{g}}\right|$ & $\begin{array}{l}\text { Measured } \\
\text { Attenuation }(\mathrm{d} B)\end{array}$ & $\operatorname{Max}^{n}{ }^{i 1}$ & $\mathrm{~dB}$ \\
\hline$\cdot$ & $\begin{array}{l}.1 \\
.1 \\
.1 \\
.2\end{array}$ & $\begin{array}{l}.1 \\
.1 \\
.1 \\
.2\end{array}$ & $\begin{array}{l}-0.2 \\
-0.5 \\
-1.0 \\
-0.5\end{array}$ & $\begin{array}{l}-0.129 \\
-0.381 \\
-0.813 \\
-0.225\end{array}$ & $\begin{array}{l}-0.189 \\
-0.548 \\
-1.128 \\
-0.469\end{array}$ \\
\hline
\end{tabular}

For a multiport device the noise power relations are similar to those for a two port, but an a must be defined for each pair of ports. Consider a multiport at a temperature $\mathrm{T}$ with independent generators on each input port at temperature $\mathrm{T}_{j}$. The total available power at port i is given by

$$
P_{a i}=k\left[\sum_{j \neq i} \alpha_{i j} T_{j}+\left(1-\sum_{j \neq i} \alpha_{i j}\right) \mathrm{T}\right] \text {. }
$$


Once again, each $\alpha_{i j}$ is the ratio of available power out of port $i$ to the available power from the generator on port $j$, when only the generator $j$ has non-zero amplitude. Expressions are available [1] which give each $\alpha_{i j}$ in terms of the scattering parameters of the multiport and the generator reflection coefficients and, again, each a must be measured or calculated with proper consideration for the effects of generator impedance and interaction between ports.

The extension from a multiport to an antenna is fairly natura1. Consider a continuous brightness temperature distribution for each of two orthogonal polarizations being received by the receiving cross section function of the antenna, where each direction for each polarization corresponds to a "port." The total available output power is given by

$$
\mathrm{P}_{\mathrm{a}}=\mathrm{k}\left[\int \frac{\underline{\mathrm{T}}_{\mathrm{B}}(\theta, \phi) \cdot \underline{\mathrm{A}(\theta, \phi)}}{\lambda^{2}} \mathrm{~d} \Omega+(1-\alpha) \mathrm{T}_{\mathrm{a}}\right] \text {, }
$$

where $\underline{T}_{B}(\theta, \phi)$ is the brightness temperature, $\underline{A}(\theta, \phi)$ is the antenna receiving cross section, $\mathrm{T}_{\mathrm{a}}$ is the antenna temperature, and a the factor which describes the noise generating character of the antenna. Both $\underline{T}_{B}$ and ${ }_{-}$are two-vectors with the components corresponding to each of two orthogonal polarizations. By analogy to the multi-port case we can identify the $\alpha_{i j}$ 's for each port (direction) with the factor

$$
\alpha_{i j}=\frac{A(\theta, \phi)}{\lambda^{2}} d \Omega \text {, }
$$

and the total $\alpha$ as

$$
\alpha=\int \frac{A(\theta, \phi)}{\lambda^{2}} \mathrm{~d} \Omega \text {. }
$$

The expression for a may also be derived by considering the antenna and its load in thermal equilibrium with an incident uniform brightness temperature $T$. This derivation will lead to the same expression as $(2-11)$. 
If the antenna is reciprocal, then (2-11) becomes

$$
\alpha=\frac{G}{D}=\eta
$$

where $G$ and $D$ are, respectively, the gain and directivity in a given direction and $n$ is the antenna efficiency. In this case, $n$ may be determined from measurements on the antenna in either a transmitting or receiving mode. If the antenna is non-reciprocal, then the antenna must be used in the receiving mode when measuring $\alpha$.

\subsection{Measurement Methods}

Both vendors have sufficient data to determine $n$ by two different and independent techniques. The first method is to measure G, calculate D from the pattern data, and compute the ratio directly. This method has a number of advantages, especially when the pattern data are also necessary to compute beam efficiency. Some of these advantages are:

1. This approach will account for all lossy elements and their interactions without further calculation. This advantage is especially significant where there may be many elements (such as the power dividers and radiators in the S-194) or where the phase of the reflection coefficient is unknown.

2. If the gain and pattern need to be determined for other purposes, the efficiency may be obtained without additional measurements.

The disadvantages of this method are that it requires complete pattern data and computer processing to determine D. It also requires a good range and accurate standards to determine both gain and directivity. 
The second method involves a conceptual decomposition of the antenna system into segments. The loss associated with each segment is then either measured or computed. The advantages of this approach are that it requires fairly simple laboratory measurements and no antenna range is required. The loss in individual components may be determined with this method, which is an advantage in the design phase.

Some of the disadvantages of this approach are:

1. It neglects the loss in some parts of the antenna, and therefore is really only a lower bound to the total loss.

2. The efficiency of a given segment, which is defined as the ratio of the net power delivered to the load to the net input power, is a function of the scattering parameters of the segment and the load impedance. Since the load impedance of the measuring circuit may not be the same as the load which the line sees when used with the antenna, the correct efficiency is probably not measured.

3. When the system is divided into a number of cascaded elements, mismatch effects occur at each junction, and the measurements and/or calculations should include these effects.

It is apparent that a significant error occurred for at least one of the systems when this second approach was used to determine antenna efficiency. The reason for the error is that the insertion loss rather than the efficiency was measured, and there is a significant difference between the two for these systems. This will be more apparent as the measurements are examined in detail. 


\subsection{Loss Measurements on the S-194}

The errors in both types of measurement will be analyzed here using the data which the vendor obtained or which can be easily obtained.

\subsubsection{Transmission Line Efficiency (Loss) Measurement}

In this approach, the main 8 -way power dividers and the eight secondary 8 -way power dividers are assumed to be the only lossy part of the antenna. The total efficiency of the 64way power divider, resulting from combining the main and secondary dividers, is then taken to be the antenna efficiency.

Referring to the schematic shown in figure $3-1$, the following notation and definitions will be used.

$\mathrm{P}_{0}=$ Net power delivered to the main power divider when the output ports are terminated with actual loads.

$P_{i}(i=1$ to 8$)=$ Net power delivered to the input of each secondary power divider with actual loads in place. The actual loads are the loads which will be in place when the antenna is in use.

$Q_{i j}(i=1$ to $8 ; j=1$ to 8$)=$ Net power delivered to each radiating element.

$\mathrm{Q}_{0}=$ Total power delivered to the array.

$\eta_{m}=$ Efficiency of main divider.

$\eta_{i}=$ Efficiency of each the ith divider.

$n_{\mathrm{T}}=$ Total efficiency of combination 64-way divider. 


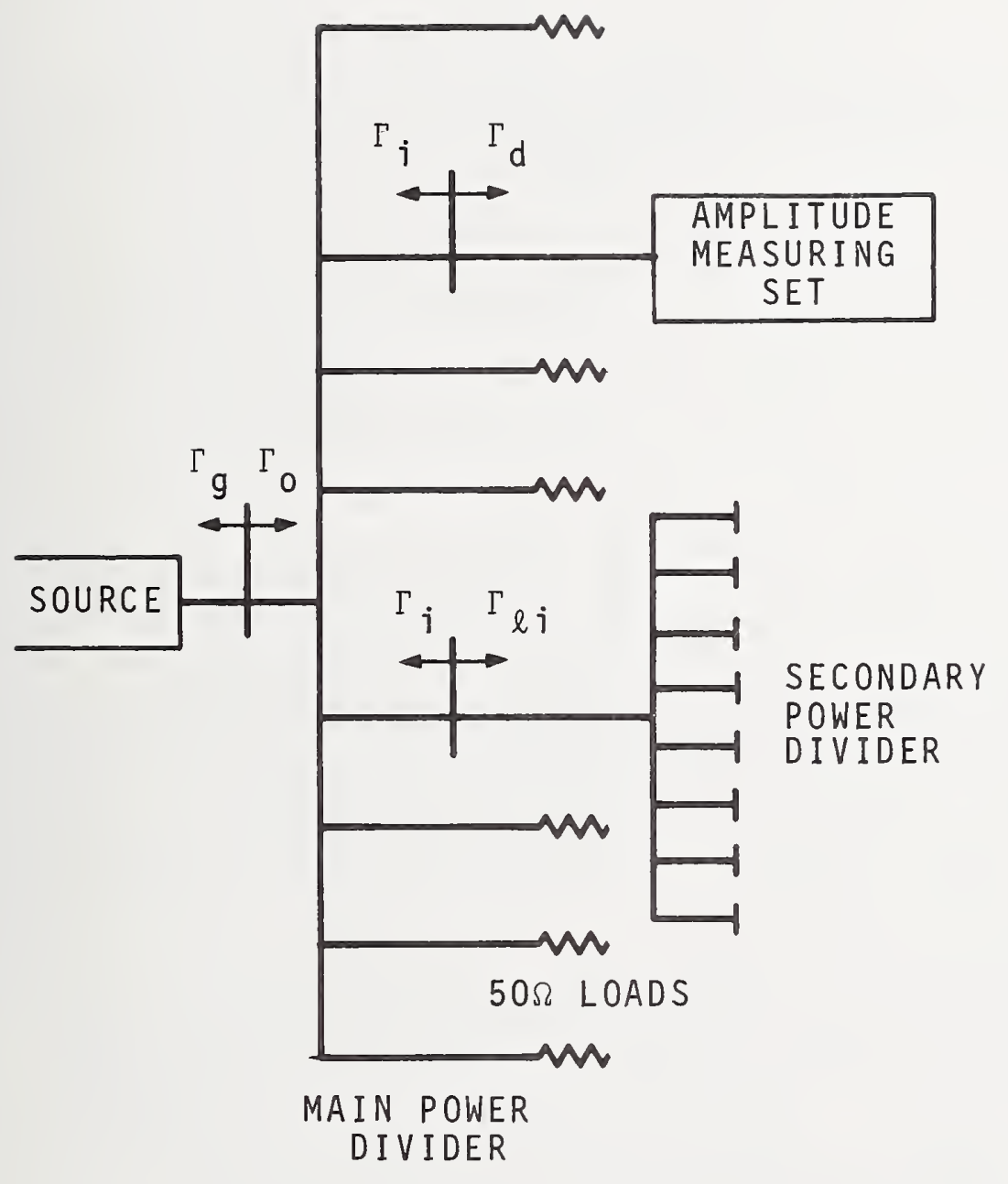

Figure 3-1. S-194 Power Divider 
We can write the following expressions for the efficiencies:

$$
\eta_{m}=\frac{\sum_{i=1}^{8} P_{i}}{P_{0}}, \eta_{i}=\frac{\sum_{j=1}^{8} Q_{i j}}{P_{i}}
$$

and

$$
\eta_{T}=\frac{Q_{0}}{P_{0}}=\frac{\eta_{m} \sum_{i=1}^{8} \eta_{i} P_{i}}{\sum_{i=1} P_{i}} .
$$

At this point, the contractor has assumed that the efficiencies of each of the eight secondary dividers are identical with the efficiency of the main divider. With this assumption eq. (3-2) becomes,

$$
n_{T}=\eta_{m}^{2}
$$

The total efficiency is then determined by measuring the efficiency of the main divider and squaring the results. The efficiency of the main divider was measured by the following steps:

1. All of the outputs of the main divider were terminated with low reflection loads.

2. The source was disconnected from the input to the divider and connected to the detector of an amplitude measuring circuit. This unit consisted of a superheterodyne mixer with a precision attenuator in the IF line. With the detector connected to the source, the attenuator was set on a convenient reading and the output meter reading noted.

3. The divider was reconnected to the source and the detector connected to one of the output ports. The attenuator was reset to reproduce the original meter reading and the change in attenuator setting recorded. 
This step was repeated for each of the other output ports to determine the insertion loss, $\mathrm{L}_{i}$, for each port.

Referring again to figure 3-1, we will use the following notation:

$\Gamma_{g}=$ Reflection coefficient of source generator.

$\Gamma_{d}=$ Reflection coefficient of detector.

$\Gamma_{0}=$ Reflection coefficient at the input to the main power divider.

$\Gamma_{i}(i=1$ to 8$)=$ Reflection coefficient of ith output port of divider, which is assumed to be the same for the measurement case and in actual use.

$\Gamma_{l i}(i=1$ to 8$)=$ Reflection coefficient of the load which the ith output port sees in actual use. This load consists of the ith secondary divider and the attached radiating elements.

$D_{0}=$ Power delivered to the detector when connected to the source.

$D_{i}(i=1$ to 8$)=$ Power delivered to detector when connected to ith output.

$\mathrm{b}_{\mathrm{g}}=$ Wave amplitude which the source would deliver to a matched (reflectionless) load.

$b_{g i}=$ Wave amplitude which the ith output would deliver to a matched (reflectionless) load with the remaining ports terminated in the actual loads and the input connected 
to the source. This is also assumed to be the same for the measurement case and actual use.

$z_{0}=$ Characteristic impedance of transmission 1 ine.

The measured ratio is,

$$
L_{i}=10 \log \left(\frac{D_{i}}{D_{0}}\right), \quad \frac{D_{i}}{D_{0}}=\log ^{-1}\left(\frac{L_{i}}{10}\right)=R_{i}^{\prime},
$$

and the computed apparent efficiency is then

$$
n_{m}^{\prime}=\sum_{i=1}^{8} R_{i}^{\prime}
$$

This is not the true efficiency because of the mismatch errors involved in the measurement. The magnitude of this mismatch error is determined as follows. In terms of the wave amplitudes and reflection coefficients, the delivered powers are

$$
\begin{aligned}
& \mathrm{D}_{0}=\frac{\left|\mathrm{b}_{\mathrm{g}}\right|^{2}}{z_{0}} \frac{1-\left|\Gamma_{d}\right|^{2}}{\left|1-\Gamma_{d} \Gamma_{g}\right|^{2}}, \\
& \mathrm{D}_{i}=\frac{\left|\mathrm{b}_{g i}\right|^{2}}{z_{0}} \frac{1-\left|\Gamma_{d}\right|^{2}}{\left|1-\Gamma_{d} \Gamma_{i}\right|^{2}}, \\
& \mathrm{p}_{0}=\frac{\left|\mathrm{b}_{g}\right|^{2}}{z_{0}} \frac{1-\left|\Gamma_{0}\right|^{2}}{\left|1-\Gamma_{0} \Gamma_{g}\right|^{2}}, \\
& \mathrm{p}_{i}=\frac{\left|b_{g i}\right|^{2}}{z_{0}} \frac{1-\left|\Gamma_{\ell i}\right|^{2}}{\left|1-\Gamma_{i} \Gamma_{\ell i}\right|^{2}} .
\end{aligned}
$$

If we $1 \mathrm{et}$

$$
R_{i}=\frac{P_{i}}{P_{0}}
$$


then

$$
R_{i}=\frac{\left|1-\Gamma_{0} \Gamma_{g}\right|^{2}}{\left|1-\Gamma_{d} \Gamma_{g}\right|^{2}\left(1-\left|\Gamma_{0}\right|^{2}\right)} \frac{\left(1-\left|\Gamma_{\ell i}\right|^{2}\right)\left|1-\Gamma_{d} \Gamma_{i}\right|^{2}}{\left|1-\Gamma_{\ell i} \Gamma_{i}\right|^{2}} R_{i}^{\prime}
$$

or

$$
R_{i}=M_{i} R_{i}^{\prime},
$$

where

$$
M_{i}=\frac{\left(1-\left|\Gamma_{\ell i}\right|^{2}\right)\left|1-\Gamma_{0} \Gamma_{g}\right|^{2}\left|1-\Gamma_{d} \Gamma_{i}\right|^{2}}{\left(1-\left|\Gamma_{0}\right|^{2}\right)\left|1-\Gamma_{d} \Gamma_{g}\right|^{2}\left|1-\Gamma_{\ell i} \Gamma_{i}\right|^{2}}
$$

$M_{i}$ is a mismatch correction in each ratio measurement. If we assume that $\Gamma_{i}$ and $\Gamma_{\ell i}$ are independent of the output port $i$ of the main divider, then we have

$$
n_{m}=\Sigma R_{i}=M_{m} \sum R_{i}^{\prime}=M_{m} n_{m}^{\prime}
$$

where $\eta_{m}^{\prime}$ is the apparent efficiency which was determined from the contractor's measurements, and $M_{m}$ denotes the mismatch correction for the main divider. The contractor's measurements on the power divider indicate that the assumption of identical $\Gamma_{i}$ 's and $\Gamma_{\ell i}$ 's is only approximately true, but is justified in estimating limits of error.

We will assume that the same conditions are true for the secondary dividers. That is, the output impedances and load impedances are the same for al1 64 ports. No data is available or obtainable to support this assumption, but it is probably justified because of the similarity of the dividers and the radiating elements. Data are not obtainable because there actually is no connector between the output of the secondary dividers and the input to the radiating elements. The dipoles are a continuation of the divider stripline and cannot be disconnected to make reflection coefficient measurements. It will be assumed that output impedances are the 
same as for the main divider, and that the load reflection coefficients are somewhat higher than those of the main divider.

It will also be assumed that the apparent efficiency of the secondary dividers is the same as the apparent efficiency of the main divider. This essentially means that the transmission coefficient in all dividers is the same. Again, this is probably justified on the basis of construction and is required for this measurement approach since direct loss measurements cannot be performed on the secondary dividers.

With the foregoing assumptions, the total efficiency is then

$$
n_{T}=M_{m} M_{s} n_{m}^{\prime 2}
$$

where $M_{S}$ is the mismatch correction for the secondary dividers. The error in determining $n_{\mathrm{T}}$ will then be due to the errors in each mismatch correction and $\eta_{m}$. These will now be discussed separately.

Errors in Measuring $n_{m}^{\prime}$

The sources of error in this measurement are as follows:

1. The accuracy of equipment used to measure $R_{i}^{\prime}$. It can be shown that a systematic error of $\Delta \mathrm{L}_{i} \mathrm{~dB}$ in each of the measured ratios will cause $10 \log n_{m}^{1}$ to be in error by the same amount, and therefore 10 log $\eta_{\mathrm{T}}^{\prime}$ will be in error by twice that amount. The precision attenuator used to measure the ratios is probably accurate to $\pm .02 \mathrm{~dB}$, resulting in a .04 $\mathrm{dB}$ error in the total apparent efficiency.

2. Connector and adapter errors. The connector on the input to the divider is a male TNC, and the connectors on the divider outputs are different miniature coaxial female 
types. Different adapters must therefore be used to connect the detector to the generator and output ports. The difference in loss in these adapters will contribute an additional error which is difficult to assess. It is probable that this error is less than .05 dB.

\section{Errors in the Mismatch Correction}

Both the amplitude and phase of each reflection coefficient must be known to completely correct for the mismatch correction; however, part of the correction may be determined from the amplitudes only. We can write the total mismatch correction $M$ as the product of two factors, $M=M_{1} M_{2}$, where

$$
M_{1}=\frac{1-\left|\Gamma_{\ell}\right|^{2}}{1-\left|\Gamma_{0}\right|^{2}}, \quad M_{2}=\frac{\left|1-\Gamma_{0} \Gamma_{g}\right|^{2}\left|1-\Gamma_{d} \Gamma_{i}\right|^{2}}{\left|1-\Gamma_{d} \Gamma_{g}\right|^{2}\left|1-\Gamma_{\ell} \Gamma_{i}\right|^{2}} .
$$

Only the magnitudes of $\Gamma_{\ell}$ and $\Gamma_{0}$ are required to determine $M_{1}$. The data supplied by the contractor gives,

$$
\begin{aligned}
& \left|\Gamma_{0}\right|=0.14 \text { to } 0.16 \\
& \left|\Gamma_{\ell}\right|=0.2 \text { to } 0.3
\end{aligned}
$$

This would make

$$
10 \log \mathrm{M}_{1}=-0.06 \mathrm{~dB} \text { to }-0.32 \mathrm{~dB}
$$

for the main power divider, and we will use $10 \log \mathrm{M}_{1}=$ $0.20 \mathrm{~dB} \pm 0.1 \mathrm{~dB}$.

This correction can and should be applied to the contractor's measurements to give a more accurate value for the efficiency. It could be computed more accurately by using the individual load and output reflection coefficients with the corresponding measured ratio to give

$$
\eta_{m}=M_{2} \quad \sum M_{1 i} R_{i},
$$

but there are other larger errors which make this small improvement pointless. 
The value of $M_{2}$ cannot be obtained without the phases of the I's, so we can only estimate its probable value. This uncertainty in $\mathrm{M}_{2}$ constitutes an uncertainty in the determination of $\eta_{m}$ and $\eta_{T}$. To determine the probable value for $M_{2}$ we have taken the measured magnitude of each $\Gamma$, taken all phase combinations in $20^{\circ}$ increments and computed the absolute value of the $\log$ of $\mathrm{M}_{2}$. This resulting distribution is shown in figure 3-2 for the following values of the I's.

$$
\begin{aligned}
& \left|\Gamma_{g}\right|=0.025 \\
& \left|\Gamma_{d}\right|=0.025 \\
& \left|\Gamma_{0}\right|=0.14-0.16 \\
& \left|\Gamma_{\ell}\right|=0.26 \\
& \left|\Gamma_{i}\right|=0.20
\end{aligned}
$$

The value of $\mathrm{M}_{2}$ is between zero and $\pm 0.53 \mathrm{~dB}$, and in some error analyses the maximum value is used. It is evident from figure 3-2 that it is improbable that $M_{2}$ would be as high as $\pm 0.53 \mathrm{~dB}$, but it is quite likely that the error is greater than $\pm 0.30 \mathrm{~dB}$. An estimate of $0.4 \mathrm{~dB}$ will be used for the following analysis.

Since $\Gamma_{\ell i}$ and $\Gamma_{i}$ are not known for the secondary dividers, accurate estimates of $M_{1}$ and $M_{2}$ cannot be made for these dividers. It seems probable that the $\Gamma_{i}$ 's will be about the same as for the main divider. The $\Gamma_{\ell i}{ }^{\prime} s$ will probably be larger due to the interaction between elements resulting in a larger uncertainty for both $\mathrm{M}_{1}$ and $\mathrm{M}_{2}$. Because of the above reasons, we will take

$$
\begin{aligned}
& 10 \log \mathrm{M}_{1}=-0.2 \mathrm{~dB} \pm 0.2 \mathrm{~dB}, \\
& \text { uncertainty in } 10 \log \mathrm{M}_{2}= \pm 0.5 \mathrm{~dB} .
\end{aligned}
$$

The individual measured or calculated quantities are summarized below to give the total efficiency and its associated uncertainty. 


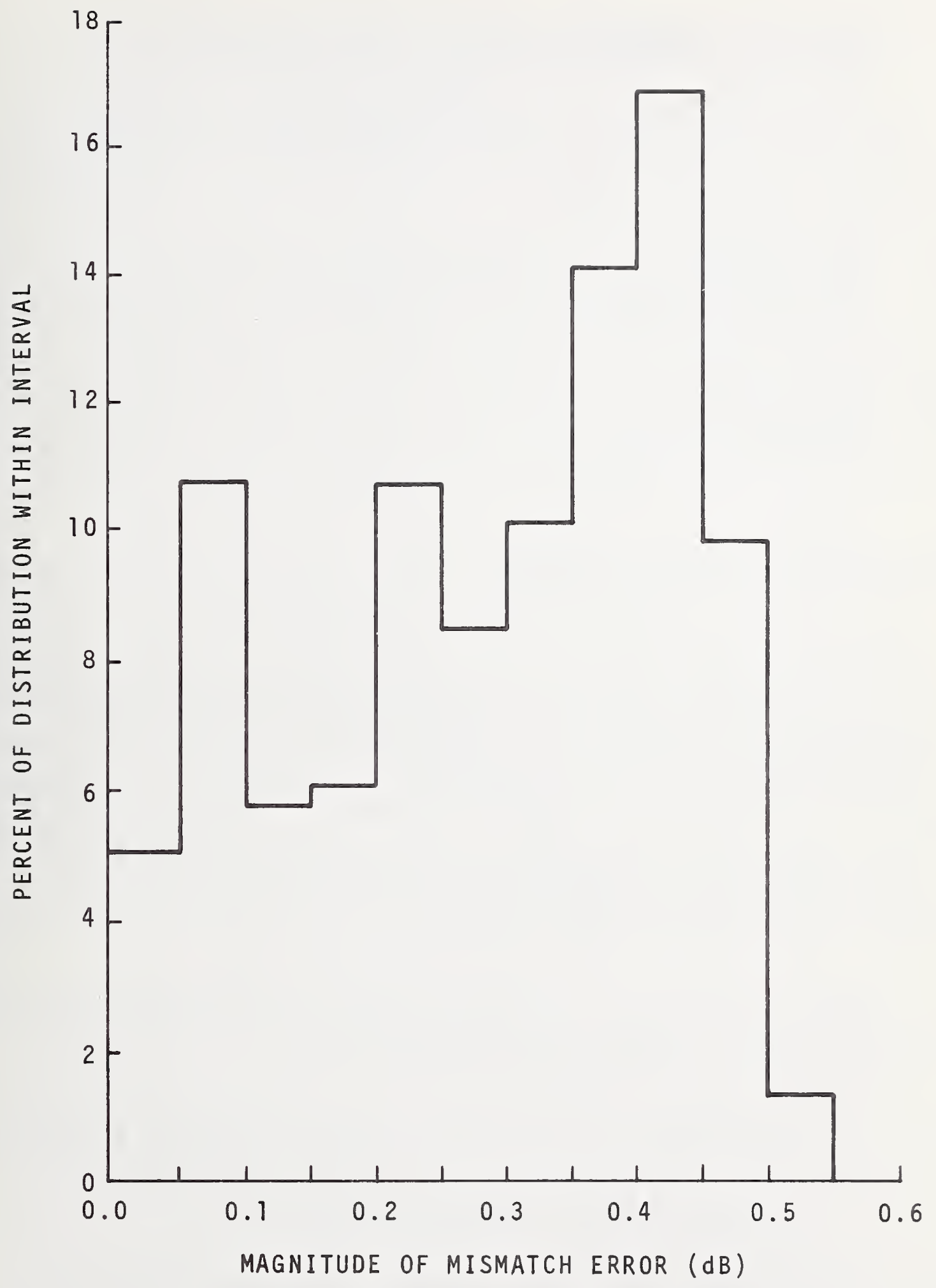

Figure 3-2. Distribution of Mismatch Errors for the S-194 Antenna System 
Table 3.1 Summary of Errors in Measured Efficiency

Measured or Calculated Value

(dB)

Apparent efficiency $=\eta_{m}^{\prime 2}$ Main divider

$\mathrm{M}_{1}$

Secondary divider

Main divider

$-0.284$

$-0.20$

$-0.20$

Uncertainty

$(\mathrm{d} B)$

$\pm 0.09$

$\pm 0.10$

$\pm 0.20$

$\pm 0.40$

$\mathrm{M}_{2}$

Secondary divider

TOTAL = $\pm 0.50$

$-0.68$

The total efficiency is then,

$$
n_{\mathrm{T}}=-0.68 \mathrm{~dB} \pm 0.68 \mathrm{~dB}
$$

A number of assumptions or approximations have been made during this discussion which were not discussed in detail. These assumptions were necessary for this approach on this antenna to arrive at a result, but they are not all good assumptions. The major approximations that have been made are summarized below to point out that the error may be even larger due to these approximations.

1. The losses in the radiative elements and radome have not been included, but they are probably small.

2. It is assumed that all nine dividers are identical. This is fairly well founded, since the apparent efficiencies of three different main dividers were quite close.

3. It is assumed that the coupling characteristics, the $\Gamma_{i}$ 's, the $b_{g i}$ 's, and $\Gamma_{0}$ are unchanged when the actual loads are 
replaced by the $50 \Omega$ terminations. This will not be true, and it would have been much better to make measurements with the actual loads in place. Since this was not done (and apparently was not possible), an additional error was introduced.

4. The $\Gamma_{i}$ 's and $\Gamma_{\ell i}$ 's are assumed to be independent of the output port. Data for each port of the main divider were available where $\left|\Gamma_{i}\right|$ was from 0.12 to 0.35 , and $\left|\Gamma_{\ell}\right|$ ranged from 0.20 to 0.30 , so there is a significant difference. The difference is probably even larger for the secondary dividers due to the cross coupling between radiating elements.

Even when these assumptions are used, and no error included to account for their approximate nature, this measurement approach still has an error of almost $\pm 0.7 \mathrm{~dB}$. We would therefore recommend that the results from this measurement not be used as an accurate value for antenna efficiency. Due to the nature of the antenna, measurements of gain and directivity or radiometric measurements are likely to yield a better result.

The results of the gain and directivity measurements, which will be discussed in the following sections, are

$$
\begin{aligned}
& \mathrm{G}=22.13 \mathrm{~dB} \pm 0.41 \mathrm{~dB}, \\
& \mathrm{D}=22.50 \mathrm{~dB} \pm 0.14 \mathrm{~dB} .
\end{aligned}
$$

These combine to give an antenna loss of $0.37 \mathrm{~dB}+0.50 \mathrm{~dB}$. If the gain error is reduced by further measurements as recommended in section 3.2 , the error in the loss would be reduced to about $\pm 0.25 \mathrm{~dB}$.

\subsection{Gain Measurements on the S-194}

Through discussion with the contractor the details of the gain measurement have been determined to be as follows. Measurements were made in a tapered anechoic chamber with a 
linearly polarized source antenna mounted at the tapered end of the chamber. The S-194 antenna was mounted on a model tower about 26 meters from the source, and a standard gain horn was also on the tower pointing in the opposite direction from the S-194. The output of the standard gain horn was connected to a waveguide-to-coax adapter which was connected to one input of a switch by a 0.5 meter piece of coaxial cable. The output of the S-194 was connected to the other input of the switch through a TNC to type $N$ adapter and a 0.5 meter piece of coaxial cable identical to the one used for the horn. The output of the switch was connected to a receiver through a coaxial cable about 30 meters long.

The S-194 was positioned for maximum received signal, the switch set to connect the S-194 output to the receiver, and the receiver gain set to obtain a relative $0.00 \mathrm{~dB}$ reading on the phase-gain indicator. The switch was then set to connect the standard gain horn to the receiver and the mount rotated to place the horn in position for maximum received signal. The attenuator in the receiver was decreased in 1 dB steps to bring the meter on scale and the difference from $z \in r o d B$ was read on the phase-gain indicator.

We will use the following notation to refer to the measurement parameters

$G=$ gain of the S-194 antenna

$\mathrm{d}=$ distance between source antenna and the S-194

$\Gamma_{a}=$ input reflection coefficient for the S-194

$\mathrm{S}_{11}, \mathrm{~S}_{12}, \mathrm{~S}_{22}=$ scattering parameters for transmission line between the S-194 and the receiver (composed of adapter, coax cable, switch and cable).

$\rho=$ polarization ratio for the S-194. This is a complex number equal to the ratio of the on-axis horizontal and vertical E-fields radiated from the S-194 antenna. 
Its magnitude would be equal to the axial ratio if the principal axis of the polarization ellipse were aligned along the horizontal or vertical directions.

$\alpha$ = available power ratio for the transmission line between the S-194 and receiver.

$\mathrm{P}_{\mathrm{d}}=$ power delivered to the receiver by the S-194.

Corresponding quantities for the standard gain horn will be denoted by primes, $G^{\prime}, d^{\prime}, \Gamma_{a}^{\prime}$, etc; and $\rho_{\mathrm{T}}=$ polari-

zation ratio for the transmitting antenna.

$\Gamma_{\ell}=$ Reflection coefficient at the receiver input port.

The ratio of net powers delivered to the receiver for farfield plane wave conditions is then given by,

$$
\frac{P_{d}}{P_{d}^{1}}=\frac{\alpha}{\alpha^{\prime}} \frac{\left(1-\left|S_{22}\right|^{2}\right)\left|1-S_{22}^{\prime} \Gamma_{\ell}\right|^{2}}{\left(1-\left|S_{22}^{\prime}\right|^{2}\right)\left|1-S_{22} \Gamma_{\ell}\right|^{2}} \frac{\left|1-\rho_{T^{2}}\right|^{2}\left(1+\left|\rho^{\prime}\right|^{2}\right)}{\left|1-\rho_{T^{\prime}} \rho^{\prime}\right|^{2}\left(1+|\rho|^{2}\right)} \frac{d^{\prime 2}}{d^{2}} \frac{G}{G^{1}} .
$$

The gain of the S-194 is then determined by measuring the power ratio with the receiver, measuring the two distances, knowing the gain of the standard, and making corrections for polarization and transmission line mismatch, non-identical $\alpha^{\prime} s$, and departure from far-field conditions.

The contractor supplied the following data,

$$
\begin{aligned}
10 \log \mathrm{G}^{\prime} & =16.45 \mathrm{~dB} \\
10 \log \frac{\mathrm{P}_{\mathrm{d}}}{\mathrm{P}_{\mathrm{d}}^{\prime}} & =4.90 \mathrm{~dB} \\
\mathrm{~d}^{\prime} & =28.44 \mathrm{~m} \quad \mathrm{~d}^{\prime}=26.0 \mathrm{~m} .
\end{aligned}
$$

No corrections were made by the contractor for the other terms in the gain equation, and the gain was determined to be,

$$
10 \log G=22.13 \mathrm{~dB}
$$

The various sources of error in the determination of $G$ are as follows:

1. Error in Measuring Power Ratio. 
The manufacturers specification for the receiver indicates that an error of $\pm 0.10 \mathrm{~dB}$ can be expected for a signal change of $5 \mathrm{~dB}$.

\section{Gain Standard Error.}

The NBS has found from experience in calibrating standard gain horns that the actual gain may differ by 0.2 to $0.3 \mathrm{~dB}$ from the values supplied by the manufacturer. Since the manufacturer's curve was used for the standard, an error of \pm 0.25 dB will be assumed.

3. Distance Error.

The distance was measured accurate to \pm 2 inches, which would cause a gain error of $\pm 0.02 \mathrm{~dB}$.

4. Transmission Line Mismatch.

With the long line between the switch and analyzer, the reflection coefficient looking back into the line ${\left(S_{22}\right.}_{2}$ and $S_{22}^{\prime}$ ) should be essentially the same for both antennas. This would mean essentially no error due to transmission line mismatch.

5. Polarization Mismatch.

The magnitude of this error will depend on the polarization ratio of all three antennas. No data on polarization ratios was taken for these antennas, so we will use some values for similar antennas and estimate a worst case error.

Table 3.2. Possible errors due to polarization mismatch

$\begin{array}{llll}\frac{\left|\rho_{\mathrm{T}}\right|}{.03} & \frac{\left|\rho^{\prime}\right|}{.03} & \frac{|\rho|}{.03} & \text { worst case error }(\mathrm{dB}) \\ .03 & .03 & .10 & \pm .02 \\ .10 & .03 & .10 & \pm .07 \\ .10 & .03 & .03 & \pm .15 \\ & & \pm .05\end{array}$

It is probable that the polarization mismatch error is about $0.05 \mathrm{~dB}$. 
6. Unequal $\alpha^{\prime} s$ for the Transmission Lines.

The $\alpha$ 's will be different due to the difference in adapters between the antennas and the coaxial line, and due to the different "generator" impedances seen by the two lines. The generator impedances in this case are the antenna reflection coefficients $\Gamma_{a}$ and $\Gamma_{a}^{\prime}$. The magnitude of $\Gamma_{a}$ is 0.20 , and $\Gamma_{a}^{\prime}$ for this type of horn antenna is about 0.03 . Substituting these values in equation (2-3), and assuming that the $S$-parameters are the same gives a difference of $0.2 \mathrm{~dB}$. An additional uncertainty of $0.05 \mathrm{~dB}$ will be added for the difference in adapters.

7. Multipath Errors.

The problem of scattering within the chamber, which is discussed in detail in section 3.3 .3 , suggests that there could be a sizable error in the gain measurements due to the scattering. The tapered chamber design produces a fairly uniform field in a given plane perpendicular to the chamber axes. But since that field is produced by the combination of the source antenna and its images, the field strength will vary as the source antenna is rotated about its axis, and this was observed in the pattern measurements. Moreover, the images increase the effective aperture of the source antenna. As a result, an inverse square law correction to account for different locations of the test and standard antennas may be of limited accuracy. It is very likely that there will also be a periodic variation in the field strength along the axis of the chamber due to the interaction of the actual source and its images. This effect will also produce an error in the gain measurements when the test and standard antennas are placed at different distances from the source as in these measurements. There is a lack of experimental data to confirm these suspected sources of error. The pattern data show variations when the antennas were rotated which indicate reflections no more than $20 \mathrm{~dB}$ below the direct 
signal. However, the chamber manufacturer claimed a "quiet zone" with reflections $40 \mathrm{~dB}$ below the direct signal. Where each of these factors is taken into account, an error of \pm 0.20 $\mathrm{dB}$ in the gain measurements is quite likely due to reflections within the chamber.

8. Near-Zone Error.

This error is due to insufficient separation distance between source and receiving antennas. In general it means that the gain measured at a given distance is lower than the actual far-field gain. The error is a function of the type and size of the antenna and the separation distance. For both test and standard antennas at the distances used, this error should be less than $0.05 \mathrm{~dB}$, and the net resulting error in $\mathrm{G}$ should be negligible.

9. Random Error.

Random error due to alignment, signal source stability, and receiver drift appears to have been $\pm 0.05 \mathrm{~dB}$. The resultant effect of these errors is summarized in Table 3.3 .

Table 3.3. Summary of errors in gain measurement.

Source of error

(1) Power Ratio Measurement

(2) Standard Gain Horn gain

(3) Distance Measurement

(4) Transmission Line Mismatch

(5) Polarization Mismatch

(6) Unequal $\alpha^{\prime} s$

(7) Multipath Signals

(8) Near-Zone

(9) Random Error

Resultant Quadrature Sum

\section{Error in gain (dB)}

$\pm 0.10$

$\pm 0.25$

$\pm 0.02$

0.00

$\pm 0.05$

$\pm 0.25$

$\pm 0.20$

0.00

$\pm 0.05$

$\pm 0.41 \mathrm{~dB}$

*The expression "quadrature sum" is the square root of the sum of the squares of the individual errors. 
A few of these errors could be reduced without further measurement on the S-194 antenna. The standard gain horn could be calibrated and its axial ratio measured. This horn could then be used to measure the axial ratio of the source antenna, which would reduce the uncertainty due to polarization mismatch. The reflection coefficient of the horn could be measured, and also the characteristics of the adapters if they are available. This information would reduce error number 6 .

If these steps were taken, it is probable that the gain error would be reduced to about $\pm 0.20 \mathrm{~dB}$. If this $\pm 0.2 \mathrm{~dB}$ gain error is unacceptable, then new gain measurements could be made at the NBS to reduce the error to about $\pm 0.10 \mathrm{~dB}$.

\subsection{Antenna Pattern, Beam Efficiency, and Directivity on the S-194 Antenna}

\subsubsection{Introduction}

The S-194 antenna was measured on a "model mount" using a source antenna which is a dipole backed by a symmetrical ground plane. In the model mount, shown in figure $3-3$, the antenna is mounted on a nominally horizontal "head" axis ( $z$ axis) above an orthogonal (nominally vertical) "A" axis. The antenna was mounted with its figure axis nominally coincident with the head axis, the antenna rotated by an angle (called $\phi$ ) about the head axis and then about the vertical $A$ axis by an angle called $\theta$. Care was used in aligning the antennas. Thus, to eliminate antenna load deflection, a mirror and a weight equal to that of the antenna (35 lbs) were mounted on the head and adjustments made with the aid of a theodolite. Since the rated operating load of the mount is at least $75 \mathrm{lbs}$ and the 


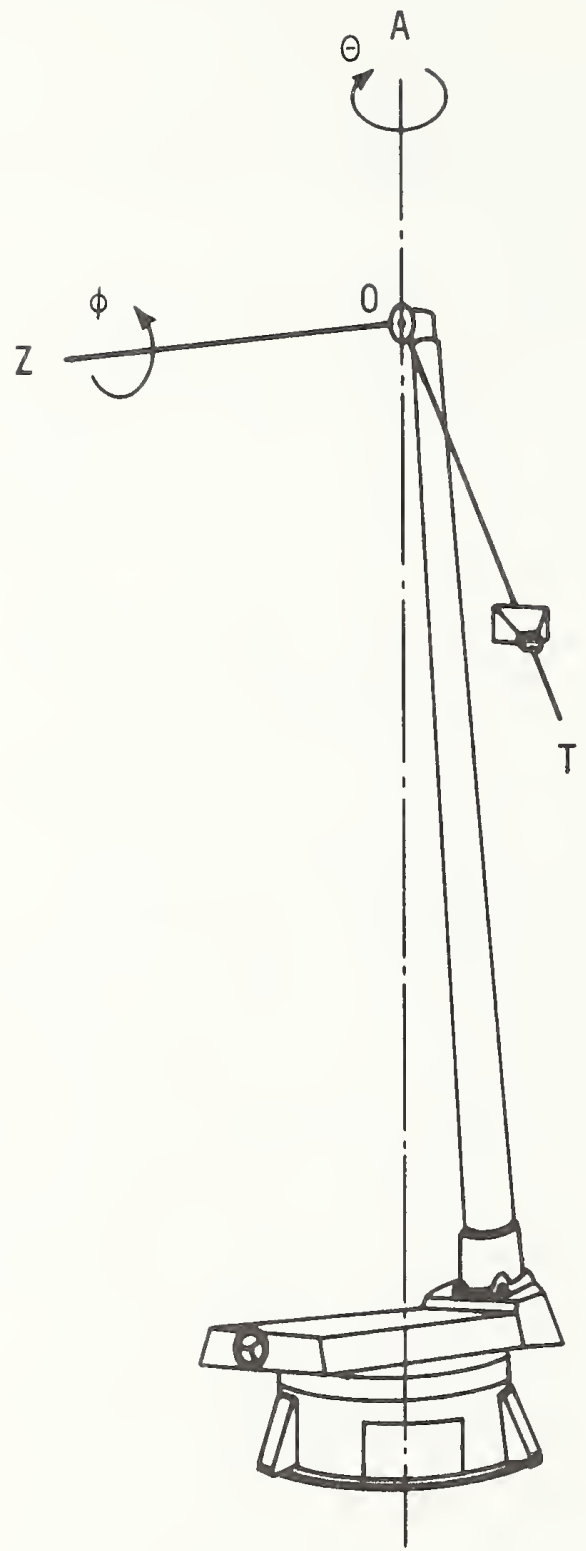

Figure 3-3. Model Mount Used for S-194 Antenna Measurements 
head offset may be adjusted, deflection should have been negligible. (According to the manufacturer's specification, deflection is less than $2^{\circ}$ at full load without compensation.)

With the test antenna on boresight $(\theta=0)$, the source antenna was rotated about the inter-antenna axis to give minimum response and then rotated by $\pi / 2$ for maximum response. For a given cut ( $\phi$ value), the orientations of the source antenna giving maximum and minimum response on boresight $(\theta=0)$ were used to measure the "principal" and "cross" polarization, respectively, for the entire cut. The minimum may of course be located more accurately than the maximum because the fractional numerical change in the slope is greater. Moreover, as will be explained, the maxima and minima are separated by exactly $\pi / 2$ for arbitrary polarization, neglecting scattering from the chamber walls and imprecise alignment.

Nevertheless, anomalies did occur in the data. The reported cross polarization was commonly maximum (only $20 \mathrm{~dB}$ below the principal polarization), at or near boresight, but, as will be explained, it should be nominally zero there. Further, cross polarization reported on boresight varied with the cut ( $\phi$ fixed, $\theta$ variable), varying from 18.7 to more than $35 \mathrm{~dB}$ down from the principal polarization for the same antenna. To investigate these inconsistencies, a simple experiment was performed. With the test antenna fixed on boresight $(\theta=0)$, the minimum response (cross polarization) varied from 18 to more than $35 \mathrm{~dB}$ below the maximum response (main polarization), depending upon rotation of the antenna pair (about the inter-antenna axis) with respect to the anechoic chamber. Similarly, the maximum response varied by $2 \mathrm{~dB}$ depending upon the rotation of the antenna pair with respect to the chamber. Consequently, critical evaluation of the data is required, using auxiliary information to resolve these inconsistencies. 


\subsubsection{Properties of the Model Mount}

The received signal as a function of the model mount angles must first be related to the antenna pattern and polarization properties. Assuming far field conditions, the complex received signal is proportional to $\underline{T} \cdot \underline{R}$ where $\underline{T}$ represents the transmitting properties of the source antenna and $\underline{R}$ is a similar vector representing the receiving properties of the test antenna. Both $\underline{T}$ and $\underline{R}$ are functions of the direction of propagation and everywhere perpendicular to it. T is proportional to the far field electric vector of the source antenna and, if the receiving antenna is reciprocal, $\underline{\mathrm{R}}$ is proportional to the electric vector arising from the test antenna when it is acting as a transmitter. Hence, we require $\underline{R}$, its magnitude, or the magnitudes of its components as functions of the orientation of the test antenna, given I on the axis of the source antenna for two different source orientations and the received signal as a function of the model mount angles.

First consider the right-handed rectangular coordinate system Oxyz and the spherical polar system $\theta, \Phi, r$, both fixed in the test antenna and related as shown in figure 3-4, with unit vectors $\underline{e}_{\ominus}$, $\underline{e}_{\Phi}$, and $\underline{e}_{r}$. (These angles should not be confused with the model mount angles $\theta$ and $\phi_{\cdot}$ ) As may be seen from the figure, the components are related by

$$
\underline{s}=A \underline{x},
$$

where $\underline{s}$ and $\underline{x}$ are the column matrices $\left\{R_{\Theta}, R_{\Phi}, R_{r}\right\}$ and $\left\{R_{x}, R_{y}, R_{z}\right\}$,

$$
A=\left(\begin{array}{llr}
c C & s C & -S \\
-s & c & 0 \\
c S & s S & C
\end{array}\right),
$$

and S, C, S, and C indicate $\sin \Phi, \cos \Phi, \sin \theta$, and $\cos \theta$, respectively. Since $A$ is an orthogonal matrix, 


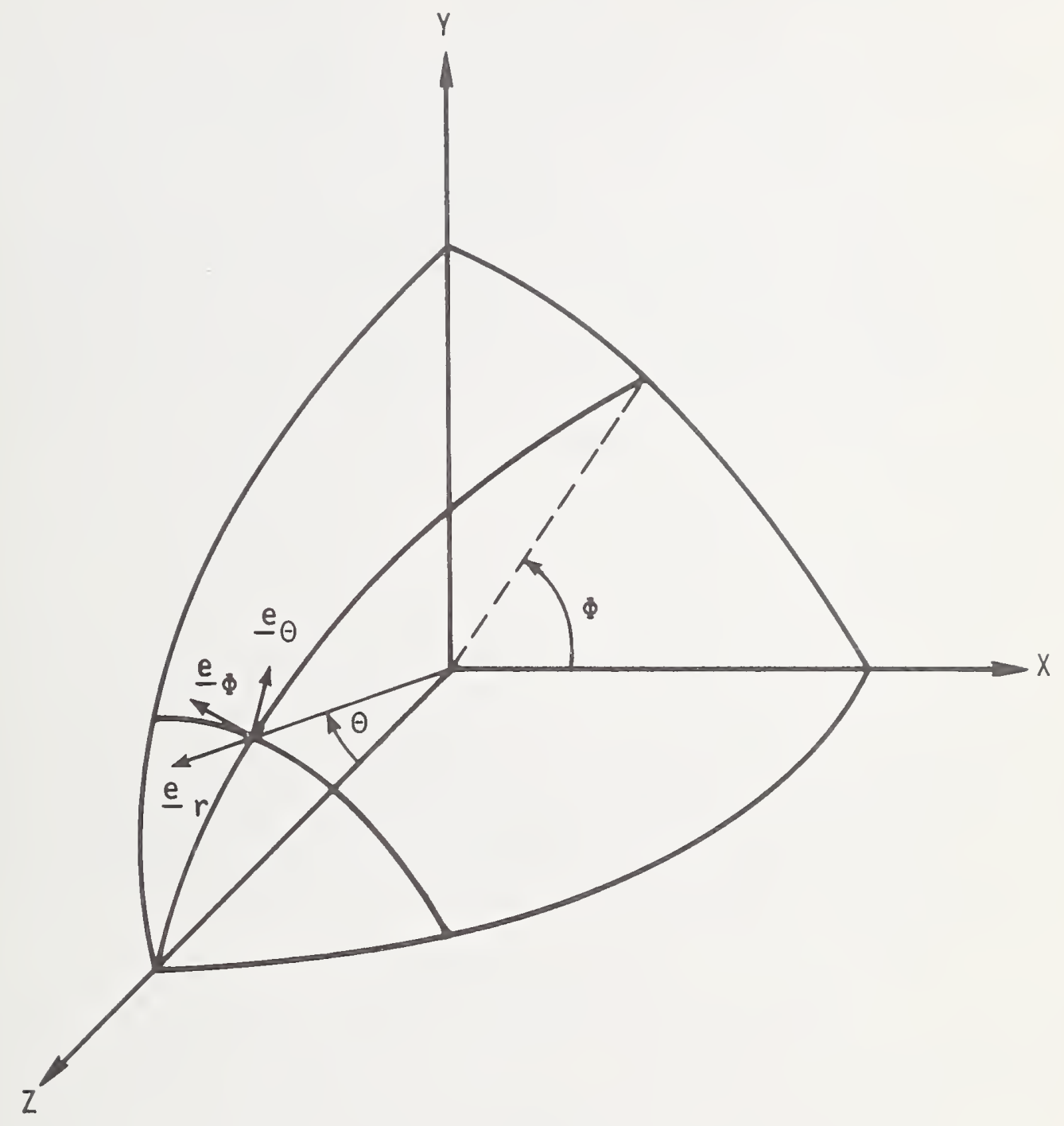

Figure 3-4. Antenna-fixed Coordinate Systems 


$$
\underline{x}=\tilde{A} \underline{s},
$$

where $\sim$ indicates the transpose. The components $R_{x}, R_{y}$, and $\mathrm{R}_{z}$ relative to the antenna-fixed rectangular coordinate system are required in terms of the experimental data.

It is assumed that: (a) the head and $A$ axes intersect and are perpendicular to each other, and (b) the axis of rotation of the source antenna passes through their intersection and coincides with the head axis for $\theta=0$. For the initial model mount configuration $(\theta=\phi=0), y$ and $z$ of $0 x y z$ (the antennafixed system) are coincident with the $A$ and head axes, respectively, and $0 x y z$ is coincident with the space-fixed coordinate system OXYZ. Then, as may be seen from figures 3-3 and 3-4, rotation of the head axis by $\phi=-\Phi$ followed by rotation about the vertical axis by $\theta=\theta$ yields the relations

$\mathrm{R}_{\Theta}(\theta, \Phi)=\mathrm{R}_{X}(\theta, \phi), \mathrm{R}_{\Phi}(\theta, \Phi)=\mathrm{R}_{Y}(\theta, \phi)$, and $\mathrm{R}_{\mathrm{Y}}(\theta, \Phi)=\mathrm{R}_{Z}(\theta, \phi)$,

where $R_{X}, R_{Y}$, and $R_{Z}$ are the components of $\underline{R}$ in the indicated directions in the space-fixed coordinate system after the above rotation by $\theta$ and $\phi$. Since the source antenna is also rotated by $\phi=-\Phi$,

$$
\underline{s}=\underline{X}=B \underline{x}^{\prime},
$$

where

$$
B=\left(\begin{array}{rrr}
c & s & 0 \\
-s & c & 0 \\
0 & 0 & 1
\end{array}\right)
$$

and $\underline{x}$ and $\underline{x}^{\prime}$ are the components of $R$ in the coordinates fixed in space (3-21) and the source antenna, respectively. Hence,

$$
\underline{x}^{\prime}=\tilde{B} \underline{x}=\tilde{B} \underline{s}=\tilde{B} A \underline{x} \equiv M \underline{x},
$$


where

$$
M=\left(\begin{array}{ccc}
c^{2} C+s^{2} & s c(C-1) & -c S \\
s c(C-1) & s^{2} C+c^{2} & -s S \\
c S & s S & C
\end{array}\right) .
$$

Since $A$ and $B$ are orthogonal, so is $M$ and

$$
\underline{x}=\tilde{M} \underline{x}^{\prime} \text {. }
$$

Since $R_{r}=0$ in the far field, the bottom row of (3-19) gives $R_{z}$ in terms of $R_{x}$ and $R_{y}$. Substituting this value of $R_{z}$ in (3-24), one obtains an alternative expression for $M$, namely

$$
M^{\prime}=\left(\begin{array}{ccc}
c^{2} \Sigma+s^{2} & s c(\Sigma-1) & 0 \\
s c(\Sigma-1) & s^{2} \Sigma+c^{2} & 0 \\
0 & 0 & 0
\end{array}\right),
$$

where $\Sigma$ represents $\sec \theta$. Since $M^{\prime}$ has no inverse it may not be used in (3-26) but a similar simplification results from the fact that the last column of $M$ may be ignored since $R_{z^{\prime}}=0$. Thus, (3-24) and following relate the components of $R$ for the coordinate system fixed in the test antenna to those for the system fixed in the source antenna. In particular, $\mathrm{R}_{\mathrm{z}^{\prime}}=0$ and, for a calibrated 1 inearly polarized source antenna, $R_{x}$, and $R_{y}$, i.e., the dot product of a row of $(3-25)$ or $(3-27)$ with $x$, may be considered as known.

The qualitative character of $M$ is shown by its behavior on meridian cuts (fixed $\Phi$ ) corresponding to the $E$ plane $(\Phi=0$ or $\pi)$, the $H$ plane $(\Phi=\pi / 2$ or $3 \pi / 2)$, and the two diagonal planes $(\Phi=\pi / 4,3 \pi / 4,5 \pi / 4$, or $7 \pi / 4)$. The corresponding $M^{\prime} s$ are respectively:

$$
\left(\begin{array}{rrr}
C & 0 & \mp S \\
0 & 1 & 0 \\
\pm S & 0 & C
\end{array}\right)
$$




$$
\begin{aligned}
& \left(\begin{array}{rrr}
1 & 0 & 0 \\
0 & C & \mp S \\
0 & \pm S & C
\end{array}\right), \\
& \left(\begin{array}{lll}
\frac{1}{2}(C+1) & \frac{e}{\sqrt{2}}(C-1) & \frac{-f}{\sqrt{2}} S \\
\frac{e}{\sqrt{2}}(C-1) & \frac{1}{2}(C+1) & \frac{-g}{\sqrt{2}} S \\
\frac{f}{\sqrt{2}} S & \frac{g}{\sqrt{2}} S & C
\end{array}\right) .
\end{aligned}
$$

If $\theta=0$, all three matrices reduce to the unit matrix.

In (3-28) e, $f$, and $g$ are each \pm 1 depending upon the individual angle; $f g=e ;$ and, for the first two cases, the upper signs hold for $\Phi=0$ or $\pi / 2$ and the lower signs for $\Phi=\pi$ or $3 \pi / 2$. The alternate M's (corresponding to (3-27)), are obtained by replacing the last row and column by zeros and the remaining $C^{\prime} \mathrm{s}$ by $\Sigma$ 's. On the $\mathrm{E}$ and $\mathrm{H}$ planes, only one element of each row of $\tilde{M}$ is non-zero and that element is equal to $C, 1$, or $\pm S$; hence, $R_{x}, R_{y}$, and $R_{z}$ may be determined by a single meas urement with a linearly polarized antenna. Further, on these planes, their absolute values may be determined without a phase measurement. For the diagonal and most other planes the components are mixed so that phase measurements must be made to obtain the individual components for $\theta \neq 0$, although the magnitudes of $R_{x}$ and $R_{y}$ may be obtained to fair accuracy without phase measurements if $\theta$ is small. The magnitude of $\underline{R}$ (without reference to polarization) may of course be obtained without phase measurements.

Commonly the principal and cross polarizations are not referred to a direction fixed with respect to the antenna but rather, for each orientation of the test antenna in its mount, chosen perpendicular to the line of antenna centers, 
say vertical and horizontal. Such a description depends upon the mount and the manner in which the antenna is mounted on the mount, but is quite practical if it is given in terms of the mount and mounting used with the antenna in its eventual application. However, it is commonly given in terms of the mount and antenna mounting used for measurement. (The discrepancies may be small for directions not far from boresight.) In the absence of information concerning the mount to be used, the measurement mount is treated. For the model mount, manner of antenna mounting, and coordinate systems used here, the principal and cross polarizations could have been taken to be $R_{\Theta}$ and $R_{\Phi}$ respectively. These polarizations would have been obtained directly with a linearly polarized antenna if the source had not been rotated. However, since the source antenna was rotated, $\underline{s}=B \underline{x}^{\prime}(3-22)$ and $\underline{x}^{\prime}=B \underline{s}$. For the cases described by equation $(3-28)$, B reduces to

$$
\left(\begin{array}{rrr} 
\pm 1 & 0 & 0 \\
0 & \pm 1 & 0 \\
0 & 0 & 1
\end{array}\right),\left(\begin{array}{lrl}
0 & \pm 1 & 0 \\
\mp 1 & 0 & 0 \\
0 & 0 & 1
\end{array}\right) \text {, and }\left(\begin{array}{ccc}
\alpha / \sqrt{2} & \beta / \sqrt{2} & 0 \\
-\beta / \sqrt{2} & \alpha / \sqrt{2} & 0 \\
0 & 0 & 1
\end{array}\right)
$$

respectively, where $\alpha$ and $\beta$ are \pm 1 depending upon the angle.

\subsubsection{Symmetry, Polarization, and Scattering}

Determination of the antenna polarizations on boresight and evaluation of chamber scattering require a careful interpretation of the data and its anomalies together with a priori considerations. The source antenna and the S-194 antenna each have two orthogonal planes of nominal material symmetry and are nominally excited (in the transmitting mode) so that one plane (the E plane) is a plane of field symmetry and the other (the $\mathrm{H}$ plane) is a plane of field antisymmetry. Field symmetry is used here in the vector-pseudovector sense, 
i.e., reflection of an electric field vector $\underline{E}$ (solid arrow in Fig. 3-5) in a plane perpendicular to the vector reverses its direction, while reflection of a magnetic field pseudovector H (dashed arrow in Fig. 3-5) in a plane perpendicular to the usual arrow leaves the field unchanged, i.e., the pseudovector $\underline{H}$ behaves like the elementary current loop associated with $\underline{H}$.

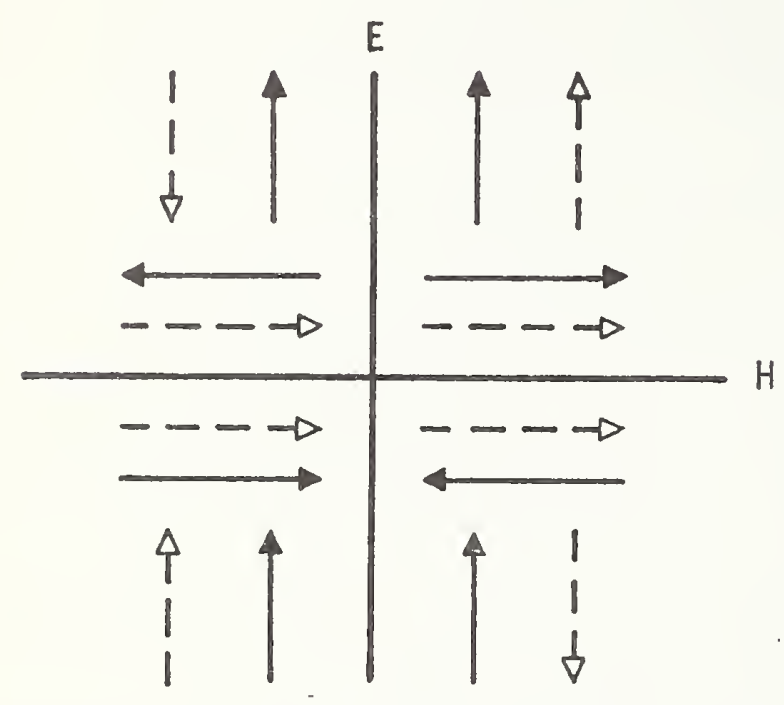

Figure 3-5. Antenna Field Symmetries

In this sense, the E field and the associated $\underline{H}$ (more precisely magnetic induction B) field (as functions of position) have identical symmetry properties. For an antenna with such symmetry, on the $\mathrm{E}$ plane there can be no $\underline{E}$ component normal to the plane and on the $\mathrm{H}$ plane there can be no $\mathrm{E}$ component in the plane. Thus, any antenna with such symmetry must be linearly polarized with no cross polarization on the two planes and doubly so on their intersection (boresight). These statements apply to (a) the radiated field (a function of position), (b) the complex vector radiation pattern $\underline{T}$ (a function of direction), (c) the complex vector receiving pattern $\underline{R}$, and (d) the corresponding power patterns. Moreover, if the anechoic chamber in which the antennas were measured had two planes of material symmetry and the source antenna was symmetrically positioned and oriented, then the total radiation incident upon the test (S-194) antenna (including the radiation scattered from the chamber walls) had the aforementioned polarization character (linear, no cross polarization). Moreover, $R_{\Phi}$ must be zero in the $E$ plane and $R_{\Theta}$ must be zero in the $H$ plane. 
The reported cross polarization on the $\mathrm{H}$ plane cut is consistent with the nominal symmetry, being at least $35 \mathrm{~dB}$ down on boresight and at least $30 \mathrm{~dB}$ down for every value of $\theta$. However, the cross polarization reported for the E plane and diagonal cuts are maximum on or near boresight $(\theta=0)$, being only 18.7 to approximately $20 \mathrm{~dB}$ down from the principal polarization on boresight. The latter meas urements are not only inconsistent with the nominal symmetry but at wide variance with the other values on boresight. Further, these boresight differences must be due to the difference in the field scattered by the anechoic chamber.

To estimate the ellipticity of the polarization of the test antenna and the radiation incident upon it, the boresight measurements are used, particularly the fact that for one pair of orientations the minimum response was more than $35 \mathrm{~dB}$ below the maximum response. (Recall that no ambiguity arises from the model mount behavior for $\theta=0$. See $(3-28)$. For two antennas, each in the far field of the other and one rotating about the line connecting the centers of the antennas, the received power (proportional to $|\underline{T} \cdot \underline{R}|^{2}$ ) may be shown to be proportional to $(1-t r)^{2} \cos ^{2} \alpha+(t-r)^{2} \sin ^{2} \alpha$, where $\alpha$ is the angle between the major axes of the two polarization ellipses, $r$ is the ratio of minor axis to the major axis for the receiving antenna, and $t$ is the corresponding ratio for the trans mitting antenna. (The ratios are allowed to assume negative as well as positive values to account for the senses of rotation.) For antennas which are even approximately linearly polarized, $t$ and $r$ are small enough compared to unity that the minimum response will be proportional to $(t-r)^{2}$, not $(1-t r)^{2}$. Due to the difference in the character of the two antennas, $r / t$ will not be even roughly +1 except by chance. Thus, the small magnitude of $(t-r)^{2}$ is probably not due to cancellation so that $t^{2}$ and $r^{2}$ are probably no more than a few dB greater than $(t-r)^{2}$. 
The scattered field (and therefore the total radiation incident upon the test antenna) depends only upon the orientation of the source antenna and so upon $\phi$ but not $\theta$; however the effect of the scattered field may depend upon $\theta$, say as the main beam points toward a scatterer. The data for the $\mathrm{H}$ plane cut indicates that $\mathrm{r}^{2}$ for the test antenna on the boresight and $t^{2}$ for the composite radiation field when the source antenna is horizontally oriented are probably no greater than $-30 \mathrm{~dB}$. Hence, the large values of $(t-r)^{2}$ for the source antenna oriented vertically or diagonally must be due essentially to the effective $t^{2}$ which is therefore approximately -18 or $-20 \mathrm{~dB}$ for these source orientations.

Further, the reported cross polarization on or near boresight must be due primarily to scattered radiation except for the $\mathrm{H}$ plane measurements. The fact that the maxima for other source orientations occur on or near boresight is in agreement with this conclusion since the reflection coefficient tends to increase as the incident radiation approaches a grazing angle. (For an infinite plane wave incident upon an infinite plane surface, both the perpendicular and parallel reflection coefficients approach unity as the grazing angle is approached, the perpendicular coefficient being fairly large for angles well off grazing.) Moreover, the fact that little if any "cross polarization" occurs for $|\theta|>14^{\circ}$ indicates that the direction of the scattered radiation does not deviate far from the line of antenna centers. It should be noted that the reported values of cross polarization do not indicate the magnitude of the total scattered power but only a rough lower bound; the scattered power is probably significantly larger than the bound, particularly when the source antenna is polarized roughly horizontally or vertically. The circumstance with the anechoic chamber may be much more common than is generally realized. The reflection coefficient was reported 
to be down by at least $40 \mathrm{~dB}$, and therefore scattering was considered to be negligible. Such values are of course for normal incidence and may be of limited significance for a tapered anechoic chamber.

\subsubsection{Error Estimates}

Since the preceding analysis indicates that the measurements of cross polarization have little if any value, the following discussion is limited to the total received power as a function of direction. The following sources of error are considered:

1. Non-linear polarization of the test antenna and composite source on boresight. The previously estimated magnitudes of $r$ and $t(0.03$ and 0.10$)$, give rise to an error of $\pm 0.03 \mathrm{~dB}$ in the directivity and $\pm 0.14 \%$ in the beam efficiency.

2. Receiver non-linearity. Assuming a non-linearity of $0.02 \mathrm{~dB} / \mathrm{dB}$ yields an error of $0.08 \mathrm{~dB}$ in the directivity and an error of $0.24 \%$ in the beam efficiency.

3. Scattered signal. To estimate the error due to scattered radiation, the reported cross polarization for the source antenna vertically polarized was used as a measure of the scattered radiation. The resulting errors in the directivity and beam efficiency were found to be $0.87 \mathrm{~dB}$ and $0.6 \%$, respectively. To the extent that the total radiation incident upon the test antenna propagates parallel to the line of antenna centers, renormalization of the values for each cut to yield the same value on boresight yields correct relative pattern values. Using the same estimate for the scattered radiation, which estimate assumes off-axis scattered radiation, errors of $0.03 \mathrm{~dB}$ and $0.5 \%$ were estimated for renormalized measurements as carried out by the contractor. The total scattered signal could not be determined from the available data, so the following two assumptions were 
made. (1) That part of the scattered signal for which there was a change of polarization was used as a lower bound, yielding a rough lower bound for the error due to scattering. (2) As a measure of the scattered signal with the change of polarization, the maximum value was used, namely that associated with vertical polarization of the source antenna. (For other orientations of the source antenna, actual values of the scattered signals with change of polarization were less.) It is hoped that these two assumptions, the first leading to a low error estimate and the second toward a high estimate, will roughly cancel each other.

Table 3.4. Summary of Errors in Directivity and Beam Efficiency

$\begin{array}{lll}\text { Source of Error } & \frac{\Delta \mathrm{D}}{\Delta \varepsilon} \\ \text { Polarization } & \pm 0.03 & \pm 0.14 \\ \text { Non-linear Receiver } & \pm 0.08 & \pm 0.24 \\ \text { Scattered Power } & \pm 0.03 & \pm 0.50 \\ \quad \text { Total } & \pm 0.14 \mathrm{~dB} & \pm 0.88 \%\end{array}$

\subsection{Calibration of the S-194 Radiometer}

Briefly, it is the contractor's estimate that the S-194 radiometer can measure brightness temperature with a probable error of \pm 1.7 to $\pm 6.3 \mathrm{~K}$. We feel that the errors are in the range from \pm 14 to $\pm 42 \mathrm{~K}$. The difference in estimates is due primarily to the uncertainty assigned to the accuracy in meas uring antenna efficiency and in the accuracy of the standards used to calibrate the radiometer.

In the S-194 radiometer analysis, some concern was expressed by Hartsfield about the mathematics of joining an antenna to a receiver, or even if a satisfactory expression existed. To answer the quesions raised, a more rigorous joining equation than the one presented by the contractor is included and utilized in the error analysis. 


\subsubsection{Description of the S-194 Radiometer}

A diagram of the S-194 system is shown in Fig. 3-6. Radiation is incident on the antenna with brightness temperature $\mathrm{T}_{\mathrm{B}}$. A digital output, $A_{A}$, is obtained that is linearly related to the incident radiation; viz.

$$
T_{B}=K^{\prime \prime \prime} A_{A}-T_{0}^{\prime \prime \prime} \text {, }
$$

where the slope $\mathrm{K}^{\prime \prime \prime}$ and the intercept $\mathrm{T}_{0}^{\prime \prime}$ are determined in part by removing the antenna and utilizing standard noise generators. To protect the calibration against internal gain changes, a comparison radiometer of the switching type was selected; and to protect against time varying internal noise contributions, hot and cold internal reference standards and various temperature-monitoring sensors are available for an update calibration routine. The accuracy to which $\mathrm{T}_{B}$ is known for a given $A_{A}$ depends on (1) the radiometer's resolution, (2) the initial accuracy of the calibration for the radiometer, (3) the stability and/or the accuracy of the update corrections, and (4) the accuracy of characterizing the antenna.

When the antenna is replaced with standard noise generators,

$$
\mathrm{T}_{\mathrm{STD}}=\mathrm{KA}_{\mathrm{STD}}-\mathrm{T}_{0} \text {. }
$$

The values for $K$ and $T_{0}$ depend on the reflection coefficients of the standards $\Gamma_{S T D}$, in addition to the circuit between the reference planes A and X (Fig. 3-6). To evaluate this dependence, note that the output d.c. voltage, E, at X (see fig. 3-6) is related to the power available at the antenna input at $A$ by

$$
E=Q_{X A} T_{S T D}+Q_{X F}\left(\Theta_{F A}+T_{A M P}\right)
$$

where

$$
Q_{X i} \equiv \frac{\text { Voltage at } X}{\text { Power available at i }}
$$


and $i$ refers to the reference planes $A, F$ and $R . T_{A M P}$ is the effective input noise temperature of the amplifier system, and $\theta_{F A}$ is the radiation (in degrees kelvin) originating in the front end of the radiometer and available to the amplifier at F. When the radiometer is switched to the reference generator, the dc voltage at $X$ is adjusted via the binary attenuator to equal $E$ in $(3-32)$,

$$
E=Q_{X R}^{\prime \prime} T_{R}+Q_{X F}^{\prime \prime}\left(\theta_{F R}+T_{A M P}^{\prime \prime}\right) \text {, }
$$

where the symbols have meanings similar to those in (3-32). The double prime implies the switch is connecting the reference source. Using the available power ratio coefficients defined in $(2-2)$,

$$
\begin{aligned}
& Q_{X R}^{\prime \prime}=Q_{X F}^{\prime \prime} \alpha_{F R} \equiv \\
& \left\{\frac{\text { Voltage at X }}{\text { Power available at } F}\right)\left(\frac{\text { Power available at } F}{\text { Power available at } R}\right),
\end{aligned}
$$

and $(3-34)$ becomes

$$
E=Q_{X F}^{\prime \prime}\left[\alpha_{F R} T_{R}+\theta_{F R}+T_{A M P}^{\prime \prime}\right]
$$

Equating (3-36) with (3-32), comparing with $(3-31)$, and noting that $\mathrm{Q}_{\mathrm{XA}} / \mathrm{Q}_{\mathrm{XF}}=\alpha_{\mathrm{FA}}$ via a relationship similar to $(3-35)$,

and

$$
K=\frac{\left[\alpha_{F R} T_{R}+\theta_{F R}+T_{A M P}^{\prime \prime}\right]}{a \alpha_{F A}}
$$

$$
\mathrm{T}_{0}=\frac{\mathrm{a}\left[\Theta_{\mathrm{FA}}+\mathrm{T}_{\mathrm{AMP}}\right]+\mathrm{b}\left[\alpha_{F R} \mathrm{~T}_{\mathrm{R}}+\Theta_{\mathrm{FR}}+\mathrm{T}_{\mathrm{AMP}}^{\prime \prime}\right]}{\mathrm{a} \alpha_{\mathrm{FA}}}
$$

where $a$ and $b$ are defined by the linear relation between $A_{S T D}$ and the binary attenuation controlling the voltages at $X$; namely 


$$
A_{S T D}=a\left(Q_{X F}^{\prime \prime} / Q_{X F}\right)+b
$$

If the isolation between ports on the diode switch is adequate, then the terms within the square brackets are independent of the impedance at the input to the radiometer, and the change of $K$ and $T_{0}$ to $K^{\prime}$ and $T_{0}^{\prime}$, due to a changed input impedance is governed by

$$
K^{\prime}=\mathrm{R} K \text {. }
$$

Hence

$$
\mathrm{T}_{0}^{\prime}=\mathrm{R}_{0}+\frac{\left(\Theta_{\mathrm{FA}}^{\prime}{ }^{-}{ }_{\mathrm{FA}}\right)+\left(\mathrm{T}_{\mathrm{AMP}}^{\prime}{ }^{\left.-\mathrm{T}_{\mathrm{AMP}}\right)}\right.}{\alpha_{\mathrm{FA}}^{\prime}}
$$

where $\mathrm{R} \equiv \alpha_{\mathrm{FA}} / \alpha_{\mathrm{FA}}$, and the prime indicates the parameters associated with a new impedance. If we use a more convenient form of $(2-3)$, namely

$$
\alpha_{i j}=\frac{\left(1-\left|\Gamma_{j}\right|^{2}\right)\left|s_{i j}\right|^{2}}{\left(1-\left|\hat{\Gamma}_{i}\right|^{2}\right)\left|1-S_{i j} \Gamma_{j}\right|^{2}},
$$

where $\Gamma_{j}$ is the reflection coefficient looking away from the two-port at port $j$, and $\hat{\Gamma}_{j}$ is the reflection coefficient looking into the two-port at port $j$, then

$$
\mathrm{R}=\frac{\left(1-\left|\Gamma_{\mathrm{STD}}\right|^{2}\right)\left|1-\Gamma_{\mathrm{ANT}} \Gamma_{\mathrm{SW}}\right|^{2}\left(1-\left|\Gamma_{\mathrm{F}}^{1}\right|^{2}\right)}{\left(1-\left|\Gamma_{\mathrm{ANT}}\right|^{2}\right)\left|1-\Gamma_{\mathrm{STD}} \Gamma_{\mathrm{SW}}\right|^{2}\left(1-\left|\Gamma_{\mathrm{F}}\right|^{2}\right)} \text {. }
$$

The unprimed parameters correspond to the impedance of the standards used to calibrate the radiometer and the primed parameters to the impedance of the antenna. $\Gamma_{F}$ is the reflection coefficient looking into the filter at reference plane F; $\Gamma_{\text {STD }}$ is the reflection coefficient of the standard; $\Gamma_{\text {ANT }}$ is the reflection coefficient of the antenna; and $\Gamma_{S W}$ is the reflection coefficient looking towards the switch at reference plane A (see Fig. 3-6). 


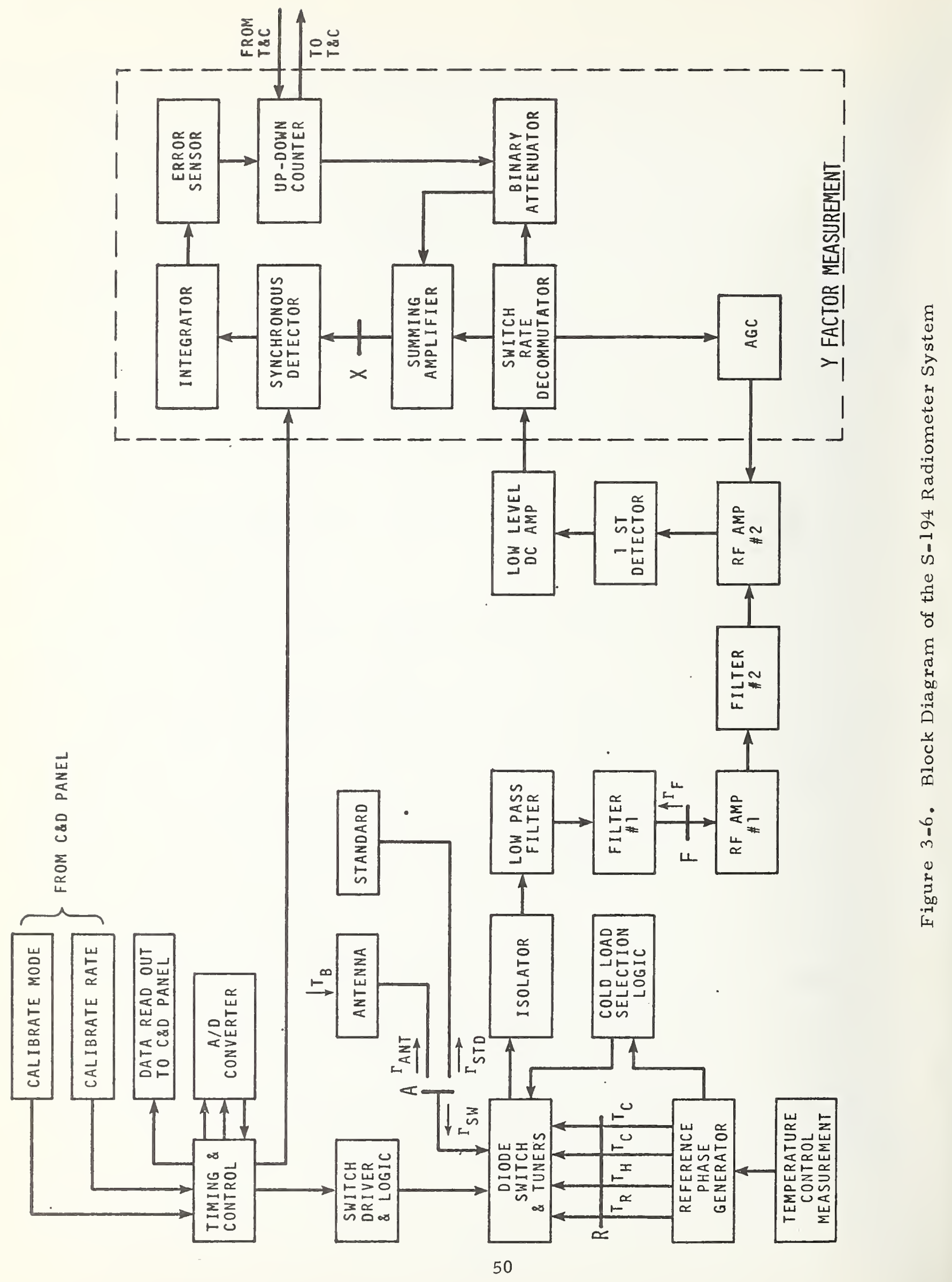


To evaluate the second term in (3-41) note that $\Theta_{F A}$ is the available power which originates in the front end of the radiometer between the reference planes A and F. It contains black-body radiation terms from the losses, leakage through the switch from the other switch ports, and shot noise generated by the switch. The shot noise contribution was measured by the contractor to be less than $1 \mathrm{~K}$, and the variation of this contribution with change of impedance cannot be corrected without further information. It is likely that the variation of this $1 \mathrm{~K}$ contribution with the radiometer input impedance is negligible, but tests to verify this would be worthwhile.

The isolation between switch ports insures that the leakage contribution to $\theta_{F A}$ is small, and variations due to input impedance should be negligible. The only remaining contribution to $\Theta_{F A}$ left to consider is the black-body radiation from the front-end losses. This contribution is [1]

$$
\theta_{F A}=\left(1-\alpha_{F A}\right) t_{D} \text {, }
$$

where $t_{D}$ is the physical temperature of the front end at the time of measurement. Thus (3-41) becomes

$$
\mathrm{T}_{0}^{\prime}=\mathrm{R} \mathrm{T}_{\mathrm{O}}-(1-\mathrm{R}) \mathrm{t}_{\mathrm{D}}+\left(\mathrm{T}_{\mathrm{AMP}}^{\prime}-\mathrm{T}_{\mathrm{AMP}}\right) / \alpha_{\mathrm{FA}}^{\prime} \cdot
$$

We are now in a position to express $T_{B}$ in terms of the parameters calibrated using the standard noise sources. Assuming that $\mathrm{T}_{\mathrm{B}}$ is constant over the main antenna beam, one finds from (2-9) and (2-11) that the power available from the antenna at A is

$$
\mathrm{T}_{\mathrm{A}}^{\prime}=\alpha^{\mathrm{MAIN}} \mathrm{T}_{\mathrm{B}}+\mathrm{T}_{\mathrm{ANT}} \text {, }
$$

where

$$
\mathrm{T}_{\mathrm{ANT}}=(1-\alpha) \mathrm{t}_{\mathrm{L}}+\mathrm{T}_{\mathrm{SIDE}},
$$


$\alpha$ is defined in (2-11), $\alpha^{\text {MAIN }}$ is obtained from (2-11) where the integral is only over the main beam, $t_{L}$ is the physical temperature of the antenna, and $\mathrm{T}_{\mathrm{SIDE}}$ is the radiation entering through the antenna side lobes available at A. The radiometer response to this available power is

$$
\mathrm{T}_{\mathrm{A}}^{\prime}=\mathrm{K}^{\prime} \mathrm{A}_{\mathrm{A}}-\mathrm{T}_{\mathrm{O}},
$$

where the primed parameters correspond to the antenna's impedance. Using $(3-40)$ and $(3-45)$,

$$
\mathrm{T}_{\mathrm{B}}=\left(1 / \alpha^{\mathrm{MAIN}}\right)\left\{\mathrm{R}\left(\mathrm{T}_{\mathrm{A}}-\mathrm{t}_{\mathrm{D}}\right)-\left(\mathrm{T}_{\mathrm{AMP}}^{\prime}-\mathrm{T}_{\mathrm{AMP}}\right) / \alpha_{\mathrm{FA}}^{\prime}+\left(\mathrm{t}_{\mathrm{D}}-\mathrm{T}_{\mathrm{ANT}}\right)\right\},
$$

where

$$
T_{A} \equiv K A_{A}-T_{0}
$$

The parameter $\mathrm{T}_{\mathrm{A}}$ is the temperature that a generator (having the same impedance as the calibration standards) would have in order to produce the output $A_{A}$. If the radiometer is calibrated with standards having the same impedance as the antenna, then $(3-49)$ simplifies to

$$
\mathrm{T}_{\mathrm{B}}=\left(\mathrm{T}_{\mathrm{A}}-\mathrm{T}_{\mathrm{ANT}}\right) / \alpha^{\mathrm{MAIN}} .
$$

\subsubsection{The Accuracy of the S-194 Radiometer}

To address the question of accuracy, we have a somewhat different view of the radiometer than the contractor. It seems to us that the two internal standards should be thought of as providing two reference noise amplitudes sent through the radiometer system to calibrate out any long-term drift in the output display words. The initial calibration using the external standards at $372 \mathrm{~K}$ and $80 \mathrm{~K}$, etc., is used to identify those brightness temperatures that correspond to these two reference amplitudes. The output of the radiometer is digital so that the brightness temperature of the radiation can vary over a range of about one degree before the output display word would change. In 
addition, however, the internal noise contributions can change over a one degree range before the output display word would be recalibrated. Thus the temperature that a particular output display word represents is in principle ambiguous over a two degree range. Ideally one would identify the center of this range for each output word so that the uncertainty would be plus or minus one degree.

Although the objective of the radiometer is to measure the brightness temperature $\mathrm{T}_{B}$, the errors in measuring $\mathrm{T}_{\mathrm{A}}$ (see Eqn. (3-49)) was given special attention by the contractor; and for comparison purposes, the errors in knowing $\mathrm{T}_{\mathrm{A}}$ will be discussed before continuing on to the problem of determining $\mathrm{T}_{B}$. Although the contractor defines $\mathrm{T}_{\mathrm{A}}$ (i.e. power available at plane A from a generator having the same impedance as the calibration standards), they analyze relative to $\mathrm{T}_{\mathrm{SA}}$, which is a delivered noise temperature, and thus is related via $T_{S A}=M_{A} T_{A}$ where

$$
\mathrm{M}_{\mathrm{A}} \equiv \frac{\left(1-\left|\Gamma_{\mathrm{ANT}}\right|^{2}\right)\left(1-\left|\Gamma_{\mathrm{SW}}\right|^{2}\right)}{\left|1-\Gamma_{\mathrm{ANT}} \Gamma_{\mathrm{SW}}\right|^{2}}
$$

where $\Gamma_{\text {ANT }}$ is the reflection coefficient looking toward the antenna, and $\Gamma_{S W}$ is the reflection coefficient looking toward the switch at the reference plane $A$. The mismatch factor $\mathrm{M}_{A}$ is about 0.81 for the $\mathrm{S}-194$, so errors in $\mathrm{T}_{\mathrm{SA}}$ and $\mathrm{T}_{\mathrm{A}}$ are comparable.

3.4.2.1 The Accuracy of $\mathrm{T}_{\mathrm{A}}$

The accuracy of determining $\mathrm{T}_{\mathrm{A}}$ depends on the resolution error ( $\pm 1 \mathrm{~K}$ al ready discussed), the calibration accuracy of the two reference amplitudes, linearity of the output, and errors in updating the reference amplitudes.

Calibration Error. The calibration by the contractor assumes that their hot and cold sources are $372 \pm 1 \mathrm{~K}$ and $81 \pm 1 \mathrm{~K}$, respectively, 
including a reduction in available power due to an adapter from the standard's connector to the radiometer's connector. After contacting the manufacturer of this standard, and because an unevaluated adapter was utilized, we estimate at least a $4 \mathrm{~K}$ error for the cold source and $2 \mathrm{~K}$ for the hot source. For assurance this adapter needs to be evaluated. This is to say that the standards which were used have not received an evaluation comparable to NBS noise standards that have about $\pm 1 \mathrm{~K}$ accuracy. Furthermore, because it is questionable that 81 Kelvins or 272 Kelvins are the most probable noise outputs, these errors should not be used in quadrature with other errors.

Using the standards $\mathrm{T}_{\mathrm{AH}}$ and $\mathrm{T}_{\mathrm{AC}}$ which give rise to the output display words $A_{A H}$ and $A_{A C}$, and using (3-31), the coefficients $K$ and $T_{0}$ are evaluated,

and

$$
K=\frac{T_{A H}-T_{A C}}{A_{A H}-A_{A C}},
$$

$$
T_{0}=\frac{A_{A C} T_{A H}-A_{A H} T_{A C}}{A_{A H}-A_{A C}} .
$$

Using these values for $K$ and $T_{0}$, the "calibration temperature" $\mathrm{T}_{\mathrm{AH}}^{*}$ and $\mathrm{T}_{\mathrm{AC}}^{*}$ are assigned to the output display words $\mathrm{A}_{\mathrm{AH}}$ and $\mathrm{A}_{\mathrm{AC}}$ produced by the two internal reference noise generators. The uncertainties in $\mathrm{T}_{\mathrm{AH}}^{*}$ and $\mathrm{T}_{\mathrm{AC}}^{*}$ are proportional to the uncertainties in $\mathrm{T}_{\mathrm{AH}}$ and $\mathrm{T}_{\mathrm{AC}}$ plus an additional error because this calibration procedure does not identify where within the one degree resolution zone the reference noise voltage is. Although the internal noise causes the digital output to oscillate between two states when amplitudes are close to a transition voltage, how close the reference voltages were to a particular transition voltage was not established. Thus the full one-degree resolution error should be added to the uncertainties in $\mathrm{T}_{\mathrm{AH}}^{*}$ and $\mathrm{T}_{\mathrm{AC}}^{*}$. Using $\mathrm{T}_{\mathrm{AC}}=81 \pm 4 \mathrm{~K}$, and $\mathrm{T}_{\mathrm{AH}}=372.2 \pm 2 \mathrm{~K}$, we conclude for the S-194 radiometer 


$$
\begin{aligned}
& \mathrm{T}_{\mathrm{AH}}^{*}=369.4 \pm 3 \mathrm{~K}, \\
& \mathrm{~T}_{\mathrm{AC}}^{*}=200 \pm 4.2 \mathrm{~K} .
\end{aligned}
$$

The resulting errors in $\mathrm{T}_{\mathrm{A}}$ due to calibration limitations are shown in Fig. 3-7. For readability, the errors are magnified by a factor of 10 . Thus, a display output of 650 implies that for $\mathrm{T}_{\mathrm{A}}=14 \mathrm{~K}$ a calibration error of $\pm 12 \mathrm{~K}$ occurs.

Linearity Error. Based on private communication with the test engineer, it is understood that linearity checks were made during an acceptance test for the radiometer over the range $90 \mathrm{~K}$ to $250 \mathrm{~K}$. Due to the accuracy of the attenuator utilized, it was possible to verify that deviations of less than $2 \mathrm{~K}$ occurred over the 90 to 250 range. It seems probable that the nonlinearity errors are due primarily to the uncertainty in the binary attenuator calibration. The error due to the binary attenuator was estimated to be on the order of $0.3 \mathrm{~K}$. A better linearity check needs to be performed in order to verify that the $0.3 \mathrm{~K}$ estimate is reasonable.

The equivalent $\mathrm{T}_{\mathrm{A}}$ 's corresponding to the two internal calibration outputs depend on the temperature of the internal standards, internal losses, and internal temperature distributions. The temperatures of the internal standards are monitored and are stabilized within $0.1^{\circ} \mathrm{K}$. This stabilization of the noise generators should also stabilize the associated loss distributions adequately. Thus no additional error is expected due to the minor temperature changes of the internal standards.

The calibration of the radiometer takes place with the coaxial transmission line between the antenna and radiometer switch at room temperature. In operation, the antenna will typically be at an ambient temperature near $190 \mathrm{~K}$. This results in a decrease of about $2 \mathrm{~K}$ for the thermal radiation from the cable losses for the operating situation compared with the 


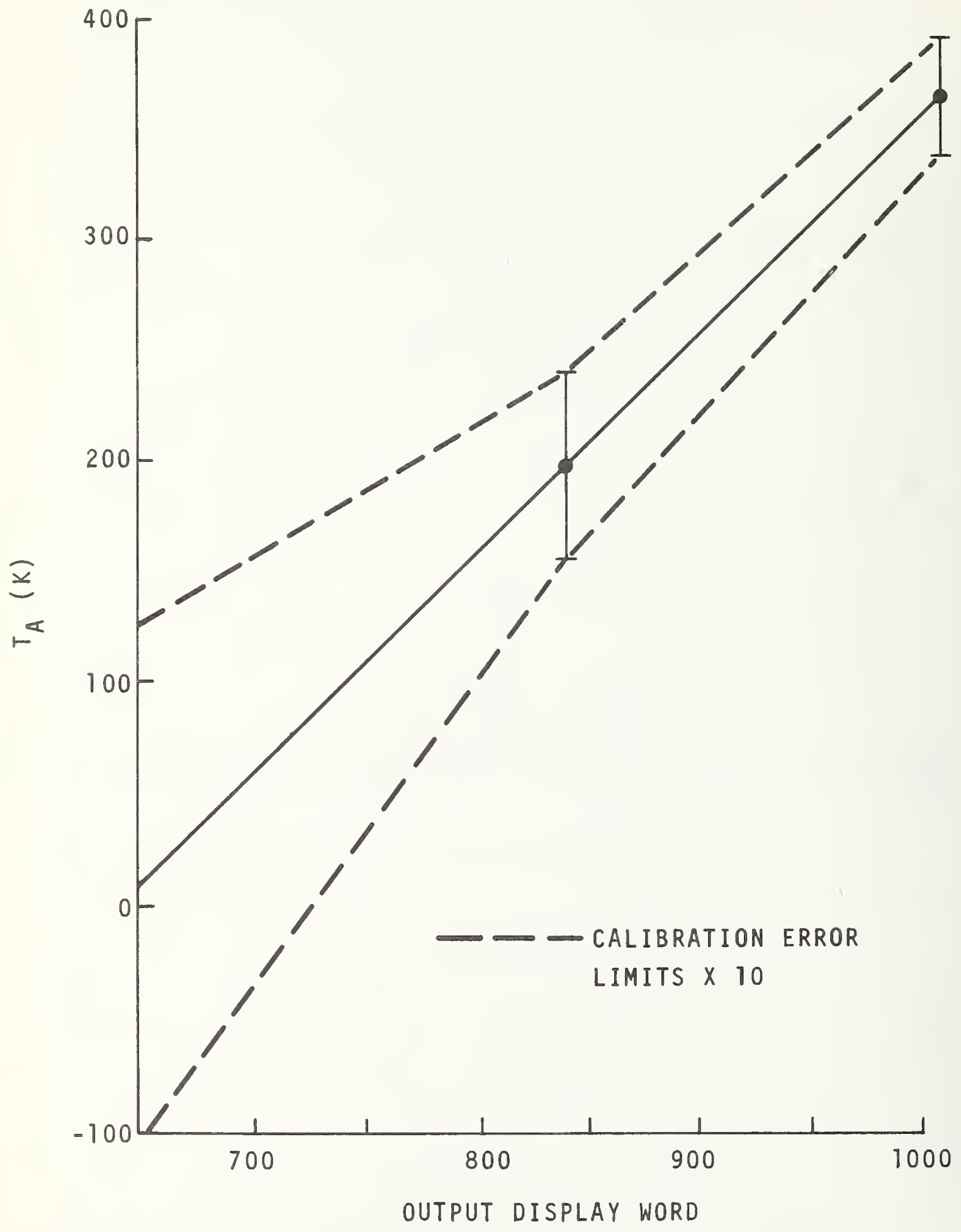
Figure 3-7. Effective Antenna Temperature $T_{A}$ vs Radiometer
Output with Calibration Error 
calibration situation. The contractor estimates a $25 \%$ uncertainty in the measurement of the cable loss, or a $\pm 0.5 \mathrm{~K}$ error in calculating the equivalent calibrated $\mathrm{T}_{\mathrm{A}}$ values. The contributions to the error in $\mathrm{T}_{\mathrm{A}}$ are summarized as follows.

$\begin{array}{ll}\text { Resolution } & \pm 1 \mathrm{~K} \\ \text { Calibration } & \pm 3 \text { at } 370 \mathrm{~K} \\ & \pm 4.2 \text { at } 200 \mathrm{~K} \\ & \pm 12.5 \text { at } 0 \mathrm{~K} \\ \text { Linearity } & \pm 0.3 \\ \text { Update } & \pm 0.5\end{array}$

The linearity, resolution, and update errors are probably in quadrature (i.e., independent, equally likely to be positive as negative, and more likely to be small than large). Thus the resultant linearity, resolution and update error is $\pm 1.2 \mathrm{~K}$. Our estimates for the errors in $\mathrm{T}_{\mathrm{A}}$ near $0 \mathrm{~K}, 200 \mathrm{~K}$ and $370 \mathrm{~K}$ are $\pm 13.7 \mathrm{~K}, \pm 5.4 \mathrm{~K}$ and $\pm 4.2 \mathrm{~K}$, respectively, compared with estimates of $\pm 1.7, \pm 0.9$, and \pm 1.3 , respectively, made by the contractor.

\subsubsection{The Accuracy of $\mathrm{T}_{\mathrm{B}}$}

From $\mathrm{T}_{\mathrm{A}}$ one can obtain $\mathrm{T}_{\mathrm{B}}$ using $(3-49)$. Additional errors occur because of uncertainty in $R$, because of the change in the amplifier noise temperature, $\mathrm{T}_{\mathrm{AMP}}$, and because of un-

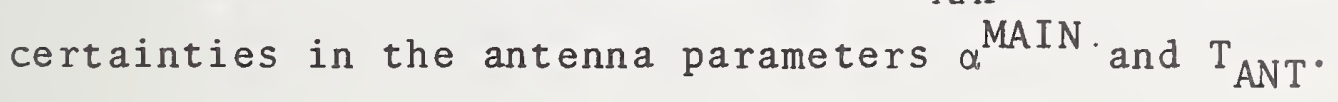

Accuracy of $R$. The parameter $R$ is given in (3-43). For the S-194 radiometer $\left|\Gamma_{\text {STD }}\right|$ was not measured but is probably less than $0.05,\left|\Gamma_{\text {ANT }}\right|=0.17 \pm 0.02,\left|\Gamma_{\text {SW }}\right|=0.025$, and $\left|\Gamma_{\text {SW }}\right|=$ 0.025 , and $\left|\Gamma_{F}\right|=0.017$. No phase information was obtained. It is estimated that $\left(1-\left|\Gamma_{F}^{\prime}\right|^{2}\right) /\left(1-\left|\Gamma_{F}\right|^{2}\right)=1 \pm 0.007$ so that

$$
R=1.03 \pm 0.027 \text {. }
$$


Change in Amplifier Noise Temperature. The change in $\left|\Gamma_{F}\right|$ of 0.01 as the antenna replaces the calibration noise source will change the amplifier's effective input noise temperature $T_{A M P}$ on the order of $0.4 \%$. Thus for a 4.1 dB noise figure amplifier, this implies

$$
\mathrm{T}_{\mathrm{AMP}}-\mathrm{T}_{\mathrm{AMP}}^{\prime} \simeq 1.8 \mathrm{~K}
$$

Uncertainties in Antenna Parameters. For the S-194 radiometer, we have estimated $\alpha=0.86 \pm 16 \%$ and $\alpha^{\text {MAIN }}=0.98 \alpha$. If we neglect uncertainties of the radiation entering the antenna via the side and back lobes, then from (3-47) and (3-49) the errors due to the uncertainties in the antenna loss and beam efficiency are about $0.16\left(\mathrm{~T}_{\mathrm{A}}-t_{L}\right)$ and $0.009 \mathrm{~T}_{\mathrm{B}}$, respectively.

Resultant Error in $T_{B}$. Using (3-49) and (3-56), the errors in $\overline{\mathrm{T}}_{\mathrm{B}}$ are shown in Table 3.5 , listed in order of their importance, for $T_{B}$ in the center of its expected range. Both the linear sum and the quadrature sum are 1isted. The appropriate error lies between these limits. Insofar as the sources of error are independent, and the most probable values for each parameter are used in the calculation, then the appropriate error will lie nearer the quadratic sum. In our opinion, the calibration error and the error due to a change in $\mathrm{T}_{\text {AMP }}$ do not satisfy the conditions for quadratic addition. The last entry in Table 3.5 lists the error if the calibration error and the error due to the changing $\mathrm{T}_{\text {AMP }}$ are added linearly, while the remaining errors are added quadratically.

\subsubsection{Comments}

The calibration procedure used by the contractor contributes a large error. In particular, for $\mathrm{T}_{\mathrm{B}}=175 \mathrm{~K}$, the use of an external standard to calibrate the radiometer contributed 
Table 3.5 Errors in Antenna Brightness Temperature For the Typical Conditions $t_{D}=297 \mathrm{~K}, t_{L}=190 \mathrm{~K}, \alpha^{\text {MAIN }}=0.84, \mathrm{~T}_{\mathrm{ANT}}=34 \mathrm{~K}$.

\begin{tabular}{|c|c|c|c|}
\hline $\begin{array}{c}\text { Parameter } \\
\text { Causing Error }\end{array}$ & $\begin{array}{c}\mathrm{T}_{\mathrm{B}}=0 \quad \mathrm{~K} \\
\left(\mathrm{~T}_{\mathrm{A}}=34 \mathrm{~K}\right)\end{array}$ & $\begin{array}{c}\mathrm{T}_{\mathrm{B}}=175 \mathrm{~K} \\
\left(\mathrm{~T}_{\mathrm{A}}=167 \mathrm{~K}\right)\end{array}$ & $\begin{array}{c}\mathrm{T}_{\mathrm{B}}=350 \mathrm{~K} \\
\left(\mathrm{~T}_{\mathrm{A}}=300 \mathrm{~K}\right)\end{array}$ \\
\hline Calibration & $\pm 13.7 \mathrm{~K}$ & $\pm 6.2 \mathrm{~K}$ & $\pm 1.9 \mathrm{~K}$ \\
\hline$\alpha$ & 25.0 & 3.7 & 17.6 \\
\hline Beam Efficiency & 0.0 & 1.6 & 3.1 \\
\hline $\mathrm{R}$ & 7.1 & 3.5 & 0.1 \\
\hline $\mathrm{T}_{\mathrm{AMP}}$ & 2.2 & 2.2 & 2.2 \\
\hline Resolution & 1.3 & 1.3 & 1.3 \\
\hline Update & 0.7 & 0.7 & 0.7 \\
\hline Linearity & 0.4 & 0.4 & 0.4 \\
\hline Linear Sum & $\pm 50.4 \mathrm{~K}$ & $\pm 20.0 \mathrm{~K}$ & $\pm 27.3 \mathrm{~K}$ \\
\hline Quadratic Sum & $\pm 29.5 \mathrm{~K}$ & $\pm 8.7 \mathrm{~K}$ & $\pm 18.2 \mathrm{~K}$ \\
\hline $\begin{array}{l}\text { Quad. except } \\
\text { Cal. and T AMP }\end{array}$ & $\pm 41.9 \mathrm{~K}$ & $\pm 13.9 \mathrm{~K}$ & $\pm 22.1 \mathrm{~K}$ \\
\hline
\end{tabular}

$\pm 6.8 \mathrm{~K}$ in the process of transferring the external calibration to the internal reference generators, $\pm 3.5 \mathrm{~K}$ to account for the errors due to the mismatch between the antenna and the radiometer versus the standard and the radiometer, and $\pm 2.2 \mathrm{~K}$ because the effective input noise temperature of the amplifier changes when the antenna is replaced by the standard. Thus $a \pm 12.5 \mathrm{~K}$ error component in the $\pm 14.3 \mathrm{~K}$ resultant error is due to using a standard with an impedance different than the antenna's.

If the loss measurement errors were $\pm 0.02 \mathrm{~dB}$ (as claimed), then these values could be used to calculate the reference temperature $\mathrm{T}_{\mathrm{AH}}^{*}$ and $\mathrm{T}_{\mathrm{AC}}^{*}$ to within $2 \mathrm{~K}$ instead of \pm 3 and \pm 4.2 as given in $(3-54)$ and $(3-55)$. Further, $(3-50)$ could be used instead of $(3-49)$ to eliminate errors due to $\mathrm{R}$ and $\mathrm{T}_{\text {AMP }}$. Under these conditions, the errors shown in Table 3.6 would hold and the quadrature sum should be valid. In case the old loss measurements are not trusted, a variable impedance standard 
adjusted to equal the antenna impedance might be used with comparable results. Matching the antenna impedance across the pass band of the amplifiers might not be practical so that some mismatch error would still remain. In any case, (3-49) should be adequate as a joining equation to use in estimating these errors. This is to say, (3-49) accounts for the multiple reflection and correlation terms which the contractor and Hartsfield were concerned about. It is also apparent from Table 3.5 that the antenna parameters need to be measured more accurately. Table 3.6 shows the uncertainties probably attainable by the use of improved antenna loss and beam efficiency measurements together with improved radiometer calibration, using either accurate loss measurements on the radiometer components or an adjustable impedance standard.

Table 3.6 Probable Attainable Errors in Antenna Brightness Temperature

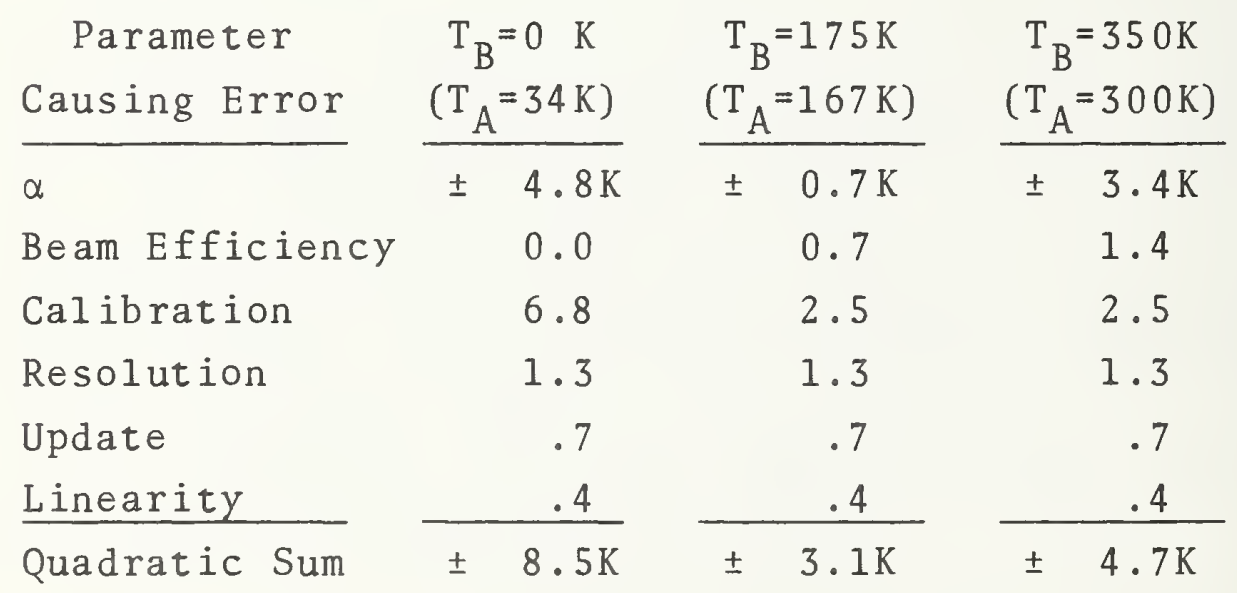




\subsection{Loss Measurements on the S-193 Antenna}

The antenna and its associated switching circuits are shown in figure 4-1. The numbered terminal ports are at important waveguide junctions and will be used in the following discussion.

There is a problem for this antenna when defining how much of the switching assembly should be considered a part of the antenna, and then making measurements at the appropriate junctions. Leakage and cross-talk within circulator $G$ affect the cross-polarization response of the antenna and, therefore, the gain and pattern measurements should definitely be taken at some terminal which includes $G$ as a port of the antenna system.

The output of circulator $G$ would be the best place to make these measurements; but it is not possible to get at this connection, so pattern measurements have been made at terminal IV. Gain measurements (to determine antenna efficiency) should also be made after circulator $G$, since the on-axis cross polarization is significant. If these gain measurements are made at terminal IV, they will include the efficiency of the antenna structure plus all of the waveguide switching assembly. However in operation, the switching assembly will be at a different temperature than the antenna, so the efficiency of each assembly must also be measured separately. The contractor was aware of this problem and therefore made pattern measurements at terminal IV, computed or measured the loss of the antenna to terminals II and III, measured the loss from terminals II and III to IV, and made gain measurements at terminals 0 and $0^{\prime}$. The pattern measurements have therefore included the effect of the first circulator, while the gain measurements have not. The antenna efficiency determined from the ratio 


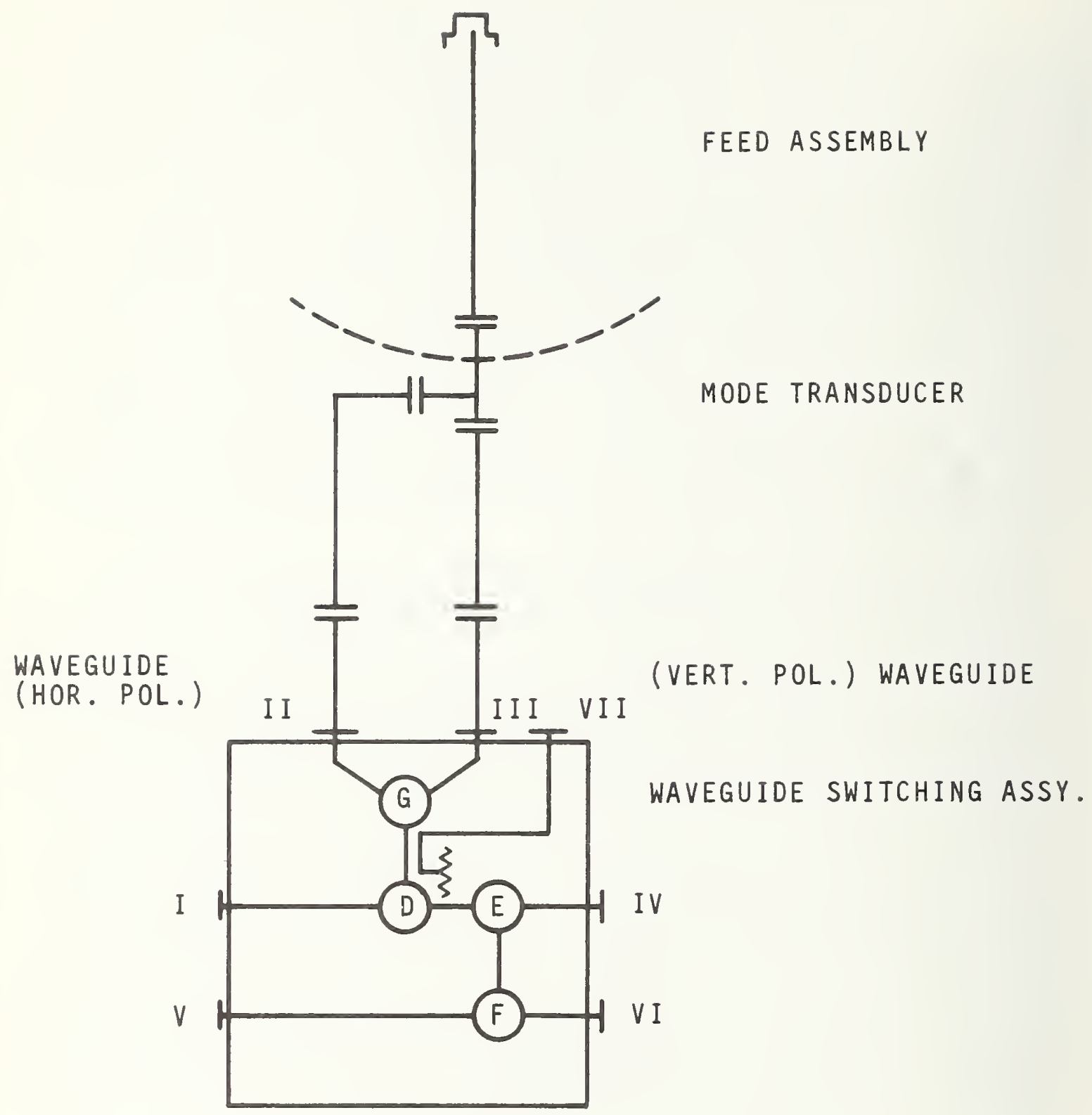

Figure 4-1. Schematic of S-193 Antenna and Switching Assembly . 
of gain to directivity will therefore have a small error due to this difference in measurement points.

Loss Computed from Antenna-Component Data. The contractor determined the loss of the antenna by measuring or calculating the loss in the individual components which make up the antenna input and feed assembly. These components are shown schematically in figure 4-2.

The components for both ports are composed of a 2 -inch straight section and a combination of bends of WR-62 waveguide plus the orthomode transducer. The losses of these components were measured in a reflectionless insertion-loss measuring system, and therefore attenuation rather than efficiency was determined. However, the loss was less than $0.20 \mathrm{~dB}$ in each case, so the difference between the two quantities is quite small. The other error in these measured attenuations is due to the inaccuracy of the measuring system. For the small losses measured, the error for the type of system used is about \pm 0.02 dB. The measured data and associated errors are listed below.

Table 4.1 Errors in Measured Efficiencies of Antenna Input Components

Measured Attenuation

Component

2 in. Straight Section

Vert. Port Bends

Hor. Port Bends

Orthomode Transducer Vert. Port

Orthomode Transducer Hor. Port
$(\mathrm{dB})$

$-.04$

$-.06$

$-.06$

$-.03$

$-.08$

System Efficiency vs. Error Attenuation Error

$(\mathrm{dB})$

$\pm .02$

$\pm .02$

$\pm .02$

$\pm .02$

$\pm .02$
$(\mathrm{dB})$

$+.02$

$+.02$

$+.02$

$+.01$

$+.03$

The errors, listed as efficiency vs, attenuation, were obtained by using the magnitudes of (a) the attenuation, (b) the 


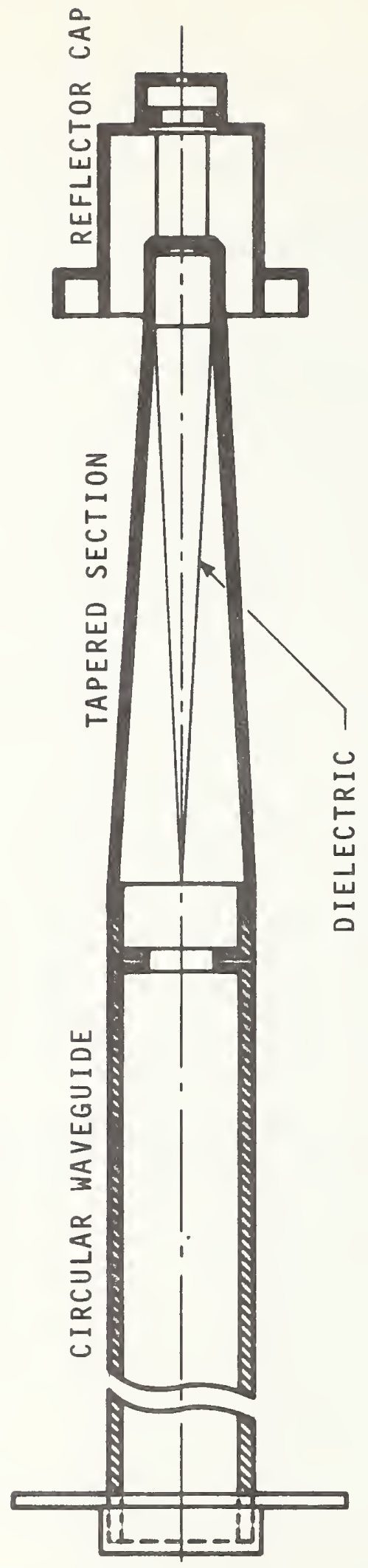

票 
input and output reflection coefficients for each section, and (c) the reflection coefficient of each preceding section as the source reflection coefficient $\Gamma_{\mathrm{g}}$. The efficiency (or $\alpha$ ) was then computed from equation (2-3) using all realizable phase combinations for the $\Gamma^{\prime} \mathrm{s}$.

The losses of the 12.920 inch long circular waveguide and the following tapered section with its tapered dielectric were determined by calculation. The calculation for the straight circular section is straightforward using formulas available in standard texts [6]. These calculations give a loss of $0.01 \mathrm{~dB}$ with an estimated uncertainty of $\pm 0.01 \mathrm{~dB}$. The tapered section geometry is approximated for purpose of calculation. It was assumed that the waveguide was of uniform circular cross section uniformly filled with the fused silica dielectric. The dielectric constant of the fused silica is 3.8 with a loss tangent of $2 \times 10^{-4}$. The calculated loss of this section using available formulas [7] is $0.16 \mathrm{~dB}$.

The contractor has added an additional $0.02 \mathrm{~dB}$ loss in the reflector cap and parabolic reflector. The total antenna loss is then tabulated below.

Table 4.2 Summary of Loss Components on S-193 Antenna

Horizontal Mode

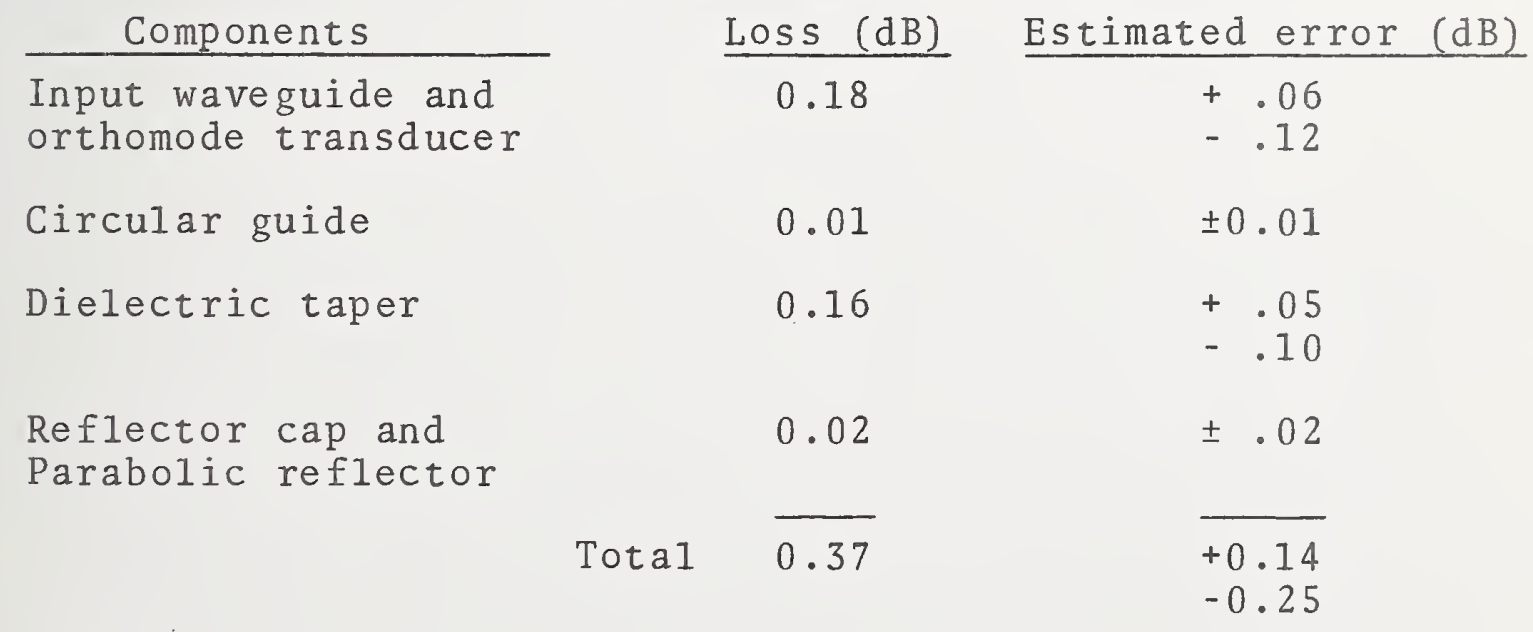


Vertical Mode

Components

Input waveguide and

orthomode transducer

Circular guide

Dielectric taper

Reflector cap and

Parabolic reflector
Loss $(d B)$

0.13

0.01

0.16

0.02

Total
Estimated Error (dB)

$+.06$

$-.12$

$\pm 0.01$

$+.05$

$-.10$

$\pm 0.02$

$+0.14$

$-0.25$

The total errors for the input waveguide and orthomode transducer combination are an arithmetic sum of the values in the first table. They were not added in quadrature since the system error would be the same for all three components, and the efficiency vs. attenuation errors are all positive.

For an incident brightness temperature of $\mathrm{T}_{\mathrm{B}}$ and an antenna temperature of $\mathrm{T}_{\mathrm{a}}$, the resulting error in antenna output noise temperature $\mathrm{dT}_{0}$ is given by,

$$
d T_{0}=\left(T_{B}-T_{a}\right) d \alpha
$$

For values of $\mathrm{d} \alpha$ of $+0.03,-0.06$ (which correspond to +0.14 and $-0.25 \mathrm{~dB}$, respectively), a temperature difference $\left(\mathrm{T}_{\mathrm{B}}-\mathrm{T}_{\mathrm{a}}\right)$ of 100 Kelvins would produce an error of +3 and -6 Kelvins, respectively.

Loss Measurements from Gain and Directivity. Although the contractor has not used the pattern data to compute directivity, it should be very simple to complete this calculation. This could then be used with the measured gain to compute the antenna efficiency and therefore obtain this parameter by two independent methods. This would serve as a check on both the loss measurements and the pattern measurements, and we recommend that this be done. 


\subsection{Gain Measurements on the S-193}

There was no documentation of the gain measurements available, and so the information and data referred to here have been obtained from discussion with the engineer who performed the measurements.

Two sets of measurements were made of the antenna gain. In the first set, the gain standard was connected to terminal I via a 12 -inch section of guide and the receiver was connected to terminal IV. Due to high wind conditions during the meas urement and other factors, the random error in this measurement was $\pm 0.4 \mathrm{~dB}$. Since a more accurate measurement was performed later, the first measurement will not be examined in detail.

The second gain measurement was done at the outputs of the orthomode transducer, ports 0 and $0^{\prime}$. The standard gain horn was mounted on the rotator pedestal so that it was pointing $90^{\circ}$ in azimuth away from the dish. When the horn was rotated into receiving position, it was at approximately the same location as one quadrant of the test antenna. Four readings were taken for each position of the horn, and it was then changed to another quadrant and four more readings taken. The resulting sixteen measurements were averaged to give one value for received power ratio which was used in the gain calculation.

Each power ratio measurement was made using the instrumentation shown in figure 4-3. The measurement procedure consisted of placing the test antenna in the receiving position, connecting the load to its output port, and adjusting the rotator for peak received signal. The gain of the receiver was then adjusted for a zero $d B$ reading on the SWR meter. The standard gain horn was then rotated into position, and the load connected to its output port. The rotator was again positioned for maximum received signal, and the change in signal level was read on the SWR meter. The test antenna was rotated back into receiving 


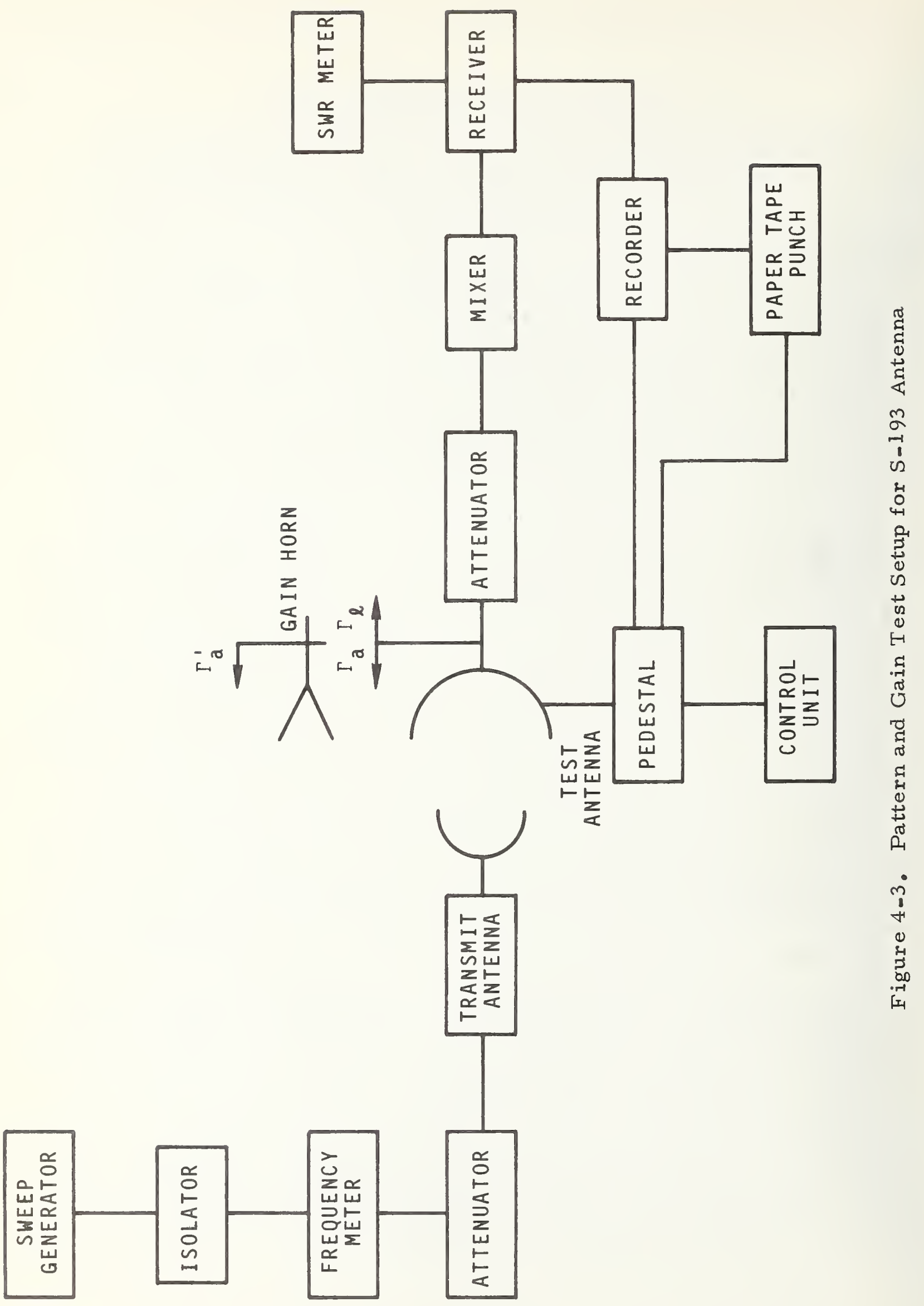


position, the load replaced on its output port, and the SWR meter read again. Values of power ratio were therefore obtained going from test to standard and from standard to test, and these two values were averaged to reduce the effect of drift and random error.

The data obtained from these measurements are:

$f=13.9 \mathrm{GHz}$

Gain of Standard $=10 \log \mathrm{G}^{\prime}=24.10 \mathrm{~dB}$

Power ratio (average of 16 ) $=1010 \mathrm{~g} \mathrm{P}_{\mathrm{d}} / \mathrm{P}_{\mathrm{d}}^{\prime}=17.0 \mathrm{~dB}$ Separation distance, $\mathrm{d}=\mathrm{d}^{\prime}=1432 \mathrm{~m}$.

$$
\begin{aligned}
& \left|\Gamma_{\mathrm{a}}\right|=0.05 \\
& \left|\Gamma_{\mathrm{a}}^{\prime}\right|=0.03 \text { to } 0.05 \\
& \left|\Gamma_{\ell}\right|=0.05 \\
& \left|\rho_{\mathrm{T}}\right|=0.01 \text { to } 0.03 \\
& \left|\rho_{\mathrm{S}}\right|=0.01 \text { to } 0.03 \\
& |\rho|=0.08 \text { to } 0.10
\end{aligned}
$$

Since the same load circuit, consisting of the output attenuator and mixer, was used with each antenna, and no waveguide was used between the antennas and the load, equation (3-17) becomes,

$$
\frac{P_{d}}{P_{d}^{\prime}}=\frac{\left(1-\left|\Gamma_{a}\right|^{2}\right)\left|1-\Gamma_{a}^{\prime} \Gamma_{\ell}\right|^{2}\left|1-\rho_{T}\right|^{2}\left(1+\left|\rho^{\prime}\right|^{2}\right) d^{\prime 2} G}{\left(1-\left|\Gamma_{g}^{\prime}\right|^{2}\right)\left|1-\Gamma_{a} \Gamma_{\ell}\right|^{2}\left|1-\rho_{T^{\prime}} \rho^{\prime}\right|^{2}\left(1+|\rho|^{2}\right) d^{2} G^{\prime}}
$$

The sources of error in determining $G$ are then as follows: 1. Error in Measuring Power Ratio. The manufacturer specifies the error in the receiver to be $\pm 0.25 \mathrm{~dB}$. This is also about the error which would result if the uncalibrated attenuators were used to measure the power ratio.

2. Gain Standard Error. Since an uncalibrated gain standard was used, its error is estimated to be $\pm 0.25 \mathrm{~dB}$. 
3. Distance Error. The long-range distance used makes probable distance errors insignificant.

4. Transmission Line Mismatch. If the amplitude and phase of each reflection coefficient were known, a complete correction could be made for the mismatch. Since only the magnitudes are known, we can estimate a worst case error of \pm 0.04 $\mathrm{dB}$ due to mismatch.

5. Polarization Mismatch. The source antenna and standard gain horn have axial ratios of 30 to $40 \mathrm{~dB}$. Due to the crosstalk in the first circulator, the axial ratio of the test antenna is only $20 \mathrm{~dB}$. This combination of polarization ratios would produce an error of $0.06 \mathrm{~dB}$.

6. Unequal $\alpha$ 's for the Transmission Line. This error does not apply for this measurement because $\alpha$ was the same for both antennas.

7. Multipath Error. The multipath effect is reduced by moving the horn to the four different positions and averaging the results. A residual multipath error of $\pm 0.05 \mathrm{~dB}$ is probable.

8. Near-Zone Error. The very long-range distances again make this error negligible.

9. Random Error. From the sixteen measured power ratios for one gain computation the random error was $\pm 0.05 \mathrm{~dB}$. 10. Ground Reflection Error. In the comparison technique this error occurs only if the two antennas have significantly different patterns. It was considered negligible here.

The resultant effect of these errors is summarized in Table 4.3. This error could be reduced without further measurement on the S-193 antenna by calibrating the gain standard and the detector-receiver used to measure the power ratio. If this were done, the gain error could be reduced to about $\pm 0.15 \mathrm{~dB}$. 
Table 4.3 Summary of Errors in Gain Measurements.

Source of Error

(1) Power Ratio Measurement

(2) Standard Gain Horn Gain

(3) Distance Measurement

(4) Transmission Line Mismatch

(5) Polarization Mismatch

(6) Unequal $\alpha$ 's

(7) Multipath Signals

(8) Near-Zone

(9) Random Error
Error in Gain (dB)

$\pm 0.25$

$\pm 0.25$

$\pm 0.00$

$\pm 0.04$

$\pm 0.06$

$\pm 0.00$

$\pm 0.05$

$\pm 0.00$

RSS $=\frac{ \pm 0.05}{ \pm 0.37 \mathrm{~dB}}$ 
4.3 Pattern Measurement, Beam Efficiency, and Directivity of the S-193 Antenna

\subsubsection{Introduction}

The purpose of the pattern measurement is to determine the beam efficiency and directivity, and to use the directivity with the previously measured gain to determine the antenna efficiency (loss).

The following notation will be used in this section to refer to measured and computed parameters.

$$
\begin{aligned}
G= & \text { antenna gain in the boresight direction. } \\
\mathrm{D}= & \text { antenna directivity in the boresight direction. } \\
\Phi^{\prime}(\alpha, \beta)= & \text { radiation intensity (power per unit solid } \\
& \text { angle) transmitted by the antenna in the direc- } \\
& \text { tion defined by the angles } \alpha \text { and } \beta . \\
\Phi_{O}^{\prime}= & \text { radiation intensity in the boresight direction. } \\
\mathrm{P}_{\mathrm{T}}^{\prime}= & \text { total power radiated by antenna. } \\
\mathrm{P}_{\mathrm{m}}^{\prime}= & \text { power radiated in main beam. } \\
\mathrm{P}_{\mathrm{S}}^{\prime}= & \text { power radiated in all side and back lobes. } \\
\mathrm{P}_{\mathrm{i}}^{\prime}= & \text { net power delivered to antenna input. } \\
\varepsilon= & \text { antenna beam efficiency. } \\
n= & \text { antenna efficiency (loss). } \\
\Omega= & \text { solid angle. } \\
\mathrm{AZ}= & \text { rotator mount aximuth angle. } \\
\mathrm{EL}= & \text { rotator mount elevation angle. }
\end{aligned}
$$

Since only relative pattern measurements are made and required to compute beam efficiency and directivity, we may use relative rather than absolute values for the above power quantities. Using the radiation intensity in the boresight direction as the normalization factor, and denoting the absolute quantities by primes, the relative quantities are 


$$
\begin{aligned}
\Phi(\alpha, \beta) & =\frac{\Phi^{\prime}(\alpha, \beta)}{\Phi_{0}^{\prime}}, \quad \Phi_{0}=\frac{\Phi_{0}^{\prime}}{\Phi_{0}^{\prime}} \equiv 1, \\
P_{T} & =\frac{P_{T}^{\prime}}{\Phi_{0}^{\prime}}, \quad P_{m}=\frac{P_{m}^{\prime}}{\Phi_{0}^{\prime}}, \quad P_{S}=\frac{P_{S}^{\prime}}{\Phi_{0}^{\prime}}, \\
P_{i} & =\frac{P_{i}^{\prime} .}{\Phi_{0}^{\prime}}
\end{aligned}
$$

The $\Phi$ quantities are dimensionless, and the normalized powers have the units of steradians.

In terms of the above quantities, the following relations exist.

$$
\begin{aligned}
& G=\frac{4 \pi \Phi_{0}^{\prime}}{P_{i}^{\prime}}=\frac{4 \pi}{P_{i}}, D=\frac{4 \pi \Phi_{O}^{\prime}}{P_{T}^{\prime}}=\frac{4 \pi}{P_{T}}, \\
& \eta=\frac{P_{T}}{P_{i}}=\frac{G}{D}, \varepsilon=\frac{P_{m}}{P_{T}}=\frac{P_{m}}{P_{m}+P_{S}}, \\
& P_{m}=\varepsilon P_{T}, P_{S}=(1-\varepsilon) P_{T}, P_{T}=P_{m}+P_{S} .
\end{aligned}
$$

The errors in $\varepsilon$ and $D$ due to the errors in the two partial powers are

$$
\begin{aligned}
& \frac{\mathrm{d} \varepsilon}{\varepsilon}=\frac{(1-\varepsilon)}{\varepsilon \mathrm{P}_{\mathrm{T}}} \mathrm{dP} \mathrm{m}_{\mathrm{m}}-\frac{1}{\mathrm{P}_{\mathrm{T}}} \mathrm{dP} \mathrm{P}_{\mathrm{S}}=(1-\varepsilon)\left(\frac{\mathrm{dP} \mathrm{m}_{\mathrm{m}}}{\mathrm{P}_{\mathrm{m}}}-\frac{\mathrm{dP} \mathrm{S}}{\mathrm{P}_{\mathrm{S}}}\right\}, \\
& \frac{d D}{D}=-\frac{d P_{m}}{P_{T}}-\frac{d P_{S}}{P_{T}}=-\left(\varepsilon \frac{d P_{m}}{P_{m}}+(1-\varepsilon) \frac{d P_{S}}{P_{S}}\right) \text {. }
\end{aligned}
$$

For antennas with $\varepsilon>0.90$, the error in side 1 obe power has 1ittle effect on the directivity, and the effect of errors in both partial powers on the beam efficiency is reduced by the factor $(1-\varepsilon)$. 


\subsubsection{General Measurement Approach}

The first step in the measurement is to define a set of coordinate axes which are fixed in the antenna. The vector components of the E-field are then defined with respect to these coordinates, and the scheme used to measure these components must be consistent with the chosen coordinate system. In other words, the axes and angles defined in the antenna coordinate system will be appropriate for a specific type of rotator (AZ/EL, EL/AZ or model mount) and orientation of the antenna on the rotator. The same angles will probably not be appropriate if the type of rotator or antenna orientation on the rotator is changed.

Let us define two coordinate systems, one fixed in space and denoted by $O \bar{X} \bar{Y} \bar{Z}$, and one fixed in the antenna and denoted by oxyz. The $\bar{Y}$ and $\bar{Z}$ - axes of the space system are shown in figure 4-4. The $\bar{X}$-axis is coincident with the elevation axis of the azimuth/elevation rotator used by the contractor. If the antenna is to be placed on the rotator as shown in figure 4-4, then the azimuth rotation will always rotate the antenna about its own $y$-axis (except for a slight parallaxerror). One angle in the antenna coordinate system should therefore be defined as a rotation about the y-axis.

The angle $\alpha$ shown in figure $4-5$ is this angle. The angle $\beta$ is defined as a rotation about the $x^{\prime}$ axis (which becomes the $x$-axis for $\alpha=0$ ). Every direction of propagation denoted by the propagation vector $\underline{k}(\alpha, \beta)$ relative to the antenna is defined by these two angles $\alpha$ and $\beta$ through the relations,

$$
\begin{array}{lll}
k_{x}=|\underline{k}| \cos \beta \sin \alpha & \\
k_{y}=|\underline{k}| \sin \beta & |\underline{k}|=\frac{2 \pi}{\lambda} \\
k_{z}=|\underline{k}| \cos \beta \cos \alpha . &
\end{array}
$$

At a large distance from the antenna, the total electric field transmitted by the test antenna is contained in the plane 


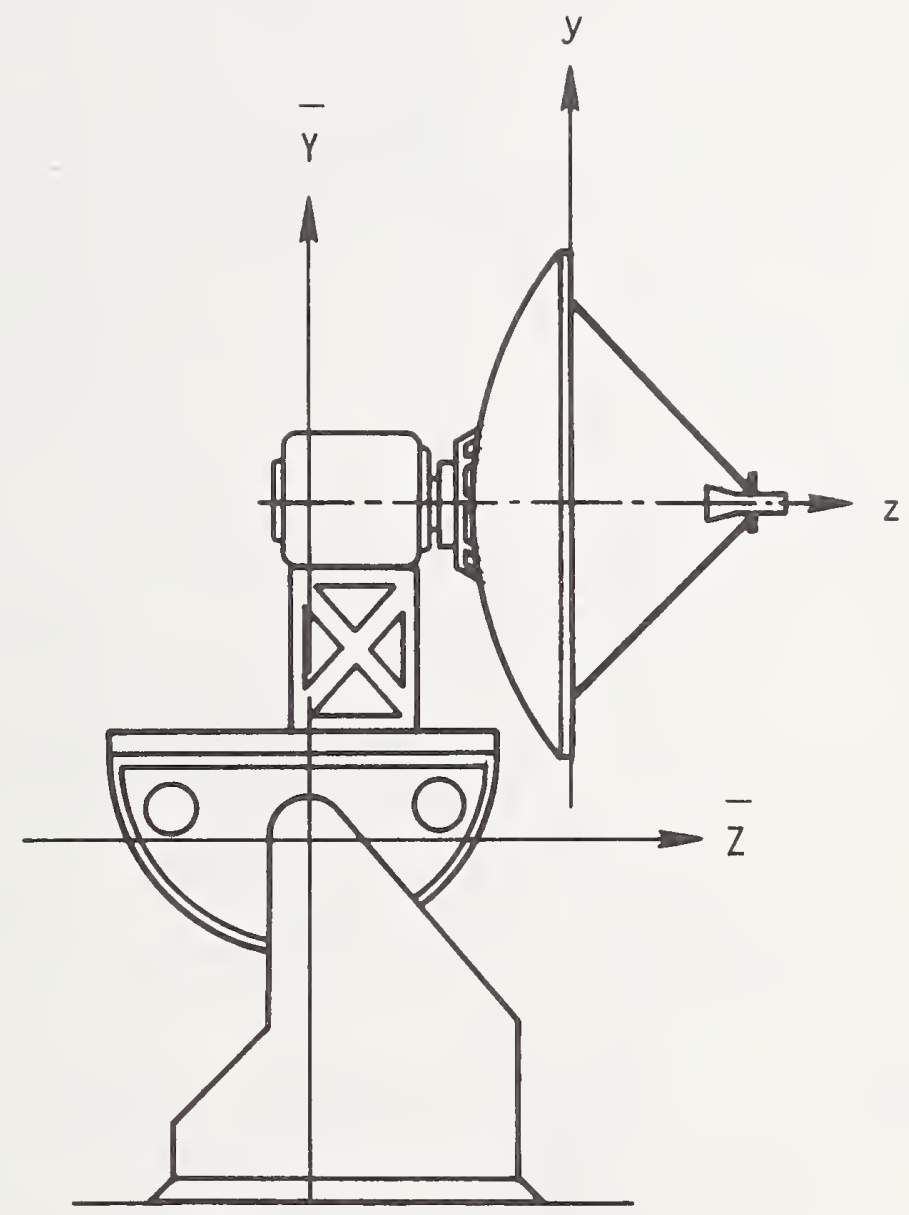

Figure 4-4. Rotator with Space and Antenna Coordinate Systems 


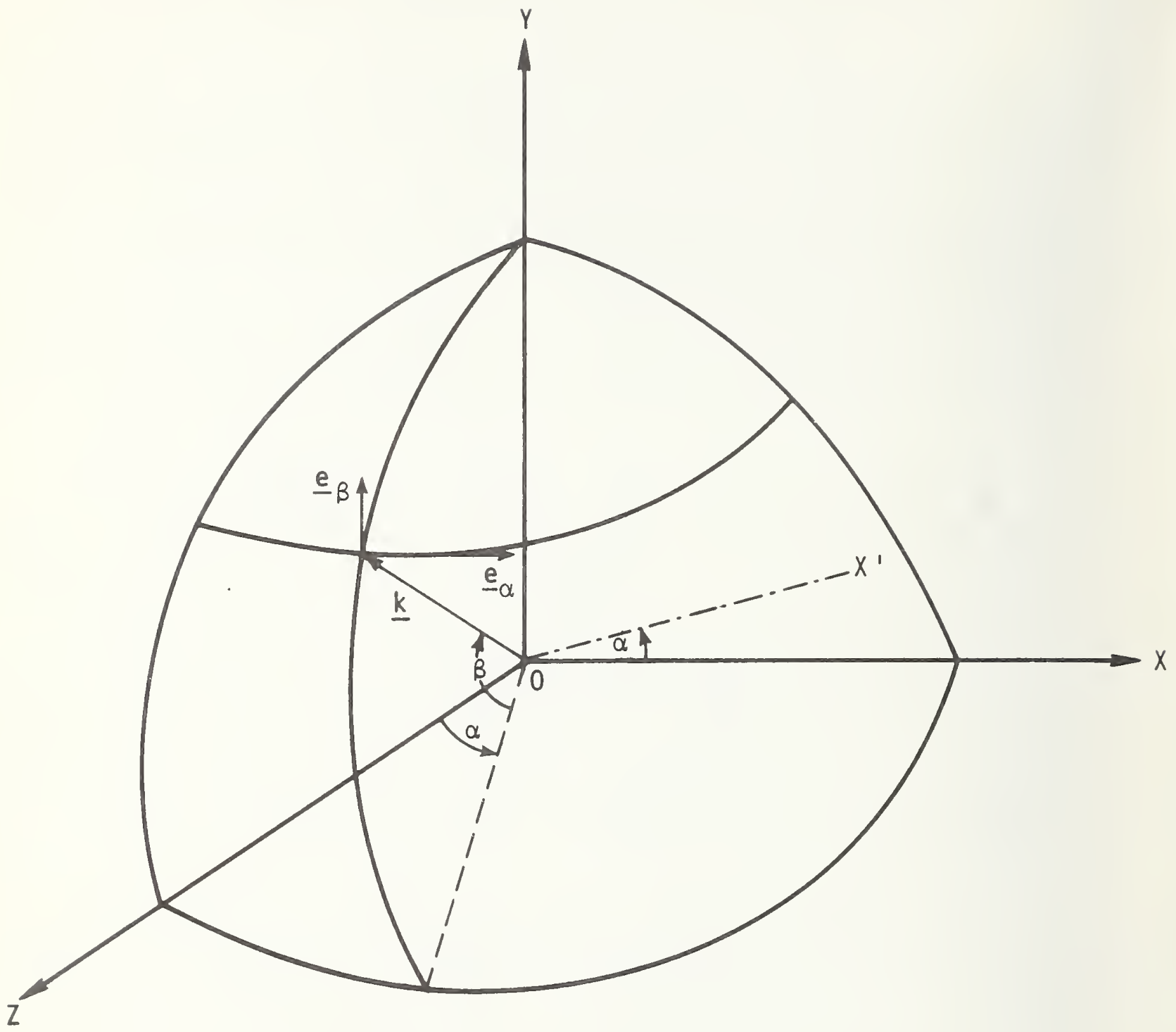

Figure 4-5. $\alpha-\beta$ Coordinate System Used with the S-193 Antenna 
of $\underline{e}_{\alpha}$ and $\underline{e}_{\beta}$, and the components of $\underline{E}$ will be denoted by $E_{\alpha}(\alpha, \beta)$ and $E_{\beta}(\alpha, \beta)$. The unit vectors $\underline{e}_{\alpha}$ and $\underline{e}_{\beta}$ are related to the unit vectors in the $x, y$ and $z$-directions by the relations,

$$
\begin{aligned}
& \underline{e}_{\alpha}=\cos \alpha \underline{e}_{x}-\sin \alpha \underline{e}_{z} \\
& \underline{e}_{\beta}=-\sin \alpha \sin \beta \underline{e}_{x}+\cos \beta \underline{e}_{y}-\cos \alpha \sin \beta \underline{e}_{z} .
\end{aligned}
$$

If the antenna is placed on the rotator as shown in figure 4-4 and the rotator moved through the angles, azimuth $=-\alpha$, elevation $=-\beta$, it is evident from figure 4-5 that the vector $k(\alpha, \beta)$ will be coincident with the space $\bar{Z}$-axis; $\underline{e}_{\beta}$ will be coincident with the space $\bar{Y}$-axis; and $\underline{e}_{\alpha}$ will be coincident with the space $\bar{X}$-axis. If we let $S_{\bar{X}}$ and $S_{\bar{Y}}$ denote the far-field on-axis $\bar{X}$ - and $\bar{Y}$-components of the E-field from the source antenna, then the signal received by the test antenna will be given by

$$
\begin{aligned}
b_{1}(-\alpha,-\beta) & =C\left[S_{\bar{Y}} E_{\beta}(\alpha, \beta)-S_{\bar{X}} E_{\alpha}(\alpha, \beta)\right] \\
& =C^{\prime} E_{\beta}(\alpha, \beta)\left[1-\rho_{S} \rho_{T}(\alpha, \beta)\right],
\end{aligned}
$$

where $\rho_{\mathrm{T}}$ and $\rho_{\mathrm{S}}$ are complex polarization factors given by

$$
\rho_{S}=\frac{S_{\bar{X}}}{S_{\bar{Y}}}, \quad \rho_{T}(\alpha, \beta)=\frac{E_{\alpha}(\alpha, \beta)}{E_{\beta}(\alpha, \beta)},
$$

$C$ is a constant for fixed separation distance, and $C^{\prime}=\operatorname{CS}_{\bar{Y}}$.

If either polarization factor is zero, then the output signal is proportional to the $\beta$-component of the electric field in the direction defined by $\alpha$ and $\beta$. Since in most cases neither factor is zero, the term, $1-\rho_{S} \rho_{T}(\alpha, \beta)$, represents an error in the measurement of $E_{\beta}$ due to cross polarization. 
Since only relative power patterns are to be measured, $b_{1}(-\alpha,-\beta)$ is normalized by the power in the boresight direction, giving

$$
B_{1}(-\alpha,-\beta)=\left|\frac{b_{1}(-\alpha,-\beta)}{b_{1}(0,0)}\right|^{2}=\frac{\left|E_{\beta}(\alpha, \beta)\left[1-\rho_{S} \rho_{T}(\alpha, \beta)\right]\right|^{2}}{\left|E_{\beta}(0,0)\left[1-\rho_{S} \rho_{T}(0,0)\right]\right|^{2}}
$$

Measurement of $B_{1}$ for $\alpha=0$ to $360^{\circ}$ and $\beta=+90^{\circ}$ to $-90^{\circ}$ can yield complete relative power patterns for the $\beta$ component of the field if the polarization factor is unity.

If the source antenna is now rotated about its axis by $90^{\circ}$, the normalized received signal will be given by

$$
B_{2}(-\alpha,-\beta)=\frac{\left|E_{\alpha}(\alpha, \beta)\left[1+\rho_{S} / \rho_{T}(\alpha, \beta)\right]\right|^{2}}{\left|E_{\beta}(0,0)\left[1-\rho_{S} \rho_{T}(0,0)\right]\right|^{2}}
$$

Measurement of $B_{2}$ for the same range of angles as $B_{1}$ will give complete relative power patterns for the $\alpha$-component of the electric field. These will also be in error due to the polarization error factor unless the amplitudes and phases of $\rho_{S}$ and $\rho_{T}(\alpha, \beta)$ are known.

In the following discussion, the polarization error factors in (4-16) and (4-17) will be neglected. This is what is usually done, and what the contractor did in determining the radiated powers from the measured data. The error caused by this assumption will then be evaluated in the section on errors.

The total and main beam powers contained in the $\beta$ component of the field are then given by

and

$$
\mathrm{P}_{\mathrm{T} \beta}=\int_{0}^{2 \pi} \int_{-\pi / 2}^{\pi / 2} \mathrm{~B}_{1}(\alpha, \beta) \cos \beta \mathrm{d} \beta \mathrm{d} \alpha,
$$

$$
\mathrm{P}_{\mathrm{m} \beta}=\int_{\substack{\text { main } \\ \text { beam }}} \mathrm{s}_{1}(\alpha, \beta) \cos \beta \mathrm{d} \beta \mathrm{d} \alpha,
$$


with similar relations for the $\alpha$-component.

Since measurements are made at discrete points, the integration is carried out by an approximate numerical technique such as the trapezoidal rule. If we assume that $B_{1}$ was measured at uniform spacings in the $\alpha$ and $\beta$ directions given by

$$
\Delta \alpha=\frac{2 \pi}{M}, \quad \alpha_{i}=i \Delta \alpha, \quad \Delta \beta=\frac{\pi}{N}, \quad \beta_{j}=j \Delta \beta,
$$

then the total power is given by,

$$
P_{T \beta}=\frac{2 \pi^{2}}{N M} \sum_{j=1}^{N} \sum_{i=1}^{M} B_{1}\left(\alpha_{i}, \beta_{i}\right) \cos \beta_{i} .
$$

\subsubsection{Measurement Procedure used by the Contractor}

The last section has briefly described the steps which should be followed to obtain beam efficiency and directivity from measured power patterns. The actual procedure used by the contractor on the S-193 antenna is a modification of the described procedure, which in some cases has introduced errors into the results. The steps in the measurement procedure will now be described for the case of the vertical output port. The case for the horizontal port is similar. These measurement procedures and some of the data have been obtained primarily from discussion with the engineer who performed them. There is very little documentation of the measurement procedures.

The coordinate system definition for the antenna is such that the $y$-axis is in the direction of the principal component of the electric field in the boresight direction. The z-axis is along the boresight direction, and the $x$-axis is orthogonal to $y$ and $z$. The angles $\alpha$ and $\beta$ are defined as in figures $4-4$ and 4-5 with the $y$-axis as the $\alpha$-axis.

For future reference, it will be helpful to represent the range of directions defined by $\alpha$ and $\beta$ in a rectangular coordinate system, with $\alpha$ ranging between $-\pi$ and $\pi$, and $\beta$ 
ranging between $-\pi / 2$ and $\pi / 2$ as shown in figure 4-6. The numbering of the quadrants will be used to identify the regions over which digital and analogue data were taken. This designation of direction space by a quadrant number is the same as was used by the contractor in their reports. It must be kept in mind that this diagram represents directions with respect to the antenna coordinate system and not the space system. Therefore, to measure the patterns in quadrants 2 and 3 which correspond to negative values for $\beta$ and $k_{y}$, without reorienting the antenna on the mount, the elevation angle of the rotator mount is positive.

Table 4.4 Elevation Increments in Pattern Measurements

Elevation Angles

$$
\begin{aligned}
& 0^{\circ}-5^{\circ} \\
& 6^{\circ}-10^{\circ} \\
& 10^{\circ}-20^{\circ} \\
& 20^{\circ}-80^{\circ}
\end{aligned}
$$

Elevation Angle Increment

$0.5^{\circ}$

$2.0^{\circ}$

$5.0^{\circ}$

$10.0^{\circ}$

The antenna was placed on the azimuth/elevation rotator as shown in figure 4-4 such that for the azimuth and elevation angles both equal to zero, the space and antenna coordinate systems were coincident. (Actually due to physical limitations, the origins cannot be made coincident, and corresponding axes of the two systems are only parallel.)

Due to large ground reflections when the antenna was pointed towards the ground (negative elevation angles), reliable data could only be taken for positive elevation angles. There were also significant reflections on one side of the range, and this limited the motion of the mount to positive (counter clockwise) azimuth angles. The result of these two restrictions was that digital data were taken for only one quadrant of the mount's total motion without reorientation of the antenna on the mount. With the antenna oriented as in figure 4-4, rotation of the mount in positive azimuth and elevation angles 


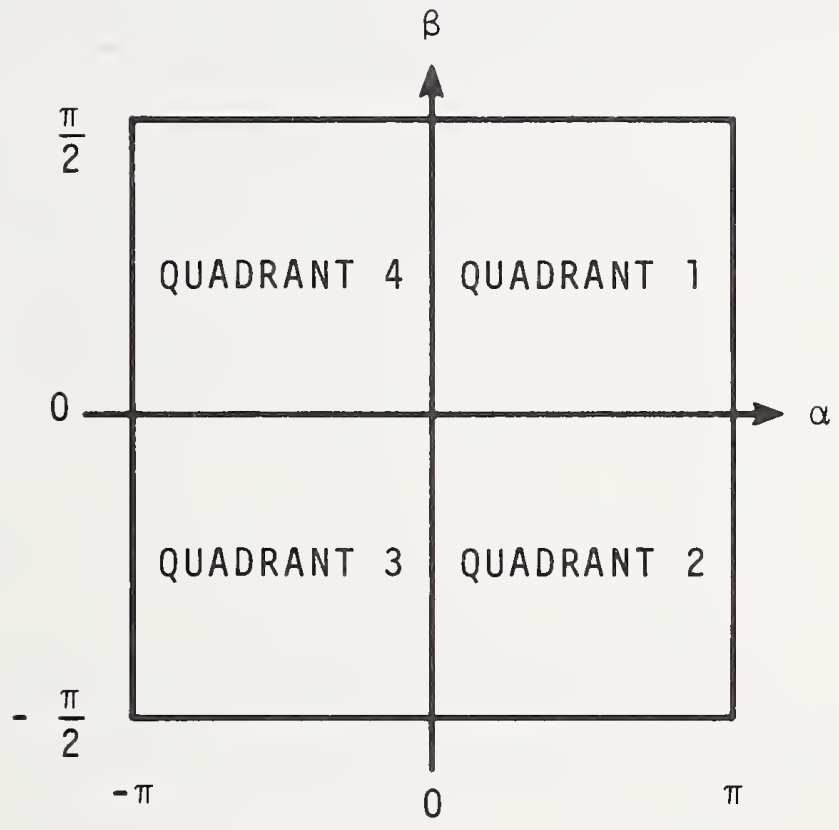

Figure 4-6. $\alpha-\beta$ Direction Space and the Four Measurement Quadrants 
produced data only in quadrant 3. The elevation angle was incremented and cuts taken along lines of constant elevation. The increments varied with the distance from boresight as shown in Table 4.4 and in figure 4-7. In addition to the digital data taken in quadrant 3 , analogue data were taken for both polarizations in quadrants 3 and 2 . These data were taken at the same time as the digital data but extended $30^{\circ}$ into quadrant 2 for each elevation step. On the basis of the analogue data it was assumed that the patterns were identical (or at least that the total powers were identical) in quadrants 3 and 2 .

Figures 4-8 and 4-9 are samples of the analogue plots in quadrants 3 and 2. From these and similar plots contained in the "Calibration Data Report, Flight Hardware, Volume 11," it appears that the patterns are quite symmetrical between 3 and 2. For amplitudes down to about $-30 \mathrm{~dB}$ the differences are only a few tenths of $a d B$, and there is general agreement between $-30 \mathrm{~dB}$ and $-50 \mathrm{~dB}$. Thus it would appear that the assumption of 3-2 symmetry is fairly good.

No analogue plots or digital data have been available to justify an assumption of symmetry between quadrants 1 and 4, and yet this is implicit in the approach which was made. Perhaps some analogue plots were taken which showed the same basic characteristics as those in figures 4-8 and 4-9, or else the 3-2 symmetry was assumed sufficient to justify 4-1 symmetry. Whatever the reason, we do not have sufficient information to evaluate this assumption.

With the assumption of 3-2 and 4-1 symmetry, and data in quadrant 3 , the total power pattern can be obtained by either justifying 2-1 and 3-4 symmetry or obtaining digital data in quadrants 1 or 4 .

With the antenna in its initial orientation on the rotator, as shown in figure 4-4, pattern measurements in quadrants 1 or 4 would require pointing the antenna towards 

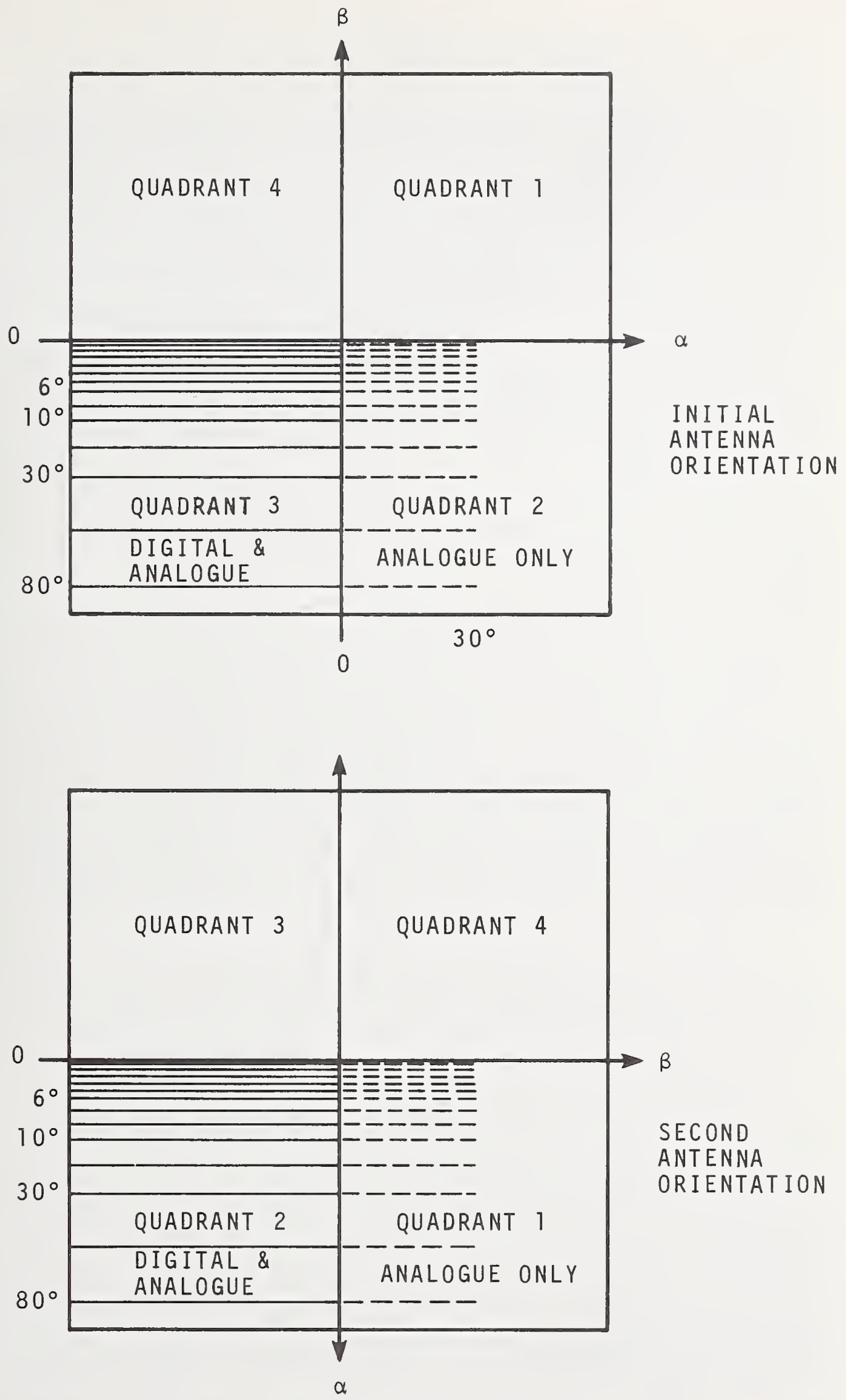

Figure 4-7. Measurement Schemes for the Two Antenna Orientations 

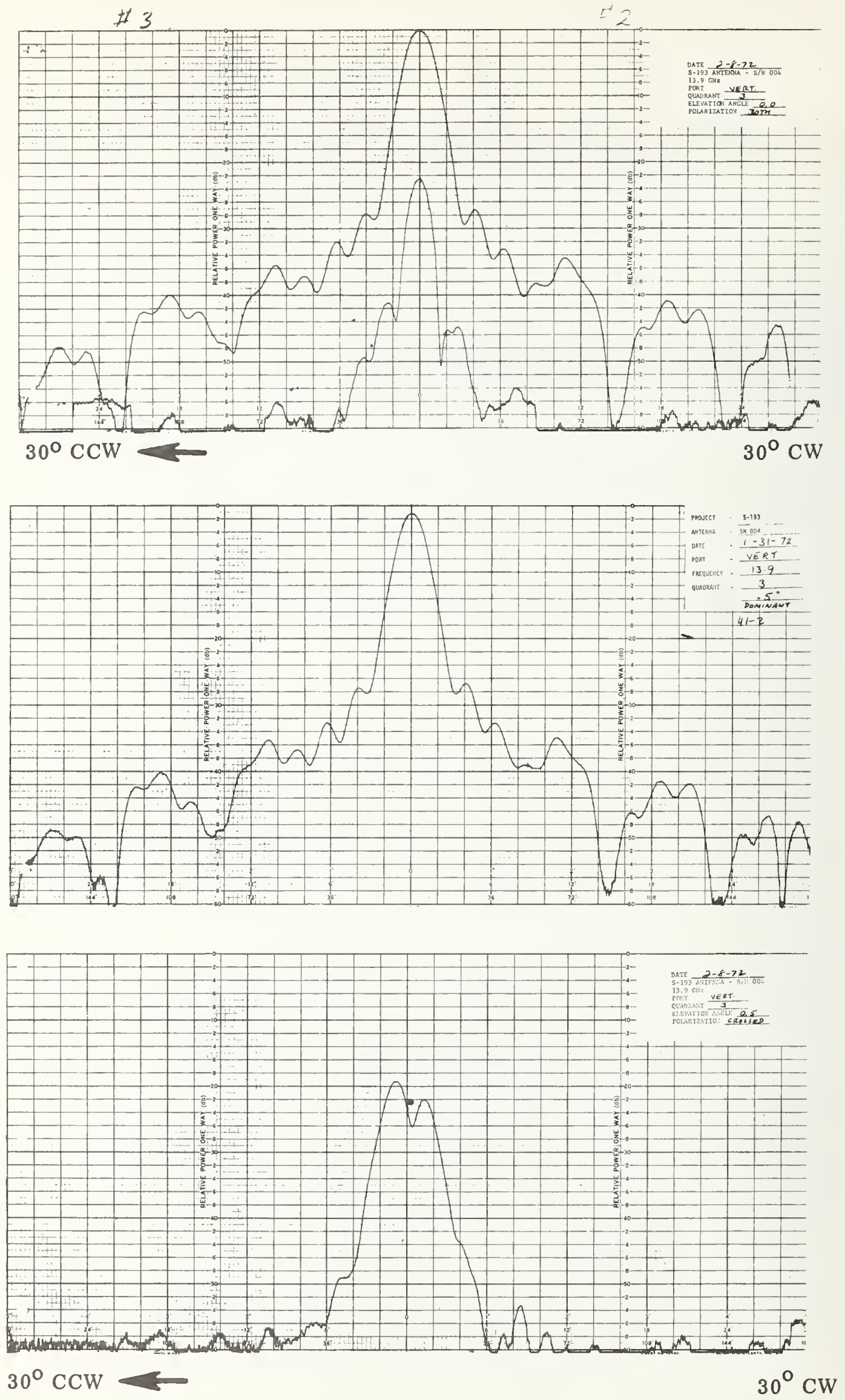

Figure 4-8. Pattern Cuts for the S-193 Antenna 

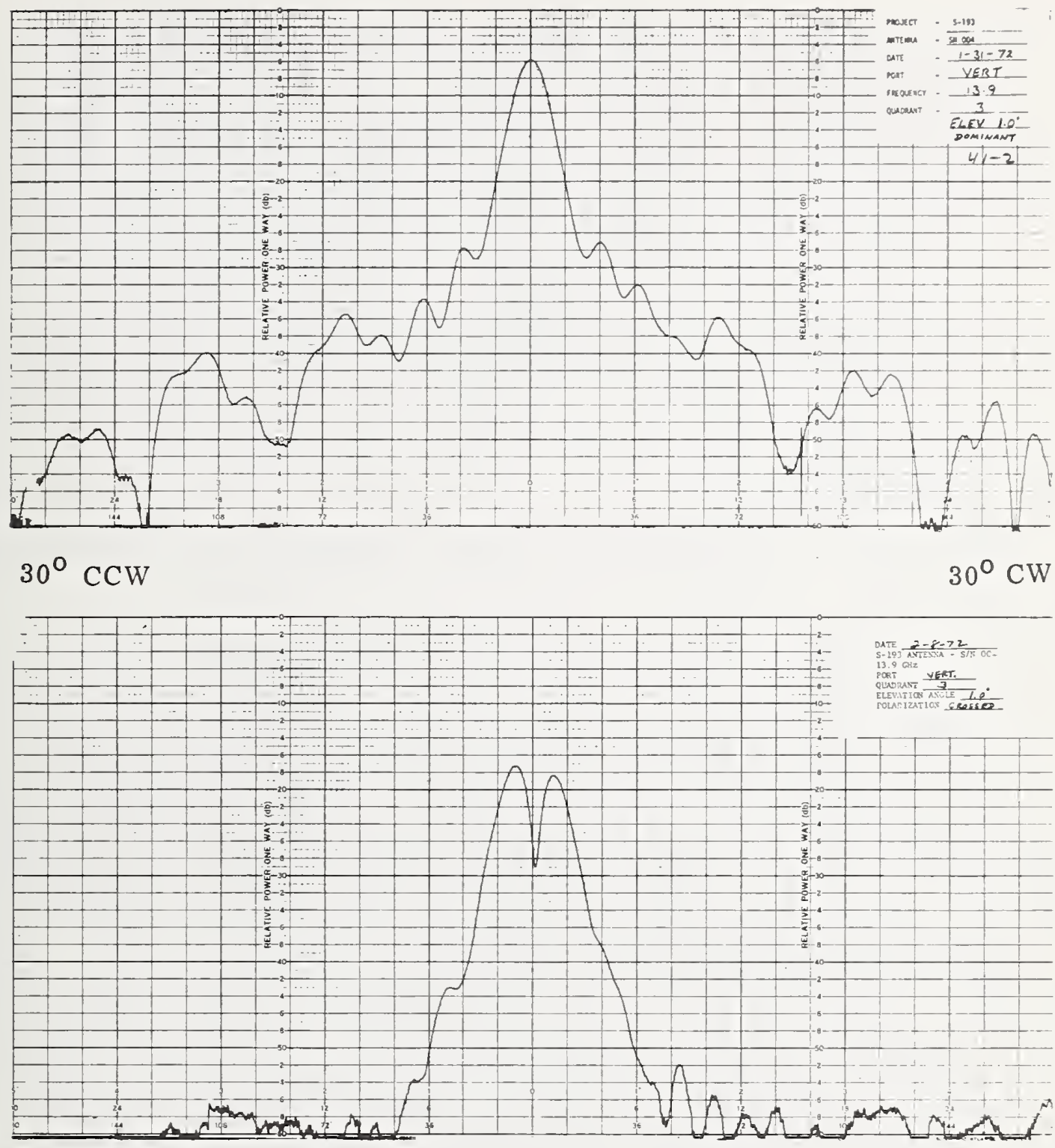

Figure 4-9. Pattern Cuts for the S-193 Antenna 
the ground. Because of the ground reflection problem, the antenna was reoriented on the rotator so that data could be obtained in quadrants 1 and/or 4 without pointing the antenna towards the ground. The reorientation consisted of rotating the test antenna and the source antenna about their $z$-axes by $90^{\circ}$ in a clockwise direction as viewed from the rear of the test antenna. In this orientation, azimuth cuts with positive elevation angles provided data in quadrants 2 and 1. Counter-clockwise rotation in azimuth and up in elevation would provide data from quadrant 2 in the "low reflection region" of the range.

While the reorientation did make low-reflection measurements possible in quadrant 2 and close to boresight in quadrant 1 , it also produced another small problem. The problem is, that due to the reorientation of the antenna on the mount, only principal plane cuts give strictly the same field components as were measured before reorientation along similar lines in "direction space." For instance, if the source antenna is linearly polarized in the $\bar{Y}$-direction for the initial antenna orientation, azimuth cuts at a constant elevation angle will yield patterns of the $\beta$ component along lines of constant $\beta$ (along straight lines in the direction space representation of figure 4-6). When the test antenna is reoriented as described above, and the source antenna is linearly polarized in the $\bar{X}$ direction, azimuth cuts at a constant elevation angle do not measure the $\beta$ component along lines of constant $\alpha$. The component measured is a combination of $\alpha$ and $\beta$, and the path in the direction space of figure 4-6 is curved. The total radiated power in quadrant 2 can be correctly determined from the sum of the powers in the two measured components but, strictly speaking, the measured major component powers in quadrants 2 and 3 should not be combined to give the power in the principal component. The same is true for the cross component power. 
Fortunately, for this antenna with its narrow beam, the effect due to reorientation is small over the significant part of the pattern. If we denote the measured components and rotator angles in the reoriented case by primes, $\left(E_{\beta}^{\prime}, E_{\alpha}^{\prime}\right.$, $\left.A Z^{\prime}, E L^{\prime}\right)$, and similar quantities in the original orientation without primes, we have

$$
E_{\beta}^{\prime}=-E_{\beta}\left(\cos ^{2} E L^{\prime}+\sin ^{2} E L^{\prime} \cos A Z^{\prime}\right)-E_{\alpha} \sin A Z \text { sin } E L
$$

for the case where

$$
E L^{\prime}=A Z \text { and } A Z^{\prime}=E L \text {. }
$$

When either $E^{\prime}=0$ or $A^{\prime}=0, E_{\beta}^{\prime}=E_{\beta}^{\prime}$; even at the edges of the significant part of the patterns where $E L=A Z \cong 12^{\circ}$,

$$
E_{\beta}^{\prime}=-0.9990 E_{\beta}+.04 E_{\alpha} \text {. }
$$

Since the difference between $E_{\beta}$ and $E_{\beta}^{\prime}$ is small, and only $E_{\beta}^{\prime}$ data is available in quadrant 2 , the following discussion will assume that they are equal. We will then estimate the error due to this assumption in the section on error analysis.

Analogue azimuth cut plots were taken in this new orientation at $0.1^{\circ}$ elevation increments between zero degrees and $6^{\circ}$ elevation, over the range from $-5^{\circ}$ to $+5^{\circ}$ azimuth. This was done for both polarizations, apparently to determine the symmetry between quadrants 2 and 1 . Samples of these plots are shown in figure 4-10. It is apparent from these and similar patterns at other elevation angles that there is significant non-symmetry here for both measured components. For the principal component, amplitudes are generally higher in quadrant 2 than in quadrant 1 . This is especially true for azimuth angles greater than $\pm 1^{\circ}$ where some differences are $5 \mathrm{~dB}$ or more. The null of the cross component is shifted off boresight into quadrant 2 , and the peak is also slightly lower in quadrant 2. Therefore there is a significant difference in the cross component powers in these two quadrants. 

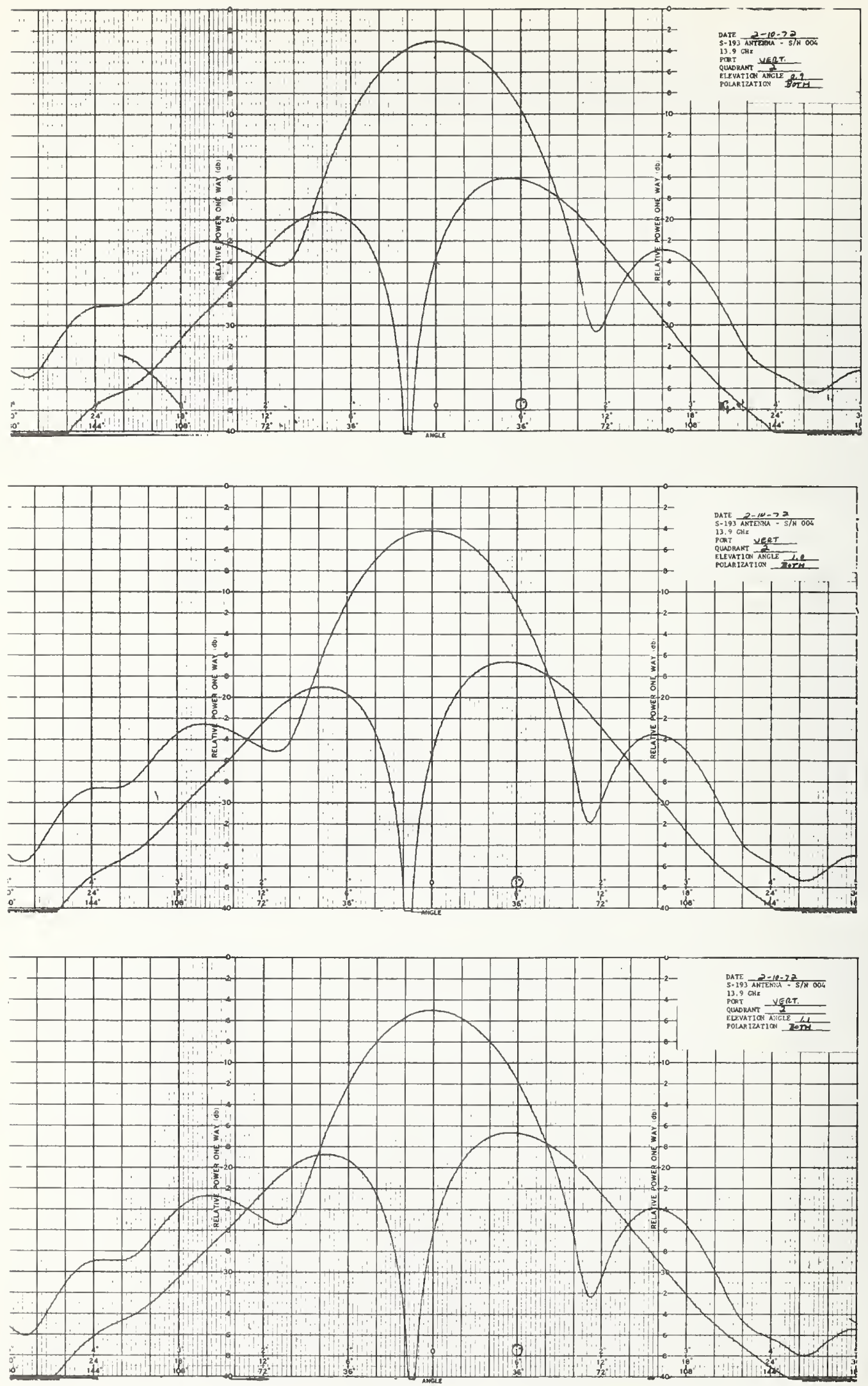

Figure 4-10. Principal and Cross Polarization Patterns for the Main Beam of the S-193 Antenna 
Again there are no patterns available between quadrants 3 and 4 . Apparently it was assumed that the symmetry properties there were the same as between 2 and 1 . Even with this assumption, the significant 2-1 non-symmetry would require that digital data be taken at least in quadrant 1 . However, digital data were not taken in quadrants 1 or 4 . Instead digital data were taken in quadrant 2. In view of the symmetry shown by figure 4-9, these data should give results quite similar to that obtained in quadrant 3 . But without any data in quadrants 1 or 4, 2-1 and 3-4 symmetry must be assumed to obtain total powers and beam efficiency, and this symmetry assumption is clearly not justified. It would have been much better to rotate the antenna on its axis by $180^{\circ}$, rather than $90^{\circ}$, to obtain actual $\alpha$ - and $\beta$-component data in quadrant 1 . Since this was not done, we can expect a significant error due to the as sumed symmetry. The magnitude of this error will be estimated in the error analysis section.

With the necessary 2-1 and 3-4 symmetry assumptions, the total power in each component is then given by twice the sum of the power for that component in quadrants 2 and 3. Thus,

$$
\mathrm{P}_{\mathrm{T} \beta}=2\left(\mathrm{P}_{2 \beta}+\mathrm{P}_{3 \beta}\right), \quad \mathrm{P}_{\mathrm{T} \alpha}=2\left(\mathrm{P}_{2 \alpha}+\mathrm{P}_{3 \alpha}\right)
$$

Since the limits of integration (or summation) for each partial power are different than for the total spherical integration implied in (4-18) and (4-19), these quantities are modified slightly to give

$$
P_{2 \beta}=\int_{0}^{\pi} \int_{0}^{\pi / 2} B_{1}(A Z, E L) \cos E L d E L d A Z .
$$

This integral was evaluated by the trapezoidal rule. Summation over the azimuth cuts yields

$$
\begin{aligned}
S_{2 \beta}\left(E L_{j}\right)=\cos E L_{j} \Delta A Z & \left(\frac{1}{2}\left[B_{1}\left(0, E L_{j}\right)+B_{1}\left(\pi, E L_{j}\right)\right]\right. \\
& \left.+\sum_{i=1}^{M-1} B_{1}\left(A Z_{i}, E L_{j}\right)\right) ;
\end{aligned}
$$


and further summation over the elevation increments gives

$$
P_{2 \beta} \cong \Delta E L\left(\frac{1}{2}\left[S_{2 \beta}(0)+S_{2 \beta}\left(\frac{\pi}{2}\right)\right]+\sum_{j=1}^{N-1} S_{2 \beta}\left(E L_{j}\right)\right) .
$$

In the above equations,

$$
\Delta \mathrm{AZ}=\frac{\pi}{\mathrm{M}}, \Delta \mathrm{EL}=\frac{\pi}{2 \mathrm{~N}}, \mathrm{AZ}_{i}=i \Delta \mathrm{AZ}, \mathrm{EL}_{j}=\mathrm{j} \Delta \mathrm{EL},
$$

and $\mathrm{S}_{2 \beta}\left(\mathrm{EL}_{j}\right)$ is the result of summing one azimuth cut as expressed in (4-27). In the measurements, both the azimuth and elevation measurement intervals varied over the area of measurement (see Fig. 4-7). This results in a further modification of equations (4-27) and (4-28) to account for the end points and $\triangle \mathrm{AZ}$ or $\triangle \mathrm{EL}$ of each interval. For instance the summation for each cut where there are two spacings is given by $S_{2 \beta}\left(E L_{j}\right)=\cos E L_{j}\left(\frac{\left[B\left(0, E L_{j}\right)+B\left(A Z_{M}, E L_{j}\right)\right] \Delta A Z_{1}}{2}\right.$

$$
\begin{aligned}
& +\frac{\left[B\left(A Z_{M}, E L_{j}\right)+B\left(\pi, E L_{j}\right)\right] \Delta A Z_{2}}{2} \\
& \left.+\Delta A Z_{1} \sum_{i=1}^{M-1} B\left(A Z_{i}, E L_{j}\right)+\Delta A Z_{2} \sum_{k=1}^{K-1} B\left(A Z_{M+k}, E L_{j}\right)\right),
\end{aligned}
$$

where

$$
\Delta \mathrm{AZ}_{1}=\frac{\mathrm{AZ} \mathrm{M}_{\mathrm{M}}}{\mathrm{M}}, \quad \Delta \mathrm{AZ} \mathrm{Z}_{2}=\frac{\pi-\mathrm{AZ} \mathrm{M}_{\mathrm{M}}}{\mathrm{K}} .
$$

A computer printout of the summation for the zero degree elevation cut in quadrant 2 for the horizontal port was provided by the contractor. The first page of this printout is shown in table 4-5. The power column is identical to $B_{1}\left(A_{i}, E L_{j}\right)$, and the "weighted P" column is equal to the power times $\triangle E L \cos E L$ for that cut. The $\triangle E L$ factor has therefore been included in the summation of (4-27) rather than in (4-28). Although the $\triangle E L$ for this run was 0.5 degrees, a value of 0.25 
Table 4.5 Computer Summary of Trapezoidal Rule Integration

ELEVATION ANGLE $=0.0$ DEGREES

FOR DOMINANT POLARIZATION

AZ IMUTH

ANGLE

DATA DECIBELS POWER

WEIGHTED P ACCUM

.083

$-1$

$-.07 \quad .98363$

.24591

.02049

.167

$-.07$

.98363

24591

.04098

.250

$-1$

$-.07$

.98363

.24591

.06148

.333

$-.29$

.93611

.23403

.08147

$-7$

.500

$-9$

$-.50$

.89088

.22272

.10051

.21549

.583

$-13$

$-.65$

.86195

.20172

.11876

.667

$-21$

$-.93$

.80638

.17677

.13615

.750

$-25$

.833

$-33$

.917

$-41$

1.000

$-55$

1.083

$-61$

1.167

$-70$

1. 250

1. 333

$-82$

1.417

$-99$

$-1.51$

.70707

.16547

.15192

$-1.79$

.66189

.14500

.16618

$-2.37 \quad .58002$

.12707

.17911

$-2.94 \quad .50827$

$-3.94$

.40340

.10085

.19045

$-4.37 \quad .36536$

.09134

.19995

$-5.02 \quad .31493$

$-5.88$

.25834

$-7.10$

.19513

$-7.81$

.16544

1.500

$-124$

$-8.89$

.12915

1.667

$-9.89$

.10251

1.750

$-138$

1.833

$-155$

$-11.11$

.07743

$-12.04$

.06247

1.917

$-168$

2.000

$-181$

2.083

$-196$

2.167

$-213$

2.250

$-227$

2.333

$-241$

2.417

$-258$

2.500

$-282$

2.583

- 294

2.667

$-312$

$-12.97$

.05041

$-14.05$

.03935

$-15.27$

.02972

$-16.27$

.02359

$-17.28$

.01872

$-18.49$

.01414

$-20.22$

.00952

$-21.08$

.00781

$-22.37$

.00580

$-23.87$

.00410

2.750

$-333$

$-25.81$

.00263

2.833

$-360$

2. 917

$-373$

3.000

$-393$

$-26.74$

.00212

$-28.17$

.00152

3. 083

$-421$

3. 167

$-445$

3. 250

$-457$

3. 333

$-472$

3. 417

$-486$

3.500

$-504$

3.583

$-515$

3. 667

$-523$

3.750

$-525$

3.833

$-515$

$-30.18$

.00096

$-31.90$

.00065

$-32.76$

.00053

$-33.84$

.00041

$-34.84 .00033$

.07873

.20796

.06458

.21504

.04878

.22101

.04136

. 22574

.03229

.22949

.02563

.23256

.01936

.23739

.01562

.23926

.01260

.24072

.24190

.00984

.24283

.00743

.24355

.00590

.24411

.00468

.24455

.00354

.24489

.00238

.24513

.00195

.00145

.24532

.24546

.00103

.24556

.00066

.24563

.00053

.24568

.00038

.24572

.00024

.24574

.00016

.24576

.00013

.24577

.00010

.24578

.00008

.24579

$-36.13 .00024$

.00006

.24580

$-36.92 .00020$

.00005

.24580

$-37.49 \quad .00018$

.00004

.24580

$-37.63 .00017$

.00004

.24581

$-36.92 .00020$

.00005

.24581

$-36.13 \quad .00024$

.00006

.24582

$-35.13 .00031$

.00008

.24582 
was used, which apparently is to account for the fact that this sum is for one of the end points in the sum over the elevation direction.

To obtain the values in the "accum" column, we multiply the weighted $P$ values by the appropriate $\triangle A Z$ and add to the preceding sum. This column represents the value of the summation as a function of the azimuth angle and points up one significant error in this summation. The error is that the end points of the summation interval have not been handled properly. Equation (4-30) states that the powers at the end points of each subinterval should be divided by two and then multiplied by the $\triangle A Z$ for that subinterval before being summed. It appears from the computer printout of table 4.5 that the first point of each cut, the $B\left(0, E L_{j}\right)$ point, has not been added in at all, and that the boundary point between subintervals, $B\left(30^{\circ}, \mathrm{EL}_{j}\right)$, and the end point, $\mathrm{B}\left(180, \mathrm{EL}_{\mathrm{j}}\right)$, have been added in as regular points of one subinterval. The engineer who performed the measurements and computations has indicated that the azimuth angle given in column 1 of table 4.5 is actually one increment ahead of the data. Therefore, the first row is actually for $A Z=0^{\circ}$, and instead of being left out, the first point has been added in as a regular point. Each cut summation is therefore in error by the amount $\delta_{\text {sum }}$ given by

$$
\begin{aligned}
\delta_{s u m}\left(E L_{j}\right)= & S^{\prime}\left(E L_{j}\right)-S\left(E L_{j}\right) \\
= & \left(\frac{B\left(0, E L_{j}\right) \Delta A Z_{j}}{2}+\frac{B\left(30, E L_{j}\right)\left(\Delta A Z_{2}-\Delta A Z_{1}\right)}{2}\right. \\
& \left.+\frac{B\left(\pi, E L_{j}\right) \Delta A Z_{2}}{2}\right) \Delta E L_{j} \cos E L_{j} \cdot
\end{aligned}
$$

Since $B\left(30, E L_{j}\right)$ and $B\left(\pi, E L_{j}\right)$ are both about $50 \mathrm{~dB}$ below the peak power, the only significant term in (4-32) is the one 
involving $B\left(0, E_{j}\right)$. This term is quite significant, however, since for the principal polarization $B\left(0, \mathrm{EL}_{j}\right)$ is the largest point of each cut. The error for each cut can be estimated by reading the value of $B\left(0, E L_{j}\right)$ from the analogue plots and computing the error from (4-32). The total error for that quadrant is the sum of the errors for each cut. For the case of quadrant 2 for the vertical port, the reported results of integration are

$$
\begin{aligned}
& \mathrm{P}_{2 \beta}=0.8373 \text { (degrees) }^{2} \\
& \mathrm{P}_{2 \alpha}=0.04397 \text { (degrees) }^{2}
\end{aligned}
$$

and the estimated error due to improper summation of the first points is

$$
\begin{aligned}
& \delta_{\text {sum } 2 \beta}=0.047{(\text { degrees })^{2}}^{2} \\
& \delta_{\text {sum } 2 \alpha}=0.0004(\text { degrees })^{2}
\end{aligned}
$$

More will be said about this error in the section on error analysis.

4.3.4 Beam Efficiency Calculations

By the usual definition, the beam efficiency is the ratio of the power in the main beam to the total radiated power,

$$
\varepsilon=\frac{\mathrm{P}_{\mathrm{m}}}{\mathrm{P}_{\mathrm{T}}},
$$

where the main beam is defined as the region from boresight to the first minimum of the principal polarization. The contractor has calculated $\mathrm{P}_{\mathrm{T}}$ as already described by trapezoidalrule integration of the measured patterns. They have not, however, used the correct boundary to calculate $\mathrm{P}_{\mathrm{m}}$. To obtain a main beam power, they have performed a second integration 
which employs a different set of angles than $\alpha$ and $\beta$ of the measurement system. The new angles are the usual spherical angles $\theta$ and $\phi$. The $z$-axis is the $\phi$-axis with $\phi$ measured from $x$ toward $y$ and $\theta$ measured from the $z$-axis as shown in figure 4-11. The measured data were then transformed from the $\alpha, \beta$ system to the $\theta, \phi$ system. A Gaussian quadrature integration was then used to compute the power as a function of the off-axis angle $\theta$, and it is this quantity which they have used to compute an approximate beam efficiency. However, this approach gives the correct beam efficiency only if the main beam boundary is at a constant angle from boresight. Figures 4-8 and 4-10 show that the minima are at about $2^{\circ}$ in one plane and are greater than $3^{\circ}$ in the other. Because of this, a single number has not been given as "the" beam effiency, but the contractor provided the graph in figure 4-12 which he termed the beam efficiency as a function of angle from boresight. For this they have used the ratio of power in the main polarization to total power in both polarizations.

For purposes of error analysis, we will use $2.5^{\circ}$ off axis as the "main beam" boundary to obtain a single number representing the beam efficiency, and then estimate the error due to the actual non-circular boundary.

\subsubsection{Error Analysis}

First, the results of the measurements and calculations are summarized for ease of reference. The measurements were made at a frequency of $13.9 \mathrm{GHz}$. Total powers were obtained from pattern measurements and integration in quadrants 2 and 3 and the symmetry assumptions previously discussed. For the vertical port the results of the pattern integrations are summarized in Table 4.6 . 


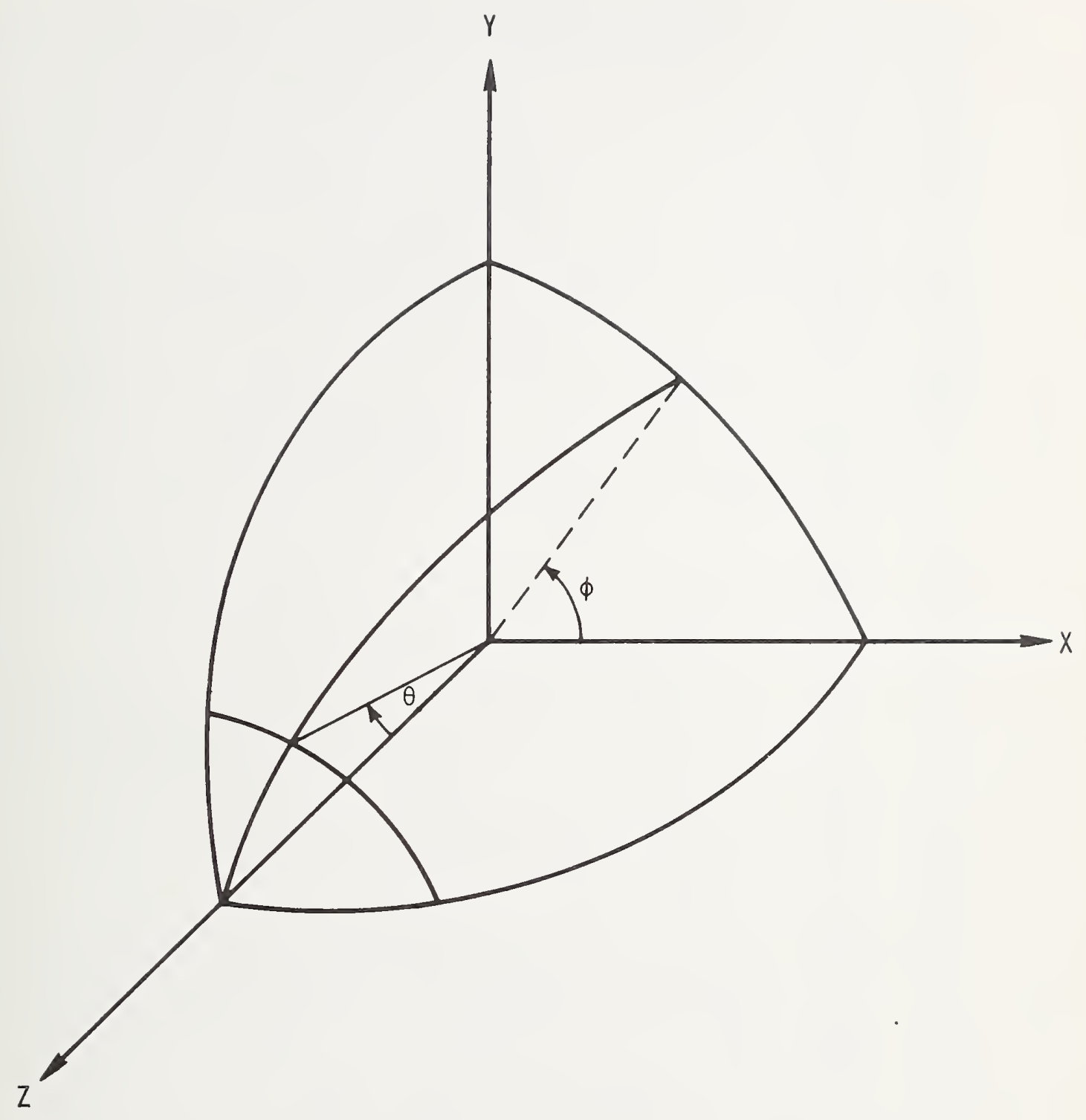

Figure 4-11. Spherical Coordinate System Used in the Analysis of the S-193 Antenna 


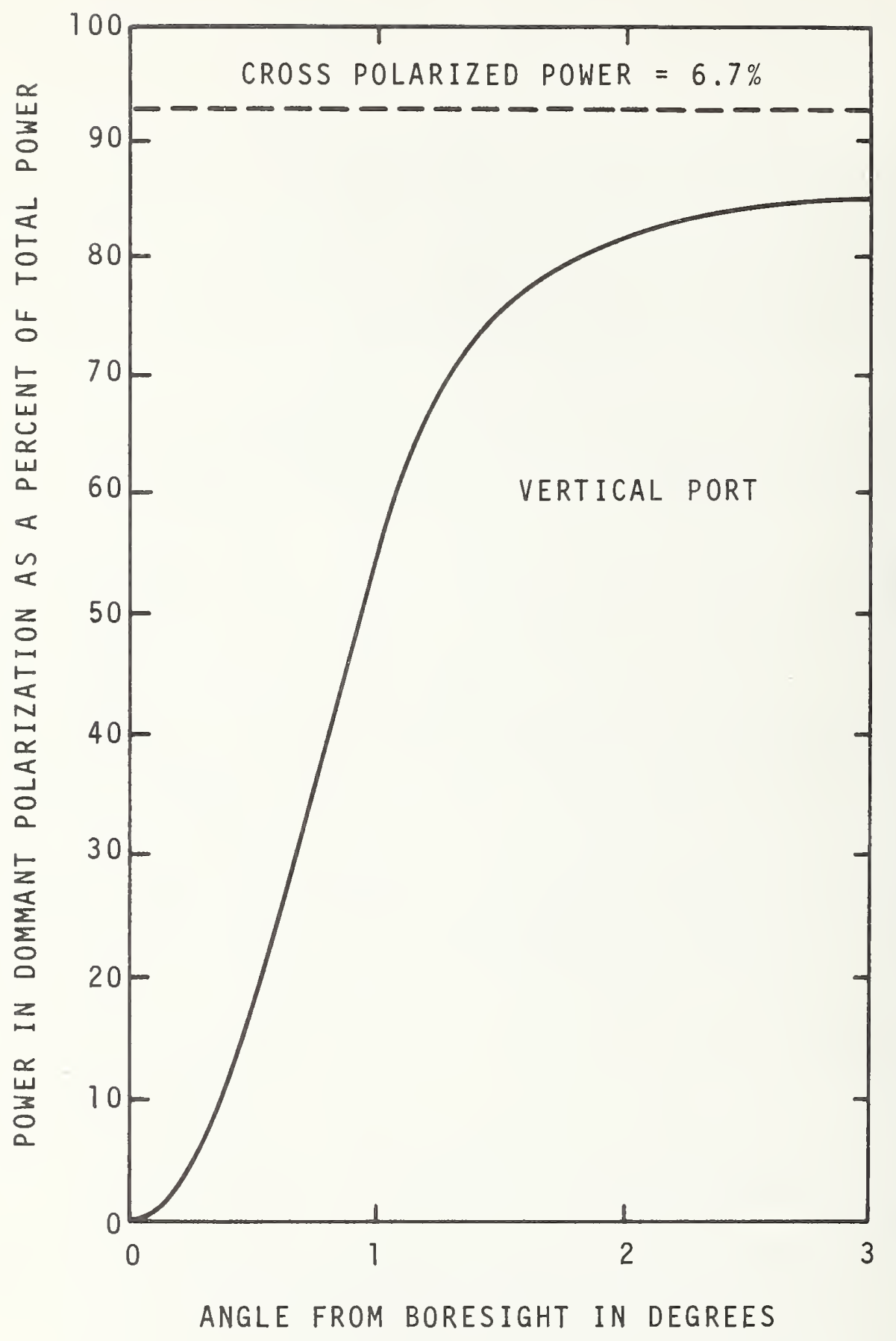

Figure 4-12. Contractor's "Beam Efficiency" vs Polar Angle (see page 94) 
Table 4.6 Results of Pattern Integration

\section{for Power in Each Quadrant}

$\frac{\text { Quadrant }}{2} \frac{\text { Component }}{\beta(\text { dominant })} \frac{\text { Power (degrees) }{ }^{2}}{.8373} \frac{\text { Power (Steradians) }}{2.550 \times 10^{-4}}$

$2 \alpha$ (cross)

.0440

$0.134 \times 10^{-4}$

$3 \quad \beta$

.8038

$2.448 \times 10^{-4}$

3

$\alpha$

.0756

$0.230 \times 10^{-4}$

$\mathrm{P}_{\mathrm{T}}=2\left(\mathrm{P}_{2 \beta}+\mathrm{P}_{3 \beta}+\mathrm{P}_{2 \alpha}+\mathrm{P}_{3 \alpha}\right)=10.724 \times 10^{-4}$ steradians

From figure 4-12 and estimates of the cross polarized power within $2.5^{\circ}$ of boresight,

$$
\mathrm{P}_{\mathrm{m}}=0.845 \mathrm{P}_{\mathrm{T}}+\mathrm{P}_{\mathrm{m} \alpha}=9.547 \times 10^{-4} \text { steradians. }
$$

The beam efficiency is

$$
\varepsilon_{\mathrm{T}}=\frac{\mathrm{P}_{\mathrm{m}}}{\mathrm{P}_{\mathrm{T}}}=89.0 \%
$$

for both polarizations, and

$$
\varepsilon_{\beta}=\frac{\mathrm{P}_{\mathrm{m} \beta}}{\mathrm{P}_{\mathrm{T}}}=84.5 \%
$$

for the principal polarization.

Using the computed total power and (4-6), the directivity obtained from pattern integration is

$$
\mathrm{D}_{\mathrm{P}}=\frac{4 \pi}{\mathrm{P}_{\mathrm{T}}}=40.69 \mathrm{~dB} .
$$

Using the measured gain of $41.1 \mathrm{~dB} \pm 0.37 \mathrm{~dB}$ and the efficiency of $-0.32 \mathrm{~dB}+.14 \mathrm{~dB}$, the directivity is

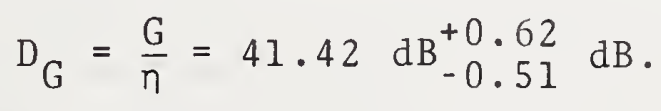

The errors which will be considered are due to pattern non-symmetry, non-linear polarization of source antenna, 
receiver system non-linearity, multipath interference, integration errors, incorrect main beam boundary, and reorientation of the antenna.

To estimate these errors we will use a slightly different approximate formula for the integral in (4-18) which employs the spherical angles $\theta$ and $\phi$ previously discussed. Let us denote the solid angle between the circular boundaries $\theta=a$ and $\theta=b$ by $\Omega_{a, b}$, which is given by

$$
\Omega_{a, b}=\int_{0}^{2 \pi} \int_{a}^{b} \sin \theta d \theta d \phi=2 \pi[\cos a-\cos b] \text {. }
$$

The total power for one component is then given by

$$
P_{B}=\sum \bar{B}_{a, b} \Omega_{a, b},
$$

where $\overline{\mathrm{B}}_{\mathrm{a}, \mathrm{b}}$ is the average value of the relative power pattern within the solid angle $\Omega_{a, b}$, and the summation is taken over the full $4 \pi$ steradians. The values of $\bar{B}_{a, b}$ can be estimated from the analogue patterns and from the Gaussian quadrature integration results. The results of this integration are shown in figure 4-12 which is a plot of the function

$$
\gamma(\theta) \equiv \frac{P_{\beta}(\theta)}{P_{T}} .
$$

By using the total power obtained from the first integration and the plot of the $\gamma(\theta)$ function, the average power/solid angle for one component is given by

$$
\bar{B}_{a, b}=\frac{(\gamma(b)-\gamma(a)) P_{T}}{\Omega_{a, b}} .
$$

For angles greater than $3^{\circ}$ and for the cross component, $\overline{\mathrm{B}}_{\mathrm{a}, \mathrm{b}}$ is estimated from the analogue plots.

Table 4.7 gives the pertinent data for the vertical port. With the values of $\bar{B}_{a, b}$ determined, we can now estimate how 
various sources of error could change the $\overline{\mathrm{B}}_{\mathrm{a}, \mathrm{b}}$ 's and therefore the calculation of beam efficiency and directivity.

Table 4.7. Estimates of Average Power Per Unit Solid Angle Within Solid Angle $\Omega_{a, b^{\circ}}$

Average

Polar Angles

Solid

Power Per in Degrees

Angle

Solid Angle

$\overline{\mathrm{B}} \mathrm{a}, \mathrm{b}$

Power in

\begin{tabular}{|c|c|c|c|c|c|c|}
\hline$a$ & b & $\Omega_{a, b}$ & $\overline{\mathrm{B}}_{\mathrm{a}, \mathrm{b}}$ & $\mathrm{dB}$ & $\mathrm{P}_{\mathrm{a}, \mathrm{b}} \times 10^{-4}$ & $\%$ of \\
\hline \multicolumn{7}{|c|}{ Principal Component } \\
\hline 0 & 1 & $9.5 \times \overline{10^{-4}}$ & .6434 & -1.91 & 6.113 & 57.0 \\
\hline 1 & 2 & $28.7 \times 10^{-4}$ & .0953 & -10.21 & 2.735 & 25.5 \\
\hline 2 & 3 & $47.8 \times 10^{-4}$ & .0079 & -21.0 & 0.375 & 3.5 \\
\hline 3 & 4 & $66.9 \times 10^{-4}$ & .0021 & -26.8 & 0.139 & 1.3 \\
\hline 4 & 5 & $86.0 \times 10^{-4}$ & .0010 & $-30 \cdot 0$ & 0.086 & 0.8 \\
\hline 5 & 6 & $105.1 \times 10^{-4}$ & .0005 & -32.9 & 0.054 & 0.5 \\
\hline 6 & 30 & 0.807 & $42.5 \times 10^{-6}$ & -43.7 & 0.343 & 3.2 \\
\hline 30 & 90 & 5.44 & $4.7 \times 10^{-6}$ & -53.2 & 0.257 & 2.4 \\
\hline 90 & 180 & 6.28 & $1.0 \times 10^{-6}$ & -60.0 & 0.064 & 0.6 \\
\hline
\end{tabular}

\section{Cross Component}

$\begin{array}{rrcccrr}0 & 2 & 38.3 \times 10^{-4} & .0081 & -20.9 & 0.311 & 2.9 \\ 2 & 4 & 114.8 \times 10^{-4} & .001 & -30 & 0.118 & 1.1 \\ 4 & 180 & 12.55 & 1 \times 10^{-6} & -60 & 0.129 & 1.2 \\ & & & & 10.724 & 100.0\end{array}$

\section{Pattern Non-Symmetry}

One of the largest sources of error is due to the significant non-symmetry between quadrants 2 and 1 or 3 and 4 . The magnitude of this error is estimated by a close examination of the analogue plots like those in figure 4-10. From 
these plots we can estimate the ratio of $\bar{B}_{a, b}$ in quadrant 2 to the same quantity in quadrant 1 , denoted by $\bar{B}_{a, b}^{\prime}$, and therefore estimate the resulting change in the partial and total powers from (4-35).

The results of this comparison are summarized in the table 4.8 .

Table 4.8. Change in Power Due to Non-Symmetry.

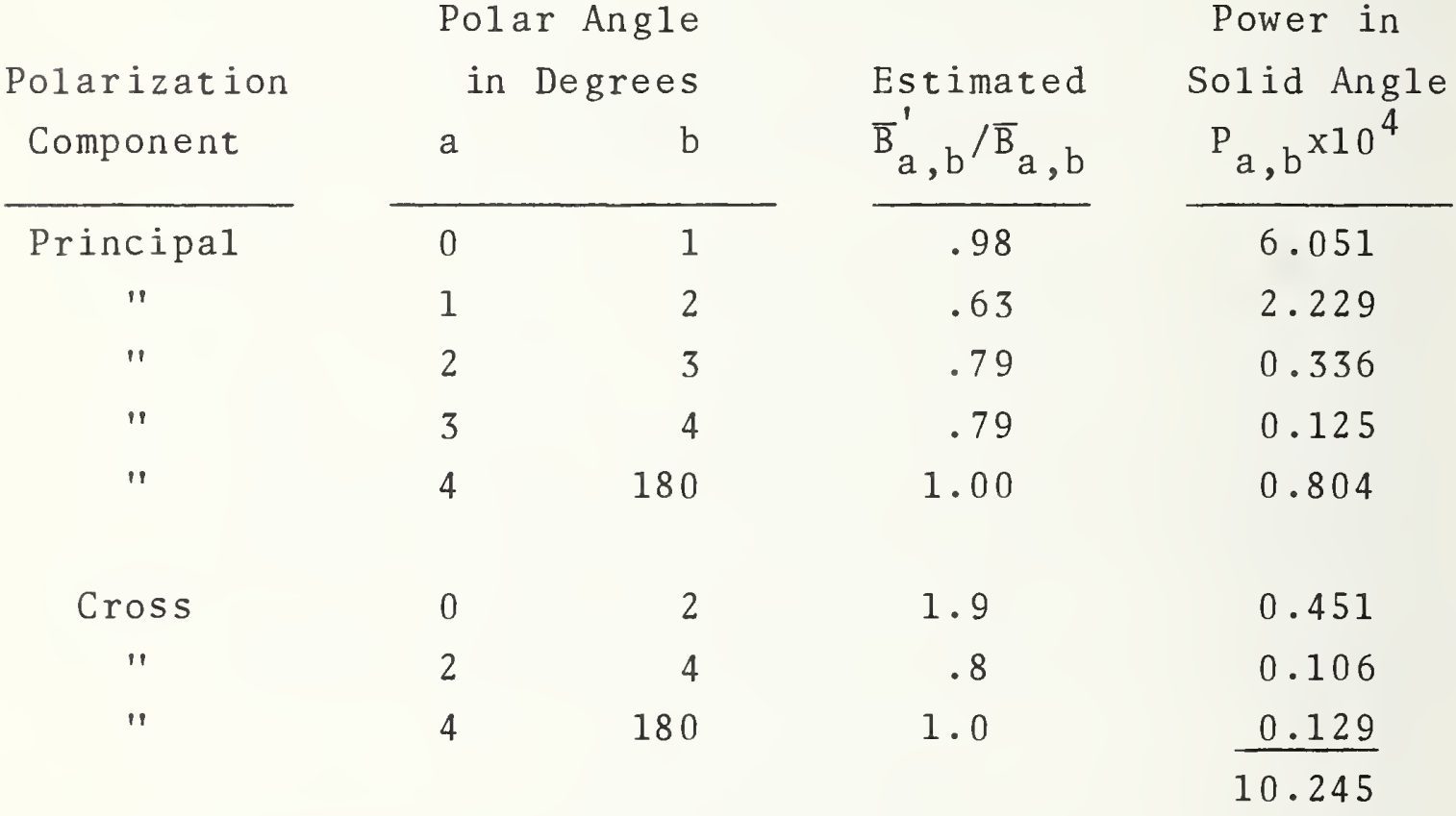

This indicates that the beam efficiency would be in error by $+2 \%$ and the directivity in error by $-0.2 \mathrm{~dB}$ due to the lack of symmetry in the 2-1 and 3-4 quadrant pairs.

There will be an additional error due to the non-symmetry between quadrants 1 and 4 . The apparent $1-4$ symmetry is probably about the same as the 2-3 symmetry, and therefore this error would be about equal to the measured difference between quadrants 2 and 3 . Since $\Delta \mathrm{P}_{\beta}$ was 0.102 and $\Delta \mathrm{P}_{\alpha}$ was 0.096 , the worst case estimate for the difference would be $\Delta \mathrm{P}_{\mathrm{T}}= \pm 0.198$. This would result in a change in $\varepsilon$ of $\pm 0.8 \%$, 
and a change in $\mathrm{D}$ of $\pm 0.08 \mathrm{~dB}$.

2. Non-Linear Polarization of Source Antenna

This error is due to the polarization factors in (4-16) and (4-17). Since the phases of the polarization ratios are not known, we will assume a worst case phase. The magnitude of $\rho_{\mathrm{S}}$ will be taken as 0.02 which corresponds to an axial ratio of $34 \mathrm{~dB}$. The magnitude of $\rho_{\mathrm{T}}$ will be estimated from the relative amplitudes of the principal and cross polarized patterns

$$
\left|\rho_{\mathrm{T}}(\alpha, \beta)\right|^{2}=\left|\frac{\mathrm{E}_{\alpha}(\alpha, \beta)}{\mathrm{E}_{\beta}(\alpha, \beta)}\right|^{2} \cong \frac{\mathrm{B}_{2}(-\alpha,-\beta)}{\mathrm{B}_{1}(-\alpha,-\beta)}
$$

From these estimates of $\rho_{\mathrm{T}}$ and $\rho_{\mathrm{S}}$, and the polarization factors $\left|1-\rho_{\mathrm{S}} \rho_{\mathrm{T}}(\alpha, \beta)\right|^{2}$ and $\left|1+\rho_{\mathrm{S}} / \rho_{\mathrm{T}}(\alpha, \beta)\right|^{2}$, the changes in $\bar{B}_{a, b}$ are estimated. These are tabulated with the error due to receiver non-linearity in Table 4.9.

3. Receiver Non-Linearity

If we assume a systematic error in the measured power patterns due to a receiver calibration error equal to $0.02 \mathrm{~dB} / \mathrm{dB}$, then the error in $\overline{\mathrm{B}}_{\mathrm{a}, \mathrm{b}}$ can easily be estimated. Although an estimate of the receiver linearity is not given in test reports, the error assumed here is reasonable for the type of receiver used. 
Table 4.9. Errors due to receiver non-1inearity and polarization mismatch.

\section{Principal Polarization}

Polar Angle Error in Receiver

Polarization

\begin{tabular}{|c|c|c|c|c|c|c|}
\hline in & $\begin{array}{c}\text { Degrees } \\
b\end{array}$ & $\begin{array}{c}\overline{\mathrm{B}}_{\mathrm{a}, \mathrm{b}} \\
\mathrm{dB}\end{array}$ & $\begin{array}{c}\text { Error } \\
\Delta \operatorname{Pxl} 0^{-4}\end{array}$ & & $\mid \rho_{\mathrm{T}}$ & \\
\hline 0 & 1 & 0.04 & 0.05 & & 0.1 & \\
\hline 1 & 2 & 0.2 & 0.10 & 0.3 & to & 10.0 \\
\hline 2 & 3 & 0.4 & 0.03 & 0.2 & to & 5.0 \\
\hline 3 & 4 & 0.5 & 0.01 & 0.1 & to & 2.0 \\
\hline 4 & 5 & 0.6 & 0.01 & & 1 & \\
\hline 5 & 6 & 0.7 & 0.007 & & 1 & \\
\hline 6 & 30 & 0.9 & 0.04 & & 1 & \\
\hline & 90 & 1.1 & 0.04 & & 1 & \\
\hline & 180 & 1.2 & 0.01 & & 1 & \\
\hline
\end{tabular}

Cross Polarization

\begin{tabular}{|c|c|c|c|c|c|c|}
\hline & & & $\Delta \mathrm{Px} 10^{-4}$ & $\left|\rho_{\mathrm{T}}\right|$ & $\left|1+\rho_{\mathrm{S}} / \rho_{\mathrm{T}}\right|^{2}$ & $\triangle \mathrm{Px} 10^{-4}$ \\
\hline 0 & 2 & 0.4 & 0.04 & 0.1 to 10 & 1.10 & 0.04 \\
\hline$?$ & 4 & 0.6 & 0.01 & 0.1 to 5.0 & 1.04 & 0.004 \\
\hline \multirow[t]{2}{*}{4} & 180 & 1.2 & 0.03 & 1 & & - \\
\hline & & TOTALS & \pm 0.38 & & \pm & 0.29 \\
\hline
\end{tabular}

The receiver error changes $\varepsilon$ by $\pm 1.3 \%$ and $D$ by $\pm 0.15 \mathrm{~dB}$, while the polarization factors change $\varepsilon$ by $\pm 0.03 \%$ and $D$ by $\pm 0.12 \mathrm{~dB}$. The error due to the polarization factor might be reduced if data were taken in all four quadrants. The phase of $\rho_{\mathrm{T}}(\alpha, \beta)$ would probably differ by $180^{\circ}$ between each quadrant due to the phase change of the cross component 
at its minimum point. This would make the polarization factor, $\left|1-\rho_{\mathrm{T}} \rho_{\mathrm{S}}\right|^{2}$, greater than unity in one quadrant, and less than unity in the other, thereby reducing the total effect of the polarization factor.

\section{Multipath Interference}

To estimate the effect of multipath, patterns were taken with the antenna in its original orientation and then rotated about its axis by $180^{\circ}$. Differences of 0.1 to $0.4 \mathrm{~dB}$ were observed over the main beam, and between 0.4 and $1.5 \mathrm{~dB}$ over the remainder of the pattern. With the small amount of information available, it is difficult to estimate the effect of these errors on the computed parameters. The character of the error will be similar to the receiver error, in that it should be small over the main beam and become larger at low signal levels. There will also be some periodic effect as the main and multipath signals go in and out of phase, which will reduce the total effect when integrated. A reasonable estimate is that the effect of multipath on the present measurements should be somewhat smaller than the error due to receiver non-linearity. We will use a $\Delta \varepsilon$ of $\pm 1.0 \%$ and $\Delta \mathrm{D}$ of $\pm 0.10 \mathrm{~dB}$.

\section{Integration Error}

The largest integration error is due to the improper summation of the initial point of each azimuth cut. It was previously estimated that this error resulted in the power in one quadrant being $0.144 \times 10^{-4}$ steradians too high. The total power will therefore be $0.576 \times 10^{-4}$ steradians too high, resulting in an error in $\mathrm{D}$ of $-0.24 \mathrm{~dB}$.

There is not enough information about the Gaussian quadrature integration to determine if a similar or additional error exists there for the beam efficiency calculation. If it was done correctly, and $\mathrm{P}_{\mathrm{m}}$ does not contain integration errors, then this above change in $\mathrm{P}_{\mathrm{T}}$ would result in an error in beam efficiency of $-5 \%$. 
Additional integration errors due to the size of the azimuth and elevation increments and the numerical summation should be on the order of $\pm 0.10 \mathrm{~dB}$ for $D$ and $\pm 1.0 \%$ for $\varepsilon$.

6. Incorrect Main Beam Boundary

This error results from using a circular boundary ( $\theta=$ constant) for the main beam when the actual boundary is not circular. This causes no error in the total power calculation, and therefore no error in directivity, but it does cause an error in the computed beam efficiency. It is apparent from figures 4-8 through 4-10 that the main beam boundary is between 1.8 and 3 degrees from boresight, and we have chosen 2.5 degrees to use as the approximate boundary. The resulting error in $\mathrm{P}_{\mathrm{m}}$ by this approximate boundary can be estimated by the change in the curve of figure 4-12 between 1.8 and 3 degrees. There is a change of $5.5 \%$ over this range. Since a circular boundary which contains the same power as the actual boundary is between 2.25 and 2.75 degrees, the error in $\varepsilon$ due to using the approximate boundary is $\pm 1 \%$. 7. Reorientation of Antenna

A description of how the reorientation of the antenna changed the measured components was given previously. This does not produce errors in the total power calculation or the total power in the main beam. It does produce a slight change in the power assigned to each component within the main beam. But since the main beam is so narrow, the change is very small and can be neglected.

\section{Error Summary}

The various sources of error are summarized in the following table. 
Table 4.10. Summary of Errors in

Beam Efficiency and Directivity.

Error in Calculated

Parameters

Source of Error

Non-Symme try

Polarization Factor

Receiver Non-Linearity

Multipath Signals

Integration Error

Main Beam Boundary

\begin{tabular}{|c|c|c|}
\hline & $\begin{array}{r}\text { Error } \\
\mathrm{P} \\
\end{array}$ & $\begin{array}{l}\text { In Calculated } \\
\text { ameters }\end{array}$ \\
\hline & $\Delta \varepsilon \%$ & $\Delta \mathrm{D}(\mathrm{dB})$ \\
\hline & $+2 \pm 0.13$ & $-0.20 \pm 0.13$ \\
\hline & \pm 0.03 & \pm 0.12 \\
\hline & \pm 1.3 & \pm 0.15 \\
\hline & \pm 1.0 & \pm 0.10 \\
\hline & $-5 \pm 2.0$ & $-0.24 \pm 0.18$ \\
\hline & \pm 1.0 & 0.0 \\
\hline TOTALS & $-3 \pm 2.8$ & $-0.44 \pm 0.3$ \\
\hline
\end{tabular}

The final results are then:

Total Beam Efficiency $=\varepsilon_{\mathrm{T}}=89.0 \%+3 \pm 2.8 \%$

Principal Beam Efficiency $=\varepsilon_{\beta}=84.5 \%+3 \pm 2.8 \%$

Directivity $=D=40.69 \mathrm{~dB}+0.44 \pm 0.3 \mathrm{~dB}$

\subsubsection{Recommendations}

The integration error could be reduced a great deal by correcting the summation of the end points. The program could be corrected and the data rerun, or the correction could be computed from the listing of the measured data and (4-32). If this is done, the results would be about

$$
\begin{aligned}
\varepsilon_{\mathrm{T}} & =94.0 \%-2 \pm 2.8 \% \\
\varepsilon_{B} & =89.5 \%-2 \pm 2.8 \% \\
D & =40.93 \mathrm{~dB}+0.2 \pm 0.3 \mathrm{~dB} .
\end{aligned}
$$


This would make the calculated directivity closer to the $41.42 \mathrm{~dB}$ obtained from gain and efficiency measurements. It would certainly be desirable to obtain data in quadrants 1 and/or 4 to reduce the error due to non-symmetry. It might be possible to read the data from the original plots like those of figures 4-8 to 4-10, which covered some of the area in quadrant 1 , and thereby obtain data out to at least $5^{\circ}$ in azimuth and $6^{\circ}$ in elevation. This would give data for the most significant non-symmetrical region of quadrant 1 , and would reduce the required symmetry assumptions and the associated error.

of course the best solution would be to actually make measurements in quadrants 1 and 4 over the full area and eliminate any symmetry assumptions.

If the symmetry assumptions could be eliminated, along with the integration errors, the results would be about

$$
\begin{aligned}
\varepsilon_{T} & =92.0 \% \pm 2.8 \% \\
\varepsilon_{B} & =87.5 \% \pm 2.8 \% \\
D & =41.13 \mathrm{~dB} \pm 0.3 \mathrm{~dB} .
\end{aligned}
$$

\subsubsection{Comparison of Gain, Efficiency and Directivity}

Due to the errors in the measured gain and in the directivity computed from pattern data, a more accurate efficiency cannot be obtained from the ratio of gain to directivity. However, the gain and efficiency can be used to compute a directivity which can be compared with the pattern directivity. This comparison indicates the consistency of measured data and error estimates. The directivity obtained from the gain/ efficiency ratio is $41.42 \mathrm{~dB}$, which is within the estimates of error for the directivity computed by pattern integration. The best agreement is with the value which has been corrected for non-symmetry and integration errors. This indicates that the corrections are fairly close. 
Another approach to improving the beam efficiency measurements might be to use the gain and efficiency results to compute the total power. If the gain results were improved as suggested in section 2.4 , this would yield a total power value with an error of about $\pm 0.7 \%$. Integration of existing or new pattern measurements over the main beam would then provide the main beam power, which would not be affected as much by sources of error as measurements over the complete angular range.

\subsection{S-193 Radiometer Receiver Error Analys is}

The radiometric portion of the S-193 Microwave Radiometer/ Scatterometer Altimeter System is analyzed in this section. This analysis includes: (I) a derivation of the noise output equation based on the radiometer model described in PIR No. U-1K00-S193-706, and in Specification No. SVS 7846, Revision C, 27 April 1972, p. 2-282; and (2) an analysis of this mode1 via the output equation for error in the value of antenna temperature determined. This analysis is not intended to supplant the contractor's analysis, but is performed as a check on the previous loss measurements and calculations. An estimate of the system resolution is given at the end of this section and should be considered as a limitation to the accuracy of the antenna temperature determined.

A simplified version of figure 2.7.3.2 of page 2-282 of the specifications is shown in Figure 4-13 for the mode in which the receiver is measuring the horizontal antenna output temperature TH. The symbols in the figure are defined as follows :

$\mathrm{TH}(\mathrm{TV})=$ antenna temperature as seen at input port $\mathrm{H}(\mathrm{V})$.

$\mathrm{TRF}=$ temperature of the "termination" on the isolated port circulator D.

$\mathrm{T} 1, \mathrm{~T} 2$ = noise temperatures of references 1 and 2 , respective1y. 


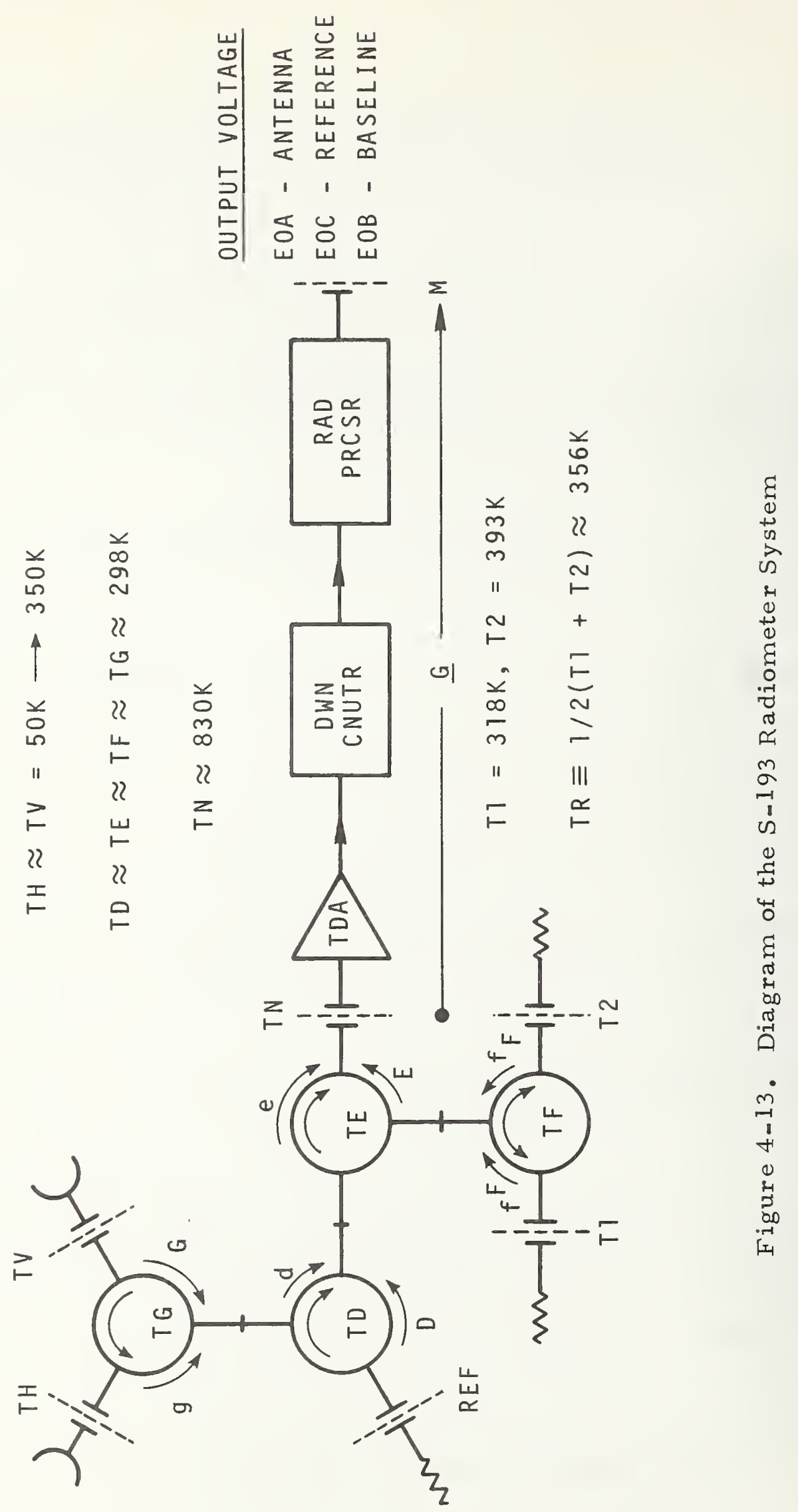


$\mathrm{TD}, \mathrm{TE}, \mathrm{TF}, \mathrm{TG}=$ temperature of the switching circulators $\mathrm{D}$, E, F, G respectively.

$\mathrm{TN}=$ effective input noise temperature of the TDA. $d, e, f, g$ represent available power ratios (see section 2 ) in the directions indicated for all equations through (4-43) and represent attenuations (see section 2) for all equations after (4-43) for circulators D, E, F, G respectively of the thru paths $\simeq 0.96$.

$D, E, F, G$ represent available power ratios in the directions indicated through equation (4-43), and thereafter attenuations of the isolation paths $\simeq 0.001$.

$\underline{G}=$ system gain as an available power ratio.

$M=$ mismatch factor at radiometer processor output which converts available power output to delivered or net power output.

EOA = output voltage when system is measuring antenna temperature TH.

$\mathrm{EOB}=$ zero offset or baseline voltage.

EOC = output voltage when system is measuring the difference noise temperature $\mathrm{T} 1$ - T2.

A number of reasonable assumptions which do not significantly affect the results have been made to ease the calculations. These assumptions are:

1. TN is the same for each mode of operation of the receiver (this should be a good assumption since the antenna and reference sources have comparable reflection coefficients).

2. The mismatch factor $M$ is independent of operational mode, allowing available powers (rather than net or delivered powers) to be used at the radiometer processor output port (rigorously true).

3. The measured values of $d, D, e, E$ etc., remain unchanged from laboratory measurement to final use. 
4. The gain G remains constant during the measurement.

5. When the circulators are switched, d and D, etc., change position. Although this assumption is not strictly valid, it is sufficiently true for the following analysis.

6. In the numerical estimates, $d=e=f=g, D=E=$ $\mathrm{F}=\mathrm{G}$, and $\mathrm{TD}=\mathrm{TE}=\mathrm{TF}=\mathrm{TG}$ are assumed.

Therefore, using the theory presented in section 2.1 of this report it can be seen that the output noise temperature $\mathrm{PH}$ at the TDA input when the system is in the horizontal mode is

$$
\begin{aligned}
\mathrm{PH}= & \mathrm{TH} \cdot \mathrm{g} \cdot \mathrm{d} \cdot \mathrm{e}+\mathrm{TV} \cdot \mathrm{G} \cdot \mathrm{d} \cdot \mathrm{e}+\mathrm{TG}(1-\mathrm{g}-\mathrm{G}) \mathrm{d} \cdot \mathrm{e} \\
& +\mathrm{TRF} \cdot \mathrm{D} \cdot \mathrm{e}+\mathrm{TD}(1-\mathrm{d}-\mathrm{D}) \mathrm{e}+\mathrm{TE}(1-\mathrm{e}-\mathrm{E}) \\
& +(\mathrm{T} 1 \cdot \mathrm{f}+\mathrm{T} 2 \cdot \mathrm{F}+\mathrm{T} 1 \cdot \mathrm{F}+\mathrm{T} 2 \cdot \mathrm{f}) \mathrm{E} / 2 \\
& +\mathrm{TF}(1-\mathrm{f}-\mathrm{F}) \mathrm{E}+\mathrm{TN}
\end{aligned}
$$

Equations can be written in a similar manner when the system is measuring the output noise temperatures P1, P2, and PR at the TDA resulting from viewing $\mathrm{T} 1$, $\mathrm{T} 2$, and $\mathrm{TR}$, respectively. In the antenna mode the output voltage EOA is given by

$$
\begin{aligned}
(E O A & -E O B) / G=P R-P H \\
& =T R \cdot f \cdot e+T F(1-f) e-T H \cdot g \cdot d \cdot e-T G(1-g) d \cdot e \\
& -T D \cdot(1-d) \cdot e \\
& +((T H-T G) \cdot g+(T V-T G) \cdot G+T G) \cdot d \cdot E \\
& +((T R F-T D) \cdot D+T D(1-d)) \cdot E-T E \cdot E \\
& +(T R-T F) \cdot F \cdot e \\
& -(T V-T G) \cdot G \cdot d \cdot e-(T R F-T D) \cdot D \cdot e \quad(4-40) \\
& -(T R-T F) \cdot(f+F) \cdot E-(T F-T E) \cdot E, \quad(4) \quad(4 R)
\end{aligned}
$$

which should be compared with equation (5) in the PIR.

In the calibration mode, the output voltage EOC is given by

$$
\begin{aligned}
\mathrm{K} \cdot(\mathrm{EOC}-\mathrm{EOB}) & =\mathrm{P} 1-\mathrm{P} 2 \\
& =(\mathrm{T} 1-\mathrm{T} 2) \mathrm{f}(\mathrm{e} \cdot \underline{\mathrm{G}})+(\mathrm{T} 2-\mathrm{T} 1) \mathrm{F}(\mathrm{e} \cdot \underline{\mathrm{G}}),
\end{aligned}
$$

which compares with equation (6) in the PIR, and $K$ is a gating constant described therein. $\underline{G}$ and e are grouped together 
because they appear naturally together in the analysis. Equation (4-41) is rearranged to obtain $e \underline{G}$ which is used in equation $(4-40)$.

The isolation terms (those terms involving one or more of the capital letters D, E, F, or G) in equations (4-40) and (4-41) are discarded in the contractor's analysis given in the PIR. By equating the small letters ( $d, e, f$, and $g$ ) to their approximate value of 0.96 , the capital letters to the approximate value of 0.001 , and the switch temperatures (TD, TE, TF, and TG) and TRF to their approximate value of $298 \mathrm{~K}$, the error introduced into $\mathrm{TH}$ (in kelvins) by discarding these isolation terms is

$$
9.3 \times 10^{-5}(\mathrm{TH}-\mathrm{TV})+(0.31,0.01) .
$$

The first term in (4-42) results from a cell (a cell on the earth's surface) polarization and should be negligible. The first and second values in the last term result from viewing a $50 \mathrm{~K}$ and $350 \mathrm{~K}$ cell, respectively.

Another source of error in determining TH from equations (4-40) and (4-41) comes from replacing the remaining available power ratios ( $d, e, f, g$ ) by attenuations (see section 2.1 for the definition of attenuation). An estimate of the size of this error can be obtained by examining the relationship between the available power ratio and the attenuation of the three port switching circulators. When this is done the resulting maximum errors in TH are, respectively, 0.02 and 0 , which are seen to be quite negligible.

After removing the isolation terms and replacing the remaining available power ratios by attenuations (from now on $d, e, f$, and $g$ refer to attenuations) the equations given then reduce to

$$
K(E O C-E O B)=f(T 1-T 2)(e \underline{G})
$$

and 


$$
\begin{aligned}
\mathrm{TH} & =f \cdot \mathrm{TR} / \mathrm{d} / \mathrm{g}+(1-f) \cdot \mathrm{TF} / \mathrm{d} / \mathrm{g} \\
& -(1-\mathrm{g}) \cdot \mathrm{TG} / \mathrm{g}-(1-\mathrm{d}) \cdot \mathrm{TD} / \mathrm{d} / \mathrm{g} \\
& +(\mathrm{EOB}-\mathrm{EOA}) / \mathrm{d} / \mathrm{g} /(\mathrm{e} \underline{\mathrm{G}}) .
\end{aligned}
$$

\begin{tabular}{|c|c|c|}
\hline Parameters & $\begin{array}{l}\text { Parameter } \\
\text { Uncertainty }\end{array}$ & $\begin{array}{l}\text { Resulting } \\
\text { Error in TH }\end{array}$ \\
\hline $\mathrm{TR}$ & $0.25 \mathrm{~K}$ & $0.26 \mathrm{~K}$ \\
\hline $\mathrm{TD}, \mathrm{TF}, \mathrm{TG}$ & $0.5 \mathrm{~K}$ & $0.02 \mathrm{~K}$ \\
\hline$E O B-E O A$ & 0.001 relative error & $(0.31,0.01) \mathrm{K}$ \\
\hline eG & 0.0026 & $(0.80,0.02) \mathrm{K}$ \\
\hline$d, f, g$ & 0.0023 & $(1.15,0.23) \mathrm{K}$ \\
\hline \multicolumn{3}{|c|}{$\begin{array}{l}\text { Probable Error } \\
\text { (Quadrature Sum) }\end{array}$} \\
\hline
\end{tabular}

After these equations are analyzed for errors in $\mathrm{TH}$ due to the various parameters, the following table of errors results:

\begin{tabular}{|c|c|c|c|}
\hline \multicolumn{2}{|c|}{ Parameter } & $\begin{array}{c}\text { Parameter } \\
\text { Uncertainty }\end{array}$ & $\begin{array}{l}\text { Resulting Uncer- } \\
\text { tainty in eG } \\
\end{array}$ \\
\hline $\mathrm{T} 1$ & $-\mathrm{T} 2$ & $0.039 \mathrm{~K}$ & 0.00067 \\
\hline & K & $9.8 \times 10^{-7}$ & $10^{-6}$ \\
\hline & $f$ & 0.0022 & 0.0023 \\
\hline \multirow[t]{2}{*}{ EOC } & $-E O B$ & 0.001 relative error & 0.001 \\
\hline & & Probable & 0.0026 \\
\hline
\end{tabular}

The parametric uncertainties leading to the uncertainty in eG quoted in the above table are tabulated below.

The analysis of the separate uncertainties in these two tables is straightforward, and since they do not indicate results significantly different from the contractor's values, the analysis is not included here.

The total probable error, DTH, in calculating TH from equations (4-44) and (4-45) is then the linear sum of the values given in $(4-42),(4-43)$, and $(4-46)$. This total is

$$
\begin{aligned}
\mathrm{DTH}= & 1.5 \mathrm{~K} \text { for a } 50 \mathrm{~K} \mathrm{cell} \\
& 0.3 \mathrm{~K} \text { for a } 350 \mathrm{~K} \mathrm{cell} .
\end{aligned}
$$


The theoretical one-sigma resolution, DT, of the S-193 radiometric receiver system (assuming no gain instability) is approximately [1]

$\mathrm{DT}=2 \cdot(\mathrm{TH} \cdot \mathrm{TH} / 2+\mathrm{TR} \cdot \mathrm{TR} / 2+\mathrm{TN} \cdot \mathrm{TN}+\mathrm{TH} \cdot \mathrm{TN}+\mathrm{TR} \cdot \mathrm{TN})^{\frac{1}{2}} /(\mathrm{B} \cdot \mathrm{T})^{\frac{1}{2}}$,

where $B$ is the limiting bandwidth (190 $\mathrm{MHz})$ of the system and $T$ is the integration time constant. The other symbols are defined in the figure. In this case $T N$ is referred to the switch input and is taken to be $1000 \mathrm{~K}$ instead of $830 \mathrm{~K}$ as shown in the figure. For a one-second time constant DT for both a $50 \mathrm{~K}$ and $350 \mathrm{~K}$ cell turns out to be approximately $0.2 \mathrm{~K}$. This value indicates that, assuming a Gaussian distributed output and no gain fluctuations, the output TH 1 ies within $\pm 0.2 \mathrm{~K}$ of the true averaged output with $68 \%$ probability. This 0.2 value is a lower limit to the system and it has been our experience that the actual value is at least a factor of 5 greater. Therefore, it seems reasonable to assume that the S-193 system resolution is around

$$
\mathrm{DT}=1 \mathrm{~K} \text {. }
$$

\section{$\underline{\text { References }}$}

[1] D.F. Wait, "Thermal Noise From a Passive Linear Multiport," IEEE Trans. Microwave Theory and Techniques, Vo1. MTT-16, No. 9, pp. 687-691, Sept. 1968.

[2] Glenn F. Engen, An Introduction to the Description and Evaluation of Microwave Systems Using Terminal Invariant Parameters, NBS Monograph 112 (Supt. Doc., Washington, D.C., Oct. 1969) pp. 14, 56.

[3] D.M. Kerns and R.W. Beatty, Basic Theory of Waveguide Junctions and Introductory Microwave Network Analysis (Pergamon Press, Oxford, 1967).

[4] M. Hamermesh, Group Theory and its Application to Physical Problems (Addison-Wesley Publ. Co., Inc., Reading, Mass., 1962). 
[5] D.M. Kerns, "Analys is of symmetrical waveguide junctions," J. Res. Nat. Bur. Stand. (U.S.), 46, No. 4, 267-282, (Apri1 1951).

[6] Theodore Moreno, Microwave Transmission Data (McGraw-Hil1, New York, 1948) p. 132 .

[7] Ibid., pp. 138-139. 


\begin{tabular}{|c|l|}
\hline $\begin{array}{c}\text { U.S. DEPT. OF COMM. } \\
\text { BIBLIOGRAPHIC DATA } \\
\text { SHEET }\end{array}$ & $\begin{array}{l}\text { 1. PUBLICATION OR REPORT NO. } \\
\text { NBSIR } \quad 73-314\end{array}$ \\
\hline
\end{tabular}

4. TITLE AND SUBTITLE

Evaluation of Antenna Loss and Beam Efficiency Measurements and Radiometer Calibrations for the S-193 and S-194 Skylab Systems

7. AUTHOR(S) A.C. Newell, P.F.Wacker, D.F. Wait W.C. Daywitt, R. C. Baird

9. PERFORMING ORGANIZATION NAME AND ADDRESS

NATIONAL BUREAU OF STANDARDS, Boulder Labs. DEPARTMENT OF COMMERCE

Boulder, Colorado 80302
12. Sponsoring Organization Name and Address

National Aeronautics and Space Administration

Manned Spacecraft Center

Facility and Laboratory Support Branch

Houston, Texas

2. Gov't Accession
No.

5

5. Publication Date

May 1973

6. Performing Organization Code

8. Performing Organization

10. Project/Task/Work Unit No.

\section{6}

11. Contract/Grant No. 13. Type of Report \& Period

NBS Interagency $R$ eport $5 / 1-12 / 311972$

14. Sponsoring Agency Code

15. SUPPLEMENTARY NOTES

16. ABSTRACT ( A 200-word or less factual summary of most significant information. If document includes a significant

bibliography or literature survey, mention it here.)

The program represents an effort to determine the most appropriate technigues for measuring the critical parameters of the radiometer-antenna systems used in the NASA Earth Resources and Skylab programs. For this first phase, the contractors' measurements on the S-193 and S-194 Skylab systems were studied in detail. The measurec quantities included antenna loss, antenna power gain, antenna patterns (which yield directivity and beam efficiency), and radiometric noise temperatures.

From the analysis of the contractors' data and auxiliary information, best values and their estimated errors were determined for each of the parameters. In addition, specific recommendations were made for easy upgrading of individual measurements, and the errors thus reduced were estimated. For some of the parameters, errors attainable with the current state-of-the-art were estimated.

In the measurements of antenna "loss", both contractors determined insertion loss rather than the ratio of output available power to input available power, required for radiometric determinations. For systems with significant reflections, these two quantities may be rather different, and the available power ratio should always be specified.

Antenna measurements made in an anechoic chamber showed surprisingly large wall reflections, suggesting that such chambers should be used for cross polarization measurements only with great caution.

17. KEY WORDS (Alphabetical order, separated by semicolons)

Antenna; Antenna Loss; Beam Efficiency; Calibration and Measurement;

Radiometer

18. AVAILABILITY STATEMENT

— UNLIMITED.

$x$ FOR OFFICIAL DISTRIBUTION. DO NOT RELEASE TO NTIS.

\begin{tabular}{|l|l|}
$\begin{array}{c}\text { 19. SECURITY CLASS } \\
\text { (THIS REPORT) }\end{array}$ & 21. NO. OF PAGES \\
UNCL ASSIF IED & \\
\hline $\begin{array}{l}\text { 20. SECUR ITY CLASS } \\
\text { (THIS PAGE) } \\
\text { UNCL ASSIFIED }\end{array}$ & 22. Price \\
\hline
\end{tabular}




